\title{
Extending homeomorphisms of the circle to quasiconformal homeomorphisms of the disk
}

\author{
DAVID EPSTEIN \\ VLADIMIR MARKOVIC
}

\begin{abstract}
We prove that it is not possible to extend, in a homomorphic fashion, each quasisymmetric homeomorphism of the circle to a quasiconformal homeomorphism of the disk.
\end{abstract}

30C62, 37E10, 37E30; 57M50, 57M60

\section{Contents}

Section 1: Introduction Statement of theorem, discussion of new methods, related problems, history

Section 1: Outline of the proof An overview of the proof

Section 3: Metrics, topological groups and completeness The Teichmüller metric, various applications of the Baire Category Theorem

Section 4: An important one-parameter group Strip analysis and the main a priori estimates of the paper

Section 5: Support of an extended map Statement of a key result, namely, if a quasisymmetric homeomorphism is fixed in an open interval then its extension to the disk fixes a neighbourhood of that interval; an outline of the proof of this result Section 6: The model and its properties A deeper look at strip analysis

Section 7: Completion of the proof of Theorem 5.1

Section 8: The dominion of an interval The group of quasisymmetric homeomorphisms supported on an interval extends in a controlled way to the disk

Section 9: The case $F\left(x_{0}\right) \neq\left\{x_{0}\right\} \quad$ A contradiction to strip analysis

Section 10: The case $F\left(x_{0}\right)=\left\{x_{0}\right\}$ The main case, where continuity is proved

Section 11: The affine group on an interval Conjugating into an interval affine maps and some other homeomorphisms of $\mathbb{R}$

Section 12: Continuous extensions Completion of the proof of Theorem 1.1

Section 13: Standard results A summary of known results, mostly about quasiconformal maps, but also including one topological result 


\section{Introduction}

Let $Q \mathcal{C}$ be the group of quasiconformal homeomorphisms of the closed unit disk $\overline{\mathbb{D}^{2}}$ in $\mathbb{C}$ to itself and let $Q \mathcal{S}$ be the group of quasisymmetric homeomorphisms. Restriction to the boundary circle gives a surjective homomorphism $q$ : $Q \mathcal{C} \rightarrow \mathcal{Q S}$.

Our main theorem is related to some important problems in surface theory, as described in this section,

1.1 Theorem (Stop Dreaming) There is no homomorphism $\mathcal{E}:$ QS $\rightarrow$ QC such that the composite

is the identity.

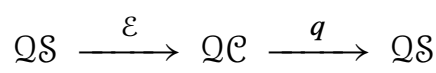

$\mathcal{E}$ stands for "Extend". Note that we make no continuity assumptions about $\mathcal{E}$.

The Dream Problem (named by Dennis Sullivan) was to construct the homomorphism whose non-existence is proved by our theorem. The name indicates the general belief that the existence of such a one-sided inverse is a fantasy and a delusion. It also indicates a delightful, dreamlike situation, where all kinds of wonderful results in surface theory are provable.

There are analogous problems for different classes of homeomorphisms, for example diffeomorphisms, where the solution is also negative, and homeomorphisms, where the solution is positive. At first contact, it may appear that homeomorphisms and diffeomorphisms are more natural objects than quasisymmetries. However, a homeomorphism of a compact Riemann surface of genus greater than one lifts to a quasi-isometry of its universal covering disk, and the boundary values of the lift define a quasisymmetry. For this reason, our investigations may be regarded as part of mainstream low-dimensional topology.

1.2 Methods The main new aspects of our proof are:

(1.2.1) We are able to use the Baire Category Theorem as a substitute for continuity of the homomorphism $\mathcal{E}$. This method may be applicable whenever one considers a not necessarily continuous homomorphism from a domain group with a complete left-invariant metric (or the structure of a locally compact topological group), to a range group that is the union in some natural way of a countable collection of subsets.

The use of the Baire Category Theorem as a substitute for continuity has been used previously by Epstein in [5] and, later, by Epstein and Thurston in [7], in a study of fibre bundles in a category theoretical context. 
(1.2.2) We undertake a study of not necessarily continuous one-parameter subgroups of quasiconformal homeomorphisms of the disk or strip, where the action on the boundary is of a specific nature. Surprisingly, it turns out that a priori estimates can be proved, as in Section 4.

In fact an important part of our analysis would go through for a single homeomorphism which is a quasi-isometry for the hyperbolic structure on the disk, provided the action on the boundary is of the right kind. (But we do not try to follow this direction in the current paper.) We construct geometric objects that separate the disk, based on the dynamics of the cyclic group generated by the homeomorphism, and use these to prove, for example, that there are no unexpected fixed points. This would fail without the quasi-isometry condition. We are able to gain geometric control on all iterates of our homeomorphism, even though the quasi-isometry constants of the iterates blow up. Since every quasiconformal homeomorphism is a quasi-isometry (see Theorem 13.6), the same results hold for a single quasiconformal homeomorphism.

The behaviour of our homeomorphism on the boundary has important features in common with the lifting to the universal covering disk of a pseudo-Anosov homeomorphism of a surface. Since any homeomorphism of a compact surface lifts to a quasi-isometry of the disk, our methods lead to new insights into the mapping class group of a compact surface. Indeed, basing his work on the ideas in this paper, and using some additional ingredients, Markovic [16] has proved, in the case of closed surfaces $S$ of genus 6 or more, that there is no group of homeomorphisms $G$ of $S$, such that the natural homomorphism of $G$ to the mapping class group is a surjective isomorphism. Markovic's student, Giblin, has proved the same result in genus 5 [11].

(1.2.3) Another central aspect of our work is a very carefully chosen conjugation of the action of the affine group acting on $\mathbb{R}$ in the usual way, so that, after conjugation, it acts on a finite interval. Outside the interval, we extend the action by the identity. Mather in [18] was, we believe, the first to investigate the conjugation of single diffeomorphisms to a finite interval. In [20], Plante showed how to make the affine group act with support in a closed halfline. Tsuboi in [22] showed how to conjugate the affine group with support in a finite interval. In our paper, we demand considerably more than is available from previous results, because we need to conjugate not only the affine group, but also earthquakes and the squaring map to quasisymmetric homeomorphisms. Neither the squaring map nor earthquakes are diffeomorphisms. Moreover, to enable our proof to go through, we need to be able to make the quasisymmetry constants of the conjugates of the affine transformations arbitrarily near those 
of the identity map. It seems that even slight changes in the formula for the conjugating diffeomorphism would spoil our proof, and we are fortunate to have been able to find the correct formula, and that it is so simple and explicit.

Here are two problems that would have been solved in the affirmative if the Dream Problem had a positive answer:

1.3 Problem Let $S$ be a compact oriented surface, and let $G$ be the group of orientation preserving homeomorphisms of $S$. Let $M$ be the mapping class group of $S$, that is, $G$ modulo isotopy, or, equivalently, $G$ modulo homotopy. Is the natural surjective homomorphism $G \rightarrow M$ split?

If the Dream Problem had a positive answer, one could argue as follows. If $S$ is a sphere or torus, then $G \rightarrow M$ is well-known to split. We therefore assume that $S$ is an oriented surface of genus at least two. Put a hyperbolic structure on $S$. The universal cover of $S$ is $\mathbb{H}^{2}$, the hyperbolic plane. The fundamental group of $S, \pi_{1}(S)$, acts on $\mathbb{H}^{2}$ by covering translations which are isometries of the hyperbolic structure. A homeomorphism of $S$ lifts to a homeomorphism of $\mathbb{H}^{2}$. The lifting is unique up to composition with a covering transformation.

Although the representative of an element $m \in M$ can be varied by an isotopy, the lifting of the isotopy does not move points on $\mathbb{S}^{1}=\partial \mathbb{U}^{2}$. (There is a fixed bound on the hyperbolic distance that the lifting of an isotopy can move any point.) Let $L$ be the group of all homeomorphisms of $\mathbb{S}^{1}$ which arise from lifting some representative of some element of $M$. Then $\pi_{1}(S)$, acting as a group of covering translations, is a normal subgroup of $L$, with quotient equal to $M$. Since each element $m \in M$ is represented by the corresponding Teichmüller map of $S$, we can choose a quasiconformal lifting to $\mathbb{H}^{2}$. It follows that $L$ consists of quasisymmetric homeomorphisms.

If the Dream Problem had a positive answer, such that each Möbius transformation of the circle is extended to a Möbius transformation of the disk, we would be able to extend elements of $L$ so that $L$ becomes a group of quasiconformal homeomorphisms of $\mathbb{H}^{2}$. Dividing by the covering translations, we would have a group of quasiconformal homeomorphisms of $S$ which is isomorphic to $M$. This would give the desired splitting of $G \rightarrow M$, and the image of the splitting would consist of quasiconformal homeomorphisms.

As already stated, Markovic has shown [16] that such a splitting does not exist if the genus is at least 6 .

1.4 Problem Suppose we have a group $G$ of quasisymmetric homeomorphisms of $\mathbb{S}^{1}$, and a constant $k>0$, such that each element $g \in G$ has Teichmüller distance 
$d_{\mathcal{T}}(g, \mathrm{Id})<k$. Is it then true that $G$ is conjugate by a quasisymmetric homeomorphism to a group of Möbius transformations?

A long chain of development led to the proof that such a group $G$ is conjugate by a homeomorphism to a group of Möbius transformations. We refer the reader to the works of Tukia [24], Hinkkanen [12], Gabai [8] and Casson and Jungreis [3], and also to the bibliographies in these papers. This work played a central role in the solution of Thurston's Geometrization Conjecture in the case of Seifert fibre bundles.

If a strong form of the Dream Problem were true, so that a bound on the quasisymmetric constant implies a bound on the quasiconformal extension, then the problem could be attacked by looking instead at a group of quasiconformal homeomorphisms of $\mathbb{D}^{2}$ with uniformly bounded dilatation. In dimension two, the problem was solved by Sullivan in [21] and by Tukia in [23] — they find a quasiconformal conjugacy. So the conjugacy by a homeomorphism of $\mathbb{S}^{1}$ could then be improved to a conjugacy by a quasisymmetric homeomorphism. This result was known in some cases, due to work of Hinkkanen in [13].

Although the Dream Problem is proved incorrect in this paper, and no such splitting exists, Markovic [17] has been able to make the improvement, proving the existence of a quasisymmetric homeomorphism that conjugates $G$ to a group of Möbius transformations.

1.5 Previous work Our study follows on a history of significant related results. In [2], Beurling and Ahlfors studied quasisymmetric homeomorphisms, giving in particular a formula for extending a quasisymmetric homeomorphism to a quasiconformal homeomorphism. This direction culminated in the frequently cited paper [4] by Douady and Earle. They gave a formula for a continuous map $\mathcal{E}_{\mathrm{DE}}$ : $Q \mathcal{S} \rightarrow \mathrm{QC}$. In fact the domain of their map consists of all homeomorphisms of the circle. The group $M$ of Möbius transformations preserving the unit disk is equal to the group of Möbius transformations of the unit circle preserving the orientation of the circle. The Douady-Earle extension has the property that it sends $M$, regarded as a group of quasisymmetric homeomorphisms of the unit circle, to $M$, regarded as a group of quasiconformal homeomorphisms of the unit disk, by the identity map. $\varepsilon_{\mathrm{DE}}$ is also left and right equivariant for composition by such transformations. It is not, however, a homomorphism between groups.

In [10], a clever argument by Ghys shows that there is no homomorphism $\mathcal{E}_{\mathrm{G}}$ from the group of diffeomorphisms of the circle to the group of diffeomorphisms of the disk, such that, for each diffeomorphism $f$ of the circle, $\mathcal{E}_{\mathrm{G}}(f)$ extends $f$.

If, in some context, an extension homomorphism does exist, then any such extension homomorphism can be changed using conjugation by a homeomorphism that is the 
identity on the boundary circle. We call such extension homomorphisms equivalent. One of the authors of this paper (Epstein) has shown that there are an uncountable number of inequivalent continuous extension homomorphisms from the group of homeomorphisms of the circle to the group of homeomorphisms of the unit disk. This work is not yet published.

1.6 The future In Problem 1.3 we showed that a positive solution to the Dream Problem would have solved the problem of realizing the mapping class group of a compact surface by a group of homeomorphisms. The techniques used in our negative solution can be used to provide a negative solution of the mapping class group problem. The problem remains open for closed surfaces of genus 2, 3, 4 and 5, and for punctured surfaces of any genus (with exceptions a sphere with 1,2,3 or 4 punctures and a torus with one puncture). Further development of these methods is likely to lead to a solution in most of the outstanding problems.

The closed surface of genus 2 seems not to be treatable by these means. The mapping class group for a surface of genus 2 is known to have somewhat different properties from the higher genus cases. For example the centre of the mapping class group for genus 2 is non-trivial, and the action of the group on Teichmüller space is not faithful.

This problem was solved in the affirmative by Kerckhoff in [14] for finite subgroups of the mapping class group, where a complex structure is proved to exist on the compact surface, together with an appropriate finite group of automorphisms of the complex structure. In [19, Chapter 4], Morita showed that the whole mapping class group cannot be realized as a group of diffeomorphisms, provided the genus is at least five.

Here is another problem of interest. Is it true that any extension homomorphism

$$
\mathcal{E}: \operatorname{Homeo}\left(\mathbb{S}^{1}\right) \rightarrow \operatorname{Homeo}\left(\overline{\mathbb{D}^{2}}\right)
$$

is continuous when both domain and range groups are provided with the compact-open topology, or, equivalently, the topology of uniform convergence. Each member of Epstein's uncountable collection of inequivalent extension homomorphisms is continuous.

In [10], Ghys also showed that no extension homomorphism exists in higher dimensions, in the case of diffeomorphisms. Analogously, one can define $2 S_{n-1}$ to be the group of all homeomorphisms of $\mathbb{S}^{n-1}$ obtained by restricting quasiconformal homeomorphisms of the unit disk $\overline{\mathbb{D}^{n}}$. Another problem is to show that there is no extension homomorphism $\mathcal{E}_{n}: \mathcal{Q S}_{n-1} \rightarrow \mathcal{Q}_{n}$. 


\section{Outline of the proof}

Here we give a highly simplified overview of our proof. We hope this will help readers to follow the complex logical path. Unfortunately, part of the path, which we originally hoped would turn out to be obstruction free, turns out to involve crawling through some thorny thickets. We will not mention the thickets in this overview, though they may be only too evident when we present the gory detail. Readers following closely should emerge relatively unscathed. Those striking out on their own should beware of easily overlooked pitfalls.

One way to use this overview is to flip back and forth between the overview and the detailed proof. We think this may be easier than confining oneself to either one or the other.

(2.1.1) In many situations where continuity is not assumed, the Baire Category Theorem can provide a partial substitute. The first application of the Baire Category Theorem is made in Proposition 3.6 and Proposition 3.9.

(2.1.2) In Corollary 3.10 and Corollary 3.11, we show that an arbitrary non-continuous homomorphism $\mathcal{E}: \mathcal{Q S} \rightarrow$ QC satisfying the hypotheses of Theorem 1.1 does in fact possess some important properties reminiscent of continuity-a result that we found quite surprising when we discovered it. This is one of the most useful technical results proved in this paper.

(2.1.3) The next task is to carry out what we call strip analysis. The essential feature is the use of quasiconformality to control the dynamics of certain subgroups of $2 \mathcal{C}$ on the interior of the unit disk, in terms of the behaviour on the boundary. This is possible, even though the quasiconformal constants of iterated maps will in general go to infinity. For this reason, we believe that this kind of approach might be of interest in other problems.

Let $S$ be the infinite horizontal open strip in $\mathbb{C}$, with lower edge the $x$-axis and height $\pi$, and let $\mathbb{U}^{2}$ be the open upper halfplane. We have the conformal isomorphisms exp: $S \rightarrow \mathbb{U}^{2}$ and the Cayley transform $\mathbb{U}^{2} \rightarrow \mathbb{D}^{2}$. We use these conformal isomorphisms to conjugate the homomorphism $\mathcal{E}: \mathcal{Q S} \rightarrow Q \mathcal{C}$ of Theorem 1.1 to a homomorphism $\mathcal{E}_{S}: \mathcal{Q S}(\partial S) \rightarrow \mathcal{Q C}(S)$ from the group of quasisymmetric homeomorphisms of the boundary $\partial S$ of the strip to the group $\mathrm{QC}(S)$ of quasiconformal homeomorphisms of the strip. There is an important one-parameter group $V: \mathbb{R}_{+} \rightarrow \mathcal{Q} \mathcal{C}(S)$, a homomorphism from the positive reals to the group of quasiconformal homeomorphisms of the strip. We define $V(t)(x, y)=(t x, y)$ if $x \geq 0$ and $V(t)(x, y)=(x, y)$ if $x \leq 0$. As a matter of notation, we will not use $V: \mathbb{R}_{+} \rightarrow \mathcal{Q C}(S)$ in the rest 
of the paper. But we will consistently use the notation $v: \mathbb{R}_{+} \rightarrow \mathcal{L S}(\partial S)$, for the restriction of $V$ to $\partial S$. We compare $V$ with the (not necessarily continuous) one-parameter group given by $t \mapsto \mathcal{E}_{S}(v(t) \in \mathcal{Q} \mathcal{C}(S)$. The two groups act identically on $\partial S$. On $S$, quasiconformality forces the two groups to act in a very similar way, even though the actions are not identical.

(2.1.4) We construct a family $\left\{\ell_{t}\right\}_{t>0}$ (see Definition 4.8) of subspaces of $S$ which provide an important tool for our investigation. For a given $t>0$, the subspace $\ell_{t}$ is connected, has compact closure and null interior. The subspaces fill up the righthand side of $S$, without gaps. Although these subspaces are not known to be curves, we think of them as providing a foliation of the righthand side of $S$. We have $\overline{\ell_{t}} \cap \partial S=\{t, t+i \pi\}$. An important feature of this family of subsets is that it is defined dynamically, using iterated mappings from the one-parameter subgroup $t \mapsto \mathcal{E}_{S}(V(t))$. We deduce an important equivariance principle: for each $s>0$ and each $t>0, \mathcal{E}_{S}(V(t) \mid \partial S)\left(\ell_{s}\right)=\ell_{s t}$.

Every additional fact that one can prove about the $\ell_{t}$ make the proof of the main result, Theorem 1.1, easier: a particularly important aspect is the control we gain over the shape of $\ell_{t}$ with respect to the hyperbolic metric on $S$ (see (4.16.2)).

(2.1.5) A key result (Theorem 5.1) is that $\bigcup_{t>0} \overline{\left\{\ell_{t}\right\}}$ is contained in the fixed point set of the group $\mathcal{E}(G)$, where $G$ is the subgroup of $2 \mathcal{S}(\partial S)$ of quasisymmetric homeomorphisms supported on $\partial S \cap\{x \leq 0\}$. This difficult result takes several sections to prove. We provide an overview of its proof at the end of Section 5 .

(2.1.6) Given an interval $[a, b] \subset \mathbb{S}^{1}$, we define $D(a, b)$ to be the fixed point set of the image under $\mathcal{E}$ of the group of all quasisymmetric homeomorphisms with support in the complement of $(a, b)$. We define $B(a, b)$ to be the frontier of $D(a, b)$. We define $F(x)$ to be the intersection of all the $D(a, b)$ with $x \in(a, b)$.

(2.1.7) The sets $D(a, b), B(a, b)$ or $F(x)$ are all defined canonically, and therefore, for each $f \in \mathcal{Q S}$,

$$
\begin{aligned}
\mathcal{E}(f)(D(a, b) & =D(f(a), f(b)), \\
\mathcal{E}(f)(B(a, b) & =B(f(a), f(b)) \text { and } \\
\mathcal{E}(f)(F(x)) & =F(f(x)) .
\end{aligned}
$$

(2.1.8) The proof separates into two cases: either $F(x)=\{x\}$ or $F(x) \neq\{x\}$. If $F(x) \neq\{x\}$, we obtain a contradiction to what we know about strip analysis, as outlined in (2.1.3). 
(2.1.9) If $F(x)=\{x\}$, then we can prove that $\mathcal{E}: \mathcal{Q S} \rightarrow$ QC is continuous in a certain non-standard sense (see Theorem 10.1). The proof uses the Riemann Mapping Theorem and (2.1.2) to prove the version of continuity required. This discussion requires a carefully controlled limiting argument.

(2.1.10) The next step involves hard analysis, mostly analysis for real-valued functions of real variables. We work in $\mathbb{U}^{2}$, the upper halfplane. The key idea here is the introduction of a very special diffeomorphism $\psi_{\delta}: \mathbb{R} \rightarrow(-1,1)$, given by an explicit formula. The formula is not easy to find. Moreover, the probable result of any change in the formula would be to make the necessary proofs extremely difficult, if not impossible, to carry out convincingly. Using this explicit diffeomorphism, we are able to conjugate many quasisymmetric homeomorphisms of $\mathbb{R}$ to convert them into quasisymmetric homeomorphisms with compact support. In particular, all affine homeomorphisms of $\mathbb{R}$ can be so conjugated. The same applies to earthquakes- these are homeomorphisms $R_{a}: \mathbb{R} \rightarrow \mathbb{R}$, with $a>0$, defined by $R_{a}(t)=a t$ if $t \geq 0$ and by $R_{a}(t)=t$ if $t \leq 0$. It also applies to the squaring map $S: \mathbb{R} \rightarrow \mathbb{R}$ given by $S(t)=t^{2}$ if $t \geq 0$ and by $S(t)=-t^{2}$ if $t \leq 0$.

(2.1.11) By changing scale, and using other tricks, we are able to make the conjugates of the affine maps arbitrarily near the identity in terms of quasisymmetric constants. Using the non-standard version of continuity of $\mathcal{E}$ referred to in (2.1.9), we deduce that, after applying $\mathcal{E}$ to the conjugates of affine maps, we obtain quasiconformal homeomorphisms with bounded dilatation.

(2.1.12) An important result, due independently to Sullivan in [21] and to Tukia in [23], states that a group of quasiconformal homeomorphisms of the disk, with uniformly bounded dilatations, is conjugate to a group of Möbius transformations. A limiting process applied to the results of (2.1.11), together with the Sullivan-Tukia result just stated, allows us to change $\mathcal{E}$ to a related homomorphism $\widehat{\varepsilon}$ having the following property. Let $a>0$ and $b \in \mathbb{R}$. Let $F_{a, b}: \mathbb{C} \rightarrow \mathbb{C}$ be defined by $F(z)=a z+b$. Then $\widehat{\mathcal{E}}\left(F_{a, b} \mid \mathbb{R}\right)=F_{a, b} \mid \overline{\mathbb{U}^{2}}$.

(2.1.13) In Theorem 12.12 we compute and give a precise formula for $\widehat{\mathcal{E}}\left(R_{a}\right)$. This step depends crucially on Theorem 5.1, referred to in (2.1.5). We are then able to compute a precise formula for $\widehat{\mathcal{E}}(S)$, where $S: \mathbb{R} \rightarrow \mathbb{R}$ is the squaring map, given by $S(x)=x^{2}$ if $x \geq 0$ and by $S(x)=-x^{2}$ if $x \leq 0$.

(2.1.14) The precise formula for $\widehat{\mathcal{E}}(S)$ shows that it is not quasiconformal. This contradiction completes the proof of Theorem 1.1. 


\section{Metrics, topological groups and completeness}

Let $u$ be a quasiconformal homeomorphism of an open subset of the plane. We denote by $K(u)$ the (almost everywhere or $L^{\infty}$ ) supremum of the pointwise dilatations of $u$.

3.1 Definition Given two quasiconformal homeomorphisms, $f$ and $g$ of the open unit disk $\mathbb{D}^{2}$ onto itself, we define the Teichmüller metric $d_{\mathcal{T}}(f, g)=\log \left(K\left(f^{-1} g\right)\right)$. This definition is due to Ahlfors (see [1]), who invented the concept.

Quasiconformal homeomorphisms that differ from each other by right translation by a Möbius transformation are at zero Teichmüller distance apart. So this is a pseudometric on $\mathcal{Q}$, rather than a metric, but we will nonetheless call it a metric. The Teichmüller metric is invariant under left composition with a quasiconformal homeomorphism.

In [1, Chapter 1], Ahlfors shows that the Teichmüller distance between two quasiconformal homeomorphisms $f$ and $g$ of $\mathbb{D}^{2}$ can be interpreted as a hyperbolic distance. One takes the Beltrami differentials $\mu_{f}$ and $\mu_{g}$ of $f$ and $g$. These are $L^{\infty}$ functions $\mathbb{D}^{2} \rightarrow \mathbb{D}^{2} \subset \mathbb{C}$. If $\mathbb{D}^{2}$ is given the Poincaré metric, then it can be regarded as the hyperbolic plane and we can take the uniform (in the $L^{\infty}$ sense) distance between the two functions $\mu_{f}$ and $\mu_{g}$. We obtain precisely the Teichmüller distance.

Given three points of the boundary circle, we can take the subgroup $\mathcal{Q C}_{3}$ of $Q \mathcal{C}$ consisting of quasiconformal homeomorphisms that fix each of the three points. The Teichmüller metric is a true metric, when restricted to such a subgroup.

3.2 Definition We can also define the Teichmüller (pseudo)metric on QS. This metric is obtained by varying over all extensions to the unit disk, and minimizing the Teichmüller distance on the disk.

Again the Teichmüller metric is a left invariant pseudometric on $\mathcal{Q} \mathcal{S}$, but not a metric. When we restrict to a subgroup consisting of quasisymmetric homeomorphisms that fix the same three points, we obtain a true metric.

The following result is stated by Gardiner and Sullivan in [9].

3.3 Lemma Let $\mathcal{Q}_{3}$ be the group of quasiconformal homeomorphisms of the disk that fix $1,-1$ and $-i$. Then $\mathcal{Q C}_{3}$ is not a topological group with respect to the Teichmüller metric. The same applies to $\mathcal{Q S}_{3}$, the group of quasisymmetric homeomorphisms of the circle that fix $1,-1$ and $-i$. Although left translation is an isometry, there is, for each of these groups, an element $f$ such that right translation by $f$ is not continuous at the identity element. 
Proof We will work on the infinite horizontal strip $S$ of height 1 with lower edge the $x$-axis, restricting attention, for the most part, to quasisymmetric or quasiconformal homeomorphisms that are the identity for $x \leq 0$. Let $g_{n}: S \rightarrow S$ be defined by $g_{n}(x, y)=(x, y)$ for $x \leq 0$ and $g_{n}(x, y)=\left(\frac{(2 n+1) x}{2 n}, y\right)$ for $x>0$. Then $d_{\mathcal{T}}\left(g_{n}, \mathrm{Id}\right)=$ $\log \left(\frac{2 n+1}{2 n}\right)$ which converges to zero as $n$ tends to infinity. Let $H_{n}$ be the halfdisk with centre $(n, 0)$ and with radius $1 / n$. We choose $f: S \rightarrow S$ so that $f\left(H_{n}\right)=H_{n}$, so that $f$ is supported on $\bigcup_{n>3} H_{n}$, and so that, for each $n$, the dilatation $K\left(f \mid H_{n}\right)=2$. We compute $d_{\mathcal{T}}\left(f, g_{n} f\right)=d_{\mathcal{T}}\left(\mathrm{Id}, f^{-1} g_{n} f\right)$. Now $f^{-1} g_{n} f\left|H_{n}=g_{n} f\right| H_{n}$ and

$$
2=K\left(f \mid H_{n}\right) \leq K\left(g_{n}^{-1}\right) . K\left(g_{n} f \mid H_{n}\right) \leq \frac{2 n+1}{2 n} . K\left(g_{n} f \mid H_{n}\right) .
$$

Therefore

$$
K\left(f^{-1} g_{n} f\right) \geq K\left(f^{-1} g_{n} f \mid H_{n}\right)=K\left(g_{n} f \mid H_{n}\right) \geq \frac{4 n}{2 n+1}>1.5
$$

and so $d_{\mathcal{T}}\left(f^{-1} g_{n} f\right.$, Id $)>\log (1.5)$. This deals with Lemma 3.3 in the case of quasiconformal homeomorphisms.

To prove the result for quasisymmetric homeomorphisms, we use $g_{n} \mid \partial S$ as above, and a quasisymmetric homeomorphism $h: \mathbb{S}^{1} \rightarrow \mathbb{S}^{1}$ with support in $\bigcup_{n>3} \overline{H_{n}} \cap \partial S$ and such that $h\left(\overline{H_{n}} \cap \mathbb{S}^{1}\right)=\overline{H_{n}} \cap \mathbb{S}^{1}$. We further choose $h$ so that, for any extension $f_{n}: \bar{S} \rightarrow \bar{S}$ of $h \mid H_{n} \cap \partial S, K\left(f_{n}\right) \geq 2$. This will be true if, for example, $h$ is the identity immediately to the left of $(n, 0)$ on the $x$-axis, and expands by a suitable factor immediately to the right of $(n, 0)$. We have inequalities that are analogous to those of the previous paragraph, and these show that $d_{\mathcal{T}}\left(h^{-1} \circ\left(g_{n} \mid \mathbb{S}^{1}\right) \circ h, \mathrm{Id}\right)>\log (1.5)$, giving a contradiction as before.

3.4 Lemma $\mathcal{Q C}_{3}$ and $\mathcal{Q S}_{3}$ are complete with respect to the Teichmüller metric.

Proof This is standard. The proof goes as follows. Let $\left(g_{n}\right)_{n \in \mathbb{N}}$ be a Cauchy sequence in $\mathcal{L C}_{3}$. We need only prove that some subsequence $\mathcal{T}$-converges. The sequence has uniformly bounded dilatation. By Theorem 13.3, if we go to a subsequence, we may assume without loss of generality that our sequence converges uniformly on compact subsets. A crucial point is that it cannot converge to a point, because three points on the boundary are fixed. So the sequence converges to a quasiconformal homeomorphism, $g$, whose dilatation has the same bound.

Given $\epsilon>0$, there is an $N>0$, such that, for $n, m \geq N, d_{\mathcal{T}}\left(g_{n}, g_{m}\right) \leq \epsilon$. Letting $n$ tend to infinity, we find that $d_{\mathcal{T}}\left(g, g_{m}\right) \leq \epsilon$. 
In addition to the Teichmüller metric, we use the uniform metric $d_{u}$ on $Q \mathcal{C}$ and $Q \mathcal{S}$. Note that the uniform metric is right invariant and not left invariant. With respect to the uniform metric, $Q \mathcal{C}$ and $\mathcal{Q S}$ are topological groups, but are not complete. With respect to $d_{\mathcal{T}}, \mathcal{Q C}_{3}$ and $\mathcal{Q S}_{3}$ are complete, but not topological groups-see Lemma 3.3. The following lemma is standard and we omit the proof-see Section 13.

3.5 Lemma The identity maps $\left(\mathcal{Q C}_{3}, d_{\mathcal{T}}\right) \rightarrow\left(\mathcal{Q C}_{3}, d_{u}\right)$ and $\left(\mathcal{Q S}_{3}, d_{\mathcal{T}}\right) \rightarrow\left(\mathcal{Q S}_{3}, d_{u}\right)$ are continuous.

The following result is a consequence of the Baire Category Theorem that is crucial for our proof.

3.6 Proposition Let $\mathcal{E}: \mathcal{Q S} \rightarrow$ QC be the fixed homomorphism of Theorem 1.1 (that we are trying to show does not exist). Let $G \subset Q S_{3}$ be any subgroup that is closed with respect to the Teichmüller metric. Then there is an $\epsilon_{0}>0$, a $k_{0}>1$ and a dense subset $Q$ of the $d_{\mathcal{T}}-\epsilon_{0}$-ball centred at the identity in $Q \mathcal{S}$, such that, if $g \in Q$, then $d_{\mathcal{T}}(\mathcal{E}(g)$, Id $) \leq k_{0}$.

Note that in the above statement, we can always take a smaller $\epsilon_{0}$ and a larger $k_{0}$.

Proof We set

$$
X_{n}=\left\{g \in G \mid d_{\mathcal{T}}(\mathcal{E}(g), \mathrm{Id}) \leq n\right\} .
$$

Then $G=\bigcup_{n} X_{n}=\bigcup_{n} \overline{X_{n}}$. By the Baire Category Theorem, for some $n$ the $\mathcal{T}$ interior $W$ of $\overline{X_{n}}$ in $G$ is non-empty. We choose $w \in W \cap X_{n}$ : then $d_{\mathcal{T}}(\mathcal{E}(w), \mathrm{Id}) \leq n$. Therefore Id $\in w^{-1} . W$ and $w^{-1} . W$ is the $\mathcal{T}$-interior of $w^{-1} \cdot \overline{X_{n}}$ in $G$. It follows that we can find $\epsilon_{0}>0$, such that the $\epsilon_{0}-d_{\mathcal{T}}$-neighbourhood of the identity in $G$ has a dense subset consisting of elements $g \in w^{-1}$. $\left(W \cap X_{n}\right)$. For any such $g=w^{-1} u$, we have

$$
d_{\mathcal{T}}\left(\mathcal{E}(g), \operatorname{Id}_{\mathcal{T}}\right) \leq d_{\mathcal{T}}\left(\mathcal{E}\left(w^{-1} u\right), \mathcal{E}\left(w^{-1}\right)\right)+d_{\mathcal{T}}\left(\mathcal{E}\left(w^{-1}\right), \operatorname{Id}_{\mathcal{T}}\right) \leq 2 n,
$$

since $d_{\mathcal{T}}$ is left-invariant. We set $k_{0}=2 n$.

The remainder of this section consists of more refined consequences of the Baire Category Theorem. Some readers may like to skip these until they are required in later proofs. Controlling the Teichmüller distance from the identity has no effect when dealing with a group of Möbius transformations. We need a version of Proposition 3.6 that works well when considering the group Conf of orientation preserving isometries of the Poincare disk $\mathbb{D}^{2}$. This group is conjugated to $\operatorname{PSL}(2, \mathbb{R})$ by the Cayley transform between $\mathbb{U}^{2}$ the upper halfplane and $\mathbb{D}^{2}$, the unit disk. 
3.7 Definition We use the Levi-Civita connection on the unit tangent bundle $T_{1}\left(\mathbb{D}^{2}\right)$ to the hyperbolic plane. The tangent space at a point $u \in T_{1}\left(\mathbb{D}^{2}\right)$ is the direct sum of the space of horizontal vectors at $u$ and the tangent space at $u$ to the fibres of $\pi: T_{1}\left(\mathbb{D}^{2}\right) \rightarrow \mathbb{D}^{2}$. The space of horizontal vectors at $u$ is sent by $\pi$ isomorphically to the tangent space of $\mathbb{D}^{2}$ at $\pi(u)$. Each of the direct summands has a canonical inner product, and so we obtain a canonical Riemannian metric on $T_{1}\left(\mathbb{D}^{2}\right)$.

A diffeomorphism from the unit tangent bundle to the space of triples is defined as follows. Given a unit vector $u$ at $x \in \mathbb{D}^{2}$, let $v$ be the unit orthogonal vector at $x$, such that $(u, v)$ gives the standard orientation on $\mathbb{D}^{2}$. Let $p, r \in \mathbb{S}^{1}$ be the points such that $p r$ is the directed hyperbolic geodesic through $x$ with tangent vector $v$. Let $q \in \mathbb{S}^{1}$ be the point such that $x q$ is the geodesic ray with tangent $u$. The image of $u$ is defined to be the triple $(p, q, r)$.

We have a diffeomorphism from Conf to the unit tangent bundle given by fixing some unit vector $u_{0}$, and then sending $g$ to $g u_{0}$.

3.8 Proposition The metric of Definition 3.7 is complete, and closed bounded subsets are compact. Using the diffeomorphisms of Definition 3.7, the metrics induced on Conf and on the space of triples of distinct points of $\mathbb{S}^{1}$ are complete and their closed bounded subsets are compact. The metric on Conf is left invariant, and the topology is the same as the topology of uniform convergence on maps from $\overline{\mathbb{D}^{2}}$ to itself.

Proof Since $\pi: T_{1}\left(\mathbb{D}^{2}\right) \rightarrow \mathbb{D}^{2}$ reduces Riemannian distances, it is easy to see that a Cauchy sequence in the unit tangent bundle must converge. A closed bounded subset $X$ of the unit tangent bundle projects to the closed bounded subset $\pi(X)$ of the hyperbolic plane. Now $\pi(X)$ is bounded and therefore has compact closure. So $\pi^{-1}(\pi(X))$ is contained in a compact set. It follows that $X$ is compact.

The other statements are obvious.

3.9 Proposition Let $G$ be any closed connected subgroup of Conf and let $\mathcal{E}_{G}$ : $G \rightarrow$ QC be any homomorphism such that, for each $g \in G, \mathcal{E}_{G}(g) \mid \mathbb{S}^{1}=g$. We give $G$ the metric of Definition 3.7. Then for each $\epsilon_{0}>0$, there is a $k_{0}>0$ and a dense subset $Q$ of the $\epsilon_{0}$-ball centred at the identity in $G$, such that, if $g \in Q$, then $d_{\mathcal{T}}(\mathcal{E}(g)$, Id $) \leq k_{0}$.

Proof We proceed in the same way as in the proof of Proposition 3.6, except that we use the metric of Definition 3.7 on $G$. We use the Teichmüller metric on $Q \mathcal{C}$ as before. This proves the statement for some small $\epsilon_{0}$. It can be extended to a large value of $\epsilon_{0}$ by covering the large closed ball with a finite number of small open balls. 
3.10 Corollary There is a $k_{0}>0$ with the following properties. Let $F \subset \mathbb{S}^{1}$ be a finite subset, let $C$ be the set of three possible directions of movement of a point on the circle-counter-clockwise, clockwise and fixed-and let $\alpha: F \rightarrow C$. Given $\epsilon>0$, there is a quasisymmetric $h: \mathbb{S}^{1} \rightarrow \mathbb{S}^{1}$ with the following properties:

(3.10.1) We have $d_{u}(\mathcal{E}(h)$, Id $)<\epsilon$.

(3.10.2) We have $d_{\mathcal{T}}(\mathcal{E}(h)$, Id $)<k_{0}$.

(3.10.3) For each $x \in F, h$ moves $x$ in the direction $\alpha(x)$.

Proof We may assume that the distance between any two points of $F$ is at least $3 \epsilon$. Without loss of generality we may assume that, for at least three elements $x \in F$, $\alpha(x)$ is fixed. We make a particular choice of which three fixed points to use in the discussion. We work in the groups $\mathcal{Q S}_{3}$ and $\mathcal{Q C}_{3}$ fixing the points of this particular triple. We apply Proposition 3.6, and this gives our constant $k_{0}$.

We construct $g_{i}$ inductively, obtaining a sequence $\left(g_{i}\right)_{i \in \mathbb{N}}$ in $\mathcal{L S}_{3}$, converging uniformly to the identity map of the circle, with the following properties:

(3.10.4) There is a constant $k_{0}>0$, such that, for each $i, d_{\mathcal{T}}\left(\mathcal{E}\left(g_{i}\right), \mathrm{Id}\right)<k_{0}$.

(3.10.5) For each $i$ and each $x \in F, d\left(g_{i}(x), x\right)<\epsilon$ and $d\left(g_{i}^{-1}(x), x\right)<\epsilon$.

(3.10.6) For each $i$ and each $x \in F$, the points $x, g_{i+1}^{-1}(x), g_{i}^{-1}(x)$ occur in the order $\alpha(x)$.

(3.10.7) Applying $g_{i+1}$, we see that $g_{i+1}(x), x, g_{i+1} g_{i}^{-1}(x)$ occur in order $\alpha(x)$.

(3.10.8) Going to a subsequence, we may assume, by Theorem 13.2 and Theorem 13.3 , that the sequence $\left(\mathcal{E}\left(g_{i}\right)\right)_{i \in \mathbb{N}}$ converges uniformly to a quasiconformal homeomorphism of the disk.

The sequence $\left(\mathcal{E}\left(g_{i+1}\right) \mathcal{E}\left(g_{i}\right)^{-1}\right)_{i \in \mathbb{N}}$ converges uniformly to the identity and we can choose $h=g_{i+1} g_{i}^{-1}$ for a suitably large value of $i$.

The proof of the next result is similar to that of Corollary 3.10, but is easier. Recall from Proposition 3.8 that the uniform metric on $G$ below is topologically equivalent to the metric of Definition 3.7.

3.11 Corollary There is a $k_{0}>0$ with the following property. Let $G$ be a closed connected subgroup of Conf, let $\epsilon>0$, and suppose that $x \in \mathbb{S}^{1}$ is not a fixed point for $G$. Let $\alpha(x)$ be clockwise or counter-clockwise. Then there exists $h \in G$ with the following properties:

(3.11.1) We have $d_{u}(\mathcal{E}(h)$, Id $)<\epsilon$.

(3.11.2) We have $d_{\mathcal{T}}(\mathcal{E}(h)$, Id $)<k_{0}$.

(3.11.3) $h$ moves $x$ in the direction $\alpha(x)$. 


\section{An important one-parameter group}

Let $S$ be the horizontal strip $S=(-\infty, \infty) \times(0, \pi)$ of height $\pi$. We use a strip of this height so that the euclidean and hyperbolic metrics agree on the central horizontal geodesic $y=\pi / 2$. In fact, at a point $(x, y) \in S$, infinitesimal distances satisfy $d_{\text {hyp }}=d_{\text {euc }} / \sin (y)$. It follows that, for any two points $u, v \in S$,

$$
d_{\text {hyp }}(u, v) \geq d_{\text {euc }}(u, v) .
$$

In order to define quasisymmetric homeomorphisms on the boundary of $S$ in $\mathbb{S}^{2}$, we use any conformal isomorphism $S \rightarrow \mathbb{D}^{2}$ to conjugate the situation to $\mathbb{S}^{1}$, where we apply the usual definition. Let $h$ be a quasisymmetric homeomorphism of $\partial S \cup\{+\infty,-\infty\}$, according to this definition. Suppose further that $h$ preserves each component of $\partial S$. By definition, this extends to a quasiconformal homeomorphism of $S$. By repeated reflection in the horizontal sides of the strip, we obtain a quasiconformal homeomorphism of the upper halfplane. It follows that the restriction of $h$ to either component of $\partial S$ is quasisymmetric in the usual sense of quasisymmetric homeomorphisms of $\mathbb{R}$. However, it is not true that if $f: \partial S \rightarrow \partial S$ preserves each component of $\partial S$ and is quasisymmetric in the classical sense on each component separately, then it can necessarily be extended to a quasiconformal homeomorphism of $S$.

4.1 Definition Let $r>0$. We define $v(r): \partial S \rightarrow \partial S$ by $v(r)(x, t)=(x, t)$ if $t=0$ or $t=\pi$ and $x \leq 0$, and by $v(r)(x, t)=(r x, t)$ if $t=0$ or $t=\pi$ and $x \geq 0$.

Now $v(r)$ extends by the obvious formulas to a quasiconformal homeomorphism of $S$, and so $v(r)$ is quasisymmetric. We obtain a homomorphism $v: \mathbb{R}_{+} \rightarrow Q \mathcal{S}$, from the strictly positive real numbers to the group of quasisymmetric homeomorphisms of $\partial S$, which is continuous if $Q \mathcal{S}$ is given the Teichmüller metric.

The way we are proving Theorem 1.1 is by contradiction. We make the hypothesis that we have a (possibly discontinuous) homomorphism $\mathcal{E}: \mathcal{Q S} \rightarrow Q C$ which is a one-sided inverse of $q: \mathcal{Q} \rightarrow \mathcal{Q}$, and show that this eventually leads to a contradiction.

4.2 A less restrictive hypothesis In this section we use a much less restrictive hypothesis, namely that there is an extension homomorphism $\mathcal{E}_{S}: v\left(\mathbb{R}_{+}\right) \rightarrow$ QC . The image of $v,\{v(r)\}_{r \in \mathbb{R}_{+}}$, is a continuous 1-parameter subgroup of $\mathcal{Q S}$. The image of this 1-parameter subgroup under $\mathcal{E}_{S}$ is a not necessarily continuous 1-parameter subgroup of $\mathcal{Q}$. Although the stronger hypothesis made during the overall proof is self-contradictory, the weaker hypothesis made here is easy to satisfy, and there are many ways to do so. 
4.3 Lemma Let $K$ be the maximum dilatation of $\mathcal{E}_{S}(v(2))$. Then there exist $L>0$ and $n_{0}$, depending only on $K$ and not on $\varepsilon_{S}$, with the following property. For each $n \in \mathbb{Z}$, let $R_{n} \subset S$ be the open rectangle with vertical edges of length $\pi$ and $x$-coordinates $2^{n} \pm L$. Then, for all $n \geq n_{0}$,

$$
R_{n+1} \subset \mathcal{E}_{S}(v(2))\left(R_{n}\right) .
$$

Proof By Theorem 13.6, $\mathcal{E}_{S}(v(2))$ is a $(K, \epsilon)$-quasi-isometry, for some $\epsilon>0$ depending only on $K$. It follows that there is an $L>0$, depending only on $K$, such that, for any $x_{1}>0, \mathcal{E}_{S}(v(2))\left(\left\{x_{1}\right\} \times(0, \pi)\right)$ is contained in the hyperbolic $L-$ neighbourhood (and therefore in the euclidean $L$-neighbourhood) of $\left\{2 x_{1}\right\} \times(0, \pi)$.

To understand the rest of the proof, it may be helpful to look at Figure 4.3.i. Choose $n_{0}$ so that, for $n \geq n_{0}, 2^{n}>4 L$. This will ensure that Figure 4.3.i shows the relative positions of the various components of the diagram correctly. We denote the left and right vertical boundaries of $R_{n}$ by $\lambda_{n}$ and $\rho_{n}$ respectively. Taking $x_{1}=2^{n}-L$ (so that $\left.x_{1}>0\right)$ in the last sentence of the previous paragraph, we see that every point of $\mathcal{E}_{S}(v(2))\left(\lambda_{n}\right)$ is to the left of $\lambda_{n+1}$. It also follows that every point of $\mathcal{E}_{S}(v(2))\left(\rho_{n}\right)$ is to the right of $\rho_{n+1}$. The required result follows.

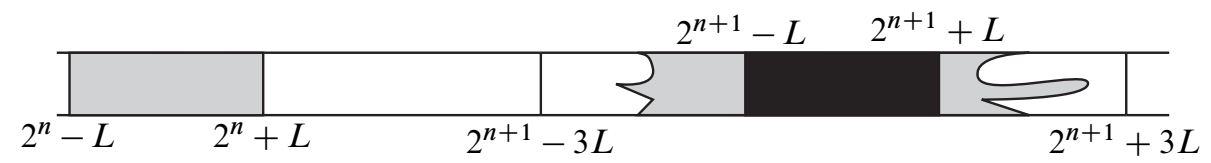

Figure 4.3.i: This picture illustrates the proof of Lemma 4.3. All the numbers shown are $x$-coordinates associated with the vertical straight lines shown. The lefthand gray region is $R_{n}$. The black region is $R_{n+1}$. The two gray regions on either side of $R_{n+1}$ show the extent of $\mathcal{E}_{S}(v(2))\left(R_{n}\right)$.

4.4 Definition We choose $L, n_{0}$ and $R_{n}$ as in the statement of Lemma 4.3. We define

$$
\ell=\bigcap_{n \geq n_{0}} \mathcal{E}_{S}\left(v(2)^{-n}\right)\left(R_{n}\right)=S \cap \bigcap_{n \geq n_{0}} \mathcal{E}_{S}\left(v(2)^{-n}\right)\left(\overline{R_{n}}\right) .
$$

4.5 Lemma The subspace $\ell$ is a closed subspace of $S . S \backslash \ell$ has exactly two components, $U_{+}$extending to $+\infty$ and $U_{-}$to $-\infty$. $U_{+}$and $U_{-}$are simply connected open subsets of $\mathbb{R}^{2}$. The closure $\bar{\ell}$ in $\mathbb{R}^{2}$ is compact and connected. It meets each component of $\partial S$ in exactly one point: $\partial S \cap \bar{\ell}=\{1,1+i \pi\}$. 
Proof We start by working with the decreasing sequence $\mathcal{E}_{S}(v(2))^{-n}\left(\overline{R_{n}}\right)$ of closed disks. The intersection of these closed disks with $\partial S$ is immediately seen to be the two-point set $\{1,1+i \pi\}$. Since the complement of each of each of these closed disks in $S$ has exactly two components, one going to $+\infty$ and the other to $-\infty$, it is easy to see that the same is true for their intersection. (Any compact curve in $S \backslash \ell$ is disjoint from all except a finite number of these disks.)

$\bar{\ell}$ is connected because the intersection of a decreasing family of compact connected subsets is connected. $U_{+}$and $U_{-}$are each components of the complement in $\mathbb{S}^{2}$ of a closed connected subset, and are therefore open and simply connected. The results stated in the lemma all follow.

4.6 Lemma Let $k>1$. Then $\mathcal{E}_{S}(v(k))(\ell)$ lies entirely to the right of $\ell$. (More rigorously, it lies entirely in the component of $S \backslash \ell$ which goes to $+\infty$.) If $r>s>0$, then $\varepsilon_{S}(v(r))(\ell)$ lies entirely to the right of $\mathcal{E}_{S}(v(s))(\ell)$. As $r$ tends to infinity, $\overline{\varepsilon_{S}(v(r))(\ell)}$ converges to $+\infty$.

Proof There is a number $L_{k}>0$, such that, for any $x>0, \mathcal{E}_{S}(v(k))$ maps the vertical geodesic joining $x$ to $x+i \pi$ into the euclidean $L_{k}$-neighbourhood of the vertical geodesic from $k x$ to $k x+i \pi$.

Let $n_{0}$ be as in Lemma 4.3. We choose $m$ large enough so that $m \geq n_{0}$, ensuring that $\mathcal{E}_{S}\left(v(2)^{m}\right)(\ell) \subset R_{m}$, and large enough so that $2^{m}+L<k\left(2^{m}-L\right)-L_{k}$. This inequality says that the righthand side $\rho_{m}$ of $R_{m}$ is to the left of $\varepsilon_{S}(v(k))\left(R_{m}\right)$. Therefore $\mathcal{E}_{S}\left(v(2)^{m}\right)(\ell)$ is to the left of $\mathcal{E}_{S}(v(k)) \mathcal{E}_{S}\left(v(2)^{m}\right)(\ell)$.

Applying $\mathcal{E}_{S}\left(v(2)^{m}\right)^{-1}$ to the terms in the final sentence of the previous paragraph, we obtain the first statement of Lemma 4.6. To obtain the second statement, we write $k=r / s$ and apply $\mathcal{E}_{S}(v(s))$ to the first statement. To prove the third statement, notice that $\varepsilon_{S}\left(v\left(2^{m}\right)\right)(\ell)$ lies to the right of $x=2^{m}-L$, which is the left edge of $R_{m}$. Using the second statement, we see that, if $r>2^{m}$, then $\mathcal{E}_{S}(v(r))(\ell)$ lies to the right of $x=2^{m}-L$.

\subsection{Corollary The interior of $\bar{\ell}$ is empty.}

Proof Lemma 4.6 shows that the various translates of $\ell$ under the group $\varepsilon_{S}\left(v\left(\mathbb{R}_{+}\right)\right)$ are disjoint closed subsets of $S$. If $\ell$ had a non-empty interior, then we would have an uncountable set of disjoint open subsets of $S$, and this is impossible.

By Lemma 4.5, $S \backslash \ell=U_{+} \cup U_{-}$, where $U_{+}$and $U_{-}$are defined in the statement of Lemma 4.5. By Corollary 4.7, $\bar{S}=\overline{U_{+}} \cup \overline{U_{-}}$. Let $W_{+}$be the interior of $S \cap \overline{U_{+}}$in $S$ and let $W_{-}$be the interior of $S \cap \overline{U_{-}}$. Then $U_{+} \subset W_{+}$and $U_{-} \subset W_{-}$. 
4.8 Definition We define $\ell_{1}=S \backslash\left(W_{+} \cup W_{-}\right)$and, for each $r>0, \ell_{r}=\mathcal{E}_{S}(v(r))\left(\ell_{1}\right)$.

\subsection{Lemma}

(4.9.1) $W_{+}$and $W_{-}$are connected.

(4.9.2) $W_{-} \cap W_{+}=\varnothing$.

(4.9.3) $\ell_{1}=S \cap \overline{W_{+}} \cap \overline{W_{-}}=S \cap \overline{U_{+}} \cap \overline{U_{-}} \subset \ell$. Later we will show that $\ell=\ell_{1}$, but this seems to be difficult to prove at the moment.

(4.9.4) $W_{+}$is equal to the interior of $S \cap \overline{W_{+}}$in $S$ and similarly for $W_{-}$.

(4.9.5) $S \cap \overline{W_{+}}=W_{+} \cup \ell_{1}$ and $S \cap \overline{W_{-}}=W_{-} \cup \ell_{1}$.

Proof To see that $W_{+}$is connected, note that any component of $W_{+}$not containing $U_{+}$must be contained in $\ell$. This would contradict Corollary 4.7.

We set $W=W_{-} \cap W_{+}$. We argue that $W=\varnothing$ by contradiction. For suppose $W \neq \varnothing$. Then $W \cap U_{+} \neq \varnothing$. Since this is a non-empty open subset of $W_{-}, W \cap U_{+} \cap U_{-} \neq \varnothing$. But this contradicts $U_{+} \cap U_{-}=\varnothing$.

We will prove (4.9.3) by showing that

$$
\ell_{1} \subset S \cap \overline{U_{+}} \cap \overline{U_{-}} \subset S \cap \overline{W_{+}} \cap \overline{W_{-}} \subset \ell_{1} .
$$

Given $p \in \ell_{1}$, we claim that $p \in \overline{U_{+}} \cap \overline{U_{-}}$. For suppose $p \notin \overline{U_{+}}$. Let $N \subset S$ be an open neighbourhood of $p$, disjoint from $\overline{U_{+}}$. Then $N \subset \overline{U_{-}}$and so $p \in N \subset W_{-}$. But this contradicts the definition of $\ell_{1}$, which proves that $\ell_{1} \subset \overline{U_{+}} \cap \overline{U_{-}}$. The inclusion $\overline{U_{+}} \cap \overline{U_{-}} \subset \overline{W_{+}} \cap \overline{W_{-}}$is obvious. Suppose now $p \in S \cap \overline{W_{+}} \cap \overline{W_{-}}$. Since $W_{+}$does not meet $W_{-}$, it does not meet $\overline{W_{-}}$. So $p \notin W_{+}$. Similarly $p \notin W_{-}$. It follows that $p \in \ell_{1}$. We also have

$$
\overline{U_{-}} \cap \overline{U_{+}} \subset \bar{S} \backslash\left(U_{+} \cup U_{-}\right)=\partial S \cup \ell .
$$

This completes the proof of (4.9.3).

To prove (4.9.4), note that, for any closed subset $Y$ in any topological space,

$$
\operatorname{int}(\overline{\operatorname{int}(Y)})=\operatorname{int}(Y) \text {. }
$$

In particular, we can apply this to $Y=\bar{U}$, where $U$ is equal to $U_{+}$or to $U_{-}$. Finally, (4.9.5) follows from (4.9.3).

4.10 Lemma $\bigcup_{r>1} \ell_{r}$ is dense in $W_{+}$. 
Proof Lemma 4.6 shows that $\bigcup_{r>1} \ell_{r} \subset W_{+}$. The last sentence of the same statement shows that, for each $x \in W_{+}$, there is some $r>0$ such that $x$ is to the left of $\ell_{r}$. $\bigcup_{r>1} \ell_{r}$ is dense in $W_{+}$, for otherwise a component of $W_{+} \backslash \overline{\bigcup_{r>1} \ell_{r}}$ would be an open connected subset with an uncountable set of disjoint translates under the homeomorphisms $\left\{\mathcal{E}_{S}(v(r))\right\}_{r>1}$.

We next prove several results, all stemming originally from the Baire category theorem, that help us to operate almost as though the homomorphism $\mathcal{E}_{S}$ were continuous.

4.11 Lemma Let $V=\mathcal{E}_{S}\left(v\left(\mathbb{R}_{+}\right)\right) \subset Q \mathcal{Q}$ and let $\bar{V}$ be its closure in the group of all homeomorphisms of $\bar{S}$ with the topology of uniform convergence on compact subsets. Then $\bar{V}$ is an abelian group. For each $u \in \bar{V}$ there is an $r>0$, such that, for each $s>0$, we have $u\left(\ell_{s}\right)=\ell_{s}$.

Proof We use the standard fact that the group of all homeomorphisms with the topology of uniform convergence on compact sets is a topological group. It is then clear that $\bar{V}$ is an abelian group.

Let $\left(r_{i}\right)_{i \in \mathbb{N}}$ be a sequence of positive real numbers, such that there is a limit $u=$ $\lim _{i \rightarrow \infty} \mathcal{E}_{S}\left(v\left(r_{i}\right)\right)$ (limits computed using uniform convergence on compact subsets of $\bar{S}$ ). By considering the effect on $\partial S$, we see that $\left(r_{i}\right)_{i \in \mathbb{N}}$ has a limit, which we denote by $r$, and that $u \mid \partial S=v(r)$. Composing with $v(1 / r)$, we may assume that $u \mid \partial S=\operatorname{Id}_{\partial S}$ and that $\left(r_{i}\right)_{i \in \mathbb{N}}$ converges to 1 .

We next show that $u\left(W_{-}\right) \subset W_{-}$. For suppose not. Then $u\left(W_{-}\right) \cap \overline{W_{+}} \neq \varnothing$ and so $u\left(W_{-}\right) \cap W_{+} \neq \varnothing$. Let $U \subset S \cap \bar{U} \subset W_{+} \cap u\left(W_{-}\right)$where $U$ is an open ball. By Lemma 4.10, there is an $s>1$ such that $\ell_{s}$ meets $U$. Now $S \cap u^{-1}(\bar{U}) \subset W_{-}$ and $\left(\mathcal{E}_{S}\left(v\left(1 / r_{i}\right)\right)\right)_{i \in \mathbb{N}}$ converges to $u^{-1}$ in the topology of uniform convergence on compact subsets. Therefore, for sufficiently large $i, \mathcal{E}_{S}\left(v\left(1 / r_{i}\right)\right)\left(\bar{U} \cap \ell_{S}\right) \subset W_{-}$. This implies that $s / r_{i}<1$. Letting $i$ tend to infinity, we find that $s \leq 1$ which is a contradiction.

Applying similar reasoning one sees that $u^{-1}\left(W_{-}\right) \subset W_{-}$, and therefore $u\left(W_{-}\right)=W_{-}$. Then $u\left(\overline{W_{-}}\right)=\overline{W_{-}}$, and so $u\left(\ell_{1}\right)=\ell_{1}$. For each $s>0, u$ commutes with $\mathcal{E}_{S}(v(s))$ and so $u\left(\ell_{s}\right)=\ell_{s}$.

4.12 Lemma For each $r>1$, there exists $k_{r} \geq 1$ and $Q_{r} \subset \mathbb{R}_{+}$, such that $Q_{r}$ is dense in and is contained in $[1 / r, r], r, 1 / r \in Q_{r}$, and, for each $s \in Q_{r}$, the maximal dilatation of $\mathcal{E}_{S}(v(s))$ is at most $k_{r}$. 
Proof The proof of Proposition 3.6 shows that the result is true when $r>1$ is near enough to 1 . We extend to all values of $r$ by taking $Q_{r}=Q_{s}^{n}$ and $k_{r}=\left(k_{s}\right)^{n}$, where $n$ is a large integer and $s=\sqrt[n]{r}$ is near to 1 .

4.13 Lemma Let $(t(i))_{i \in \mathbb{N}}$ be a sequence of real numbers converging to 1 . Then we can find a sequence $\left(E_{i}\right)_{i \in \mathbb{N}}$ with the following properties:

(4.13.1) For each $i, E_{i}$ is a quasiconformal homeomorphism of $S$. We have $d_{\mathcal{T}}\left(E_{i}, \mathrm{Id}\right) \leq 2 \log \left(k_{2}\right)$, where $k_{2} \geq 1$ is the constant of Lemma 4.12.

(4.13.2) The restriction of $E_{i}$ to $\partial S$ is equal to $v(t(i))$.

(4.13.3) Each $E_{i}$ is the uniform limit of a sequence of the form $\left(\varepsilon_{S}\left(v\left(t_{i, j}\right)\right)\right)_{j \in \mathbb{N}}$, where $\left(t_{i, j}\right)_{j \in \mathbb{N}}$ converges to $t(i)$.

(4.13.4) For each $s>0, E_{i}\left(\ell_{s}\right)=\ell_{t(i) s}$.

(4.13.5) Some subsequence of $\left(E_{i}\right)_{i \in \mathbb{N}}$ converges to the identity in the uniform metric on the space of homeomorphisms $\bar{S} \rightarrow \bar{S}$. (The uniform metric is defined using the spherical metric on $\bar{S} \subset \mathbb{S}^{2}$.)

Proof We apply Lemma 4.12 to prove (4.13.3), (4.13.1) and (4.13.2). We actually prove $d_{\mathcal{T}}\left(E_{i}\right.$, Id $) \leq \log \left(k_{2}\right)$ at this point, but we will have to replace this by $2 \log \left(k_{2}\right)$ in a moment. Then (4.13.4) follows from Lemma 4.11.

To prove (4.13.5), note that, by Theorem 13.3, we can choose a subsequence of $\left(E_{i}\right)_{i \in \mathbb{N}}$ with quasiconformal limit $u$, with $d_{\mathcal{T}}(u, \mathrm{Id}) \leq \log \left(k_{2}\right)$. By Lemma 4.11, for each $r$, $u\left(\ell_{r}\right)=\ell_{r}$. We then replace $E_{i}$ by $u^{-1} E_{i}$. It is easy to check each of the conditions in the statement for this new sequence.

4.14 Lemma $W_{+}=\bigcup_{r>1} \ell_{r}$.

Proof Let $x \in W_{+}$. From $x$ we obtain two sets of positive real numbers, namely the set of $r>0$ such that $\ell_{r}$ is strictly to the left of $x$ and the set of $r>0$ such that $\ell_{r}$ is strictly to the right of $x$. The first set is non-empty since it contains $r=1$. The second set is non-empty by the final sentence of the statement of Lemma 4.6. The two sets are disjoint intervals, and the two intervals must abut with at most one point between them. So there is a $t>0$, such that the lefthand interval has the form $(0, t)$ or $(0, t]$ and the righthand interval has the form $(t, \infty)$ or $[t, \infty)$, where $t \geq 1$.

We assume that $x$ is to the right of $\ell_{t}$ and deduce a contradiction. Let $p$ be a path from $x$ to $+\infty$ in $S \backslash \ell_{t}$ and let $\epsilon=d\left(p, \overline{\ell_{t}}\right)$. Then $\epsilon>0$. Let $(r(i))_{i \in \mathbb{N}}$ be a strictly increasing sequence in $Q_{2}$ (see Lemma 4.12), with limit 1. By Theorem 13.3, we may assume that $\left(\mathcal{E}_{S}(r(i))\right)_{i \in \mathbb{N}}$ converges uniformly on compact subsets of $\bar{S}$ to a 
$k_{2}$-quasiconformal homeomorphism $u: \bar{S} \rightarrow \bar{S}$. Then $u \mid \partial S=$ Id. For large enough $i, u \circ \mathcal{E}_{S}(v(1 / r(i)))$ moves points in $\overline{\ell_{t}}$ a distance less than $\epsilon$. In particular, the path $p$ is disjoint from

$$
u\left(\mathcal{E}_{S}(v(1 / r(i)))\left(\ell_{t}\right)\right)=u\left(\ell_{t / r(i)}\right)=\ell_{t / r(i)} .
$$

The final equality follows from Lemma 4.11. Therefore $x$ is to the right of $\ell_{t / r(i)}$. But $t / r(i)>t$, so this contradicts the definition of $t$.

Similarly if $x$ is to the left of $\ell_{t}$, we choose $(r(i))_{i \in \mathbb{N}}$ to be a strictly decreasing sequence in $Q_{2}$ with limit 1 . This time $t / r(i)<t$ and we find that $x$ is to the left of $t / r(i)$ for large enough $i$, obtaining another contradiction.

It follows that $x \in \ell_{t}$, as required.

4.15 Lemma There is only one closed subset of $\ell_{1}$ that separates $S$, namely $\ell_{1}$ itself.

Proof Let $U$ be a connected open subset of $S$ meeting $\ell_{1}$. By (4.9.3), $U$ meets $\overline{W_{-}} \cap \overline{W_{+}}$. So $W_{-} \cup U \cup W_{+}$is connected.

The next lemma is the basic a priori estimate in this paper.

\subsection{Proposition}

(4.16.1) Let $K>1$ be greater than the maximal dilatation of $\mathcal{E}_{S}(v(2))$. There is a number $W_{0}$, depending only on $K$ and not on $\mathcal{E}_{S}$, such that $\ell_{1}$ is contained in the rectangle in $S$ of height $\pi$ and width $2 W_{0}$, centred at $1+i \pi / 2$.

(4.16.2) Let $k_{2}>1$ be the constant of Lemma 4.12. Let $r_{0}>0$. Then there exists a number $W>0$, depending only on $k_{2}$ and on $r_{0}$ and not on $\mathcal{E}_{S}$, such that, for $r \geq r_{0}$, each $\ell_{r}$ is contained in the rectangle in $S$ of height $\pi$ and width $2 W$, centred at $r+i \pi / 2$.

We will apply these results in a situation where $\mathcal{E}_{S}$ changes, while $K$ and $k_{2}$ are unchanged. The statements are in their current form, in order to ensure that $W_{0}$ and $W$ will not need to be changed when $\mathcal{E}_{S}$ is changed.

Proof We will prove (4.16.2) only. The proof of (4.16.1) is the same, but simpler$k_{2}$ must be replaced by $K$ throughout. By Theorem 13.6, every $k$-quasiconformal homeomorphism is a $(k, \epsilon(k))$-quasi-isometry, where $\epsilon(k)$ is a known function of $k$. We have an $L_{2}>0$, depending only on $k_{2}$, with the following property. Let $\gamma$ be 
an arbitrary hyperbolic geodesic, with endpoints $\gamma_{+}$and $\gamma_{-}$. Then every $\left(k_{2}, \epsilon\left(k_{2}\right)\right)-$ quasi-isometry $f$ of $S$ sends $\gamma$ into the $L_{2}$-neighbourhood of the hyperbolic geodesic joining $f\left(\gamma_{+}\right)$to $f\left(\gamma_{-}\right)$. This holds in particular when $r \in Q_{2}$ (see Lemma 4.12 for the definition of $\left.Q_{2}\right)$ and $f=\mathcal{E}_{S}(v(r))$.

Let $n_{0}$ and $L$ be as in Lemma 4.3. Let $n \geq n_{0}$. By Definition 4.4, $\overline{\ell_{2^{n}}}$ is contained in the rectangle $R_{n}$ of height $\pi$ and width $2 L$, centred at $2^{n}+i \pi / 2$. We now prove Proposition 4.16 with $r \geq r_{0}=2^{n_{0}}$ and $W=L+L_{2}$. We have already dealt with the cases when $r$ is a power of 2. So we may assume that, for some $n \geq n_{0}, 2^{n}<r<2^{n+1}$. Now let $2^{n}<s<r<t<2^{n+1}$, with $s, t \in 2^{n} . Q_{2}$ and $n \geq n_{0}$. We know that $\ell_{s}$ and $\ell_{t}$ are contained in rectangles in $S$ of height $\pi$ and width $2 W$, centred at $s+i \pi / 2$ and $t+i \pi / 2$ respectively. Letting $s$ tend to $r$ from below and $t$ tend to $r$ from above, Lemma 4.6 shows that $\ell_{r}$ is contained in a rectangle of height $\pi$ and width $2 W$.

Finally, let $r_{0}>0$ satisfy $2^{n} \leq r_{0}<2^{n+1}$, where $n$ is an integer which may be negative. We may reduce to the previous case by applying an appropriate power of $\mathcal{E}_{S}(v(2))$. The maximal dilatation is therefore bounded by an appropriate power of $k_{2}$. As before, we have a quasi-isometry whose constants are bounded in terms of $n$ and $n_{0}$. The general result therefore follows.

We can now prove the following result.

4.17 Lemma We have $\ell=\ell_{1} \cdot U_{+}=W_{+}$and $U_{-}=W_{-}$are open, simply connected subspaces of $\mathbb{R}^{2}$.

Proof We show that $\ell=\ell_{1}$ by contradiction. We already know from (4.9.3) that $\ell_{1} \subset \ell$. If $\ell_{1} \neq \ell$, we have $\ell_{r} \cap \ell \neq \varnothing$ for some $r \neq 1$. Applying $\mathcal{E}(v(s))$ for a large value of $s$, we contradict (4.16.2) and Definition 4.4. The simple connectedness statement follows from Lemma 4.5.

4.18 Definition It is convenient to denote $\bigcup_{r>0} \ell_{r}$ by $\Omega_{+}$and $\bigcup_{r>0} \overline{\ell_{r}}$ by $\widehat{\Omega}_{+}$.

4.19 Lemma $\Omega_{+}$is an open simply connected subset of $S$.

Proof By Lemma 4.14,

$$
\Omega_{+}=\bigcup_{r>0} \varepsilon_{S}(v(r))\left(W_{+}\right) .
$$

As a union of open sets, $\Omega_{+}$is open. Each of the sets in the union is connected, and any two of them intersect each other non-trivially. So the union is connected. In fact $\Omega_{+}$ is simply connected, because any compact subspace is contained in $\mathcal{E}_{S}(v(r))\left(W_{+}\right)$, for some $r$, and we know from Lemma 4.17 that $W_{+}$is simply connected. 
4.20 Invariant information about $\ell_{1}$ We now reinterpret what we have found out, using the underlying hyperbolic metric. This will make it easier to transfer our results from the strip $S$ to the unit disk $\mathbb{D}^{2}$ or the upper halfplane $\mathbb{U}^{2}$. Under the conformal isomorphism exp: $S \rightarrow \mathbb{U}^{2}$ the 4 -tuple $(i \pi,-\infty, 0, \infty)$ is sent to the 4 -tuple $(-1,0,1, \infty)$ of $\mathbb{R} \cup\{\infty\}$ with cross-ratio 2 . Therefore under any conformal isomorphism of $S$ with $\mathbb{U}^{2}$, the 4-tuple $(i \pi,-\infty, 0, \infty)$ is sent to a 4tuple $\left(x_{1}, x_{2}, x_{3}, x_{4}\right)$ of cross-ratio 2 . There is a cyclic permutation $\sigma$, such that $x_{\sigma(1)}<x_{\sigma(2)}<x_{\sigma(3)}<x_{\sigma(4)} \leq \infty$. While cyclic permutations do not preserve cross-ratios in general, they do preserve cross-ratios equal to 2 .

Conversely, fixing a cyclic permutation $\sigma$ and a 4-tuple $\left(x_{1}, x_{2}, x_{3}, x_{4}\right)$ of elements of $\mathbb{R} \cup\{\infty\}$ with cross-ratio 2 and with $x_{1}<x_{2}<x_{3}<x_{4} \leq \infty$, we can define $\tau=$ $\left(x_{\sigma^{-1}(1)}, x_{\sigma^{-1}(2)}, x_{\sigma^{-1}(3)}, x_{\sigma^{-1}(4)}\right)$, thus specifying a unique conformal isomorphism $g_{\tau}: S \rightarrow \mathbb{U}^{2}$ such that $g_{\tau}(i \pi,-\infty, 0, \infty)=\tau$.

A 4-tuple of distinct points in $\mathbb{R} \cup\{\infty\}$, with cross-ratio $\times(a, b, c, d)=2$, can be characterized in several different ways. Equivalent conditions are

1) $\times(a, b, c, d)=\times(b, c, d, a)$;

2) $\times(a, b, c, d)=\times(a, d, c, b)$;

3 ) the hyperbolic geodesic $a c$ meets the hyperbolic geodesic $b d$ orthogonally.

If we map $\mathbb{U}^{2}$ conformally to $\mathbb{D}^{2}$, the cyclic permutation condition is translated to the additional condition that the four points in the 4-tuple occur in counter-clockwise order on the unit circle.

A description of the coordinates in $S$ in hyperbolic terms is obtained by taking the hyperbolic geodesic joining $\pm \infty$. Vertical geodesics are geodesics orthogonal to this main geodesic. The euclidean distance apart of vertical geodesics is equal to their hyperbolic distance from each other, so this assigns strip coordinates to all boundary points, starting from 0 and $i \pi$. We can also assign strip coordinates to non-boundary points. Such coordinates are induced on $\overline{\mathbb{U}^{2}} \cup\{\infty\}$ or on $\overline{\mathbb{D}^{2}}$ by any conformal isomorphism with $S$.

Suppose now that we have a conformal isomorphism $g_{\tau}: S \rightarrow \mathbb{D}^{2}$, corresponding to the 4-tuple $\tau=\left(a_{1}, a_{2}, a_{3}, a_{4}\right)$ of distinct counter-clockwise ordered points of $\mathbb{S}^{1}$ with cross-ratio 2 . The hyperbolic geodesics $\left(a_{3}, a_{1}\right)$ and $\left(a_{2}, a_{4}\right)$ meet orthogonally at a point we call $b_{0}$. For $t \in \mathbb{R}$, let $b_{t}$ be the point on $\left(a_{2}, a_{4}\right)$ a signed hyperbolic distance $t$ towards $a_{4}$ from $b_{0}$. For $t \in \mathbb{R}$, let $\beta_{t}$ be the geodesic through $b_{t}$ orthogonal to $\left(a_{2}, a_{4}\right)$

We have proved the following result. 
4.21 Lemma Let $2 W_{0}$ be the width of the rectangle containing $\ell_{1}$, obtained from (4.16.1). Then $W_{0}$ depends only on the maximal dilatation $K$ of $\mathcal{E}_{S}(v(2))$ and not on $\varepsilon_{S}$. The subspace $g_{\tau}\left(\ell_{1}\right)$ lies in the region between the geodesics $\beta_{1-W_{0}}$ and $\beta_{1+W_{0}}$. The geodesic $\beta_{1+W_{0}}$ divides $\mathbb{D}^{2}$ into two components, one of which contains $a_{4}$ in its boundary. This component is contained in $g_{\tau}\left(\bigcup_{r>1} \ell_{r}\right) \subset g_{\tau}\left(\Omega_{+}\right)$.

\section{Support of an extended map}

We now come to a key result (Theorem 5.1). Its proof is difficult and the route we choose for the proof is not at all obvious. We state the result and start the proof. After this start, we will, in this section, outline the way the proof proceeds, in the hope of motivating it and making it easier to follow. The proof will be completed in Section 7.

We abandon the more general hypothesis of Paragraph 4.2 and return to the standard hypothesis of Theorem 1.1, namely that there is an extension homomorphism $\mathcal{E}: \mathcal{Q S} \rightarrow$ Qe.

In Paragraph 4.20, we showed how a counter-clockwise 4-tuple $\tau$ of distinct points of $\mathbb{S}^{1}$, with cross-ratio 2 , leads to a well-defined conformal isomorphism $g_{\tau}: S \rightarrow \mathbb{D}^{2}$. Then $g_{\tau}$ can be uniquely extended to the prime ends of $S$ and $\mathbb{D}^{2}$. The space of prime ends of $S$ is equal to $\partial S \cup\{-\infty,+\infty\}$, and the space of prime ends of $\mathbb{D}^{2}$ is equal to $\mathbb{S}^{1}$.

We obtain an extension homomorphism $\mathcal{E}_{\tau}: \mathcal{Q S}(S) \rightarrow \mathcal{L} \mathcal{C}(S)$ by the formula

$$
g_{\tau} \circ \mathcal{E}_{\tau}(g) \circ g_{\tau}^{-1}=\mathcal{E}\left(g_{\tau} \circ g \circ g_{\tau}^{-1}\right) .
$$

(Note that $g_{\tau}$ on the right is really the restriction of $g_{\tau}$ to the boundary in $\mathbb{S}^{2}$ of $S$ or $\mathbb{D}^{2}$.) We can now apply the results of Section 4 , with $\varepsilon_{S}$ equal to the restriction of $\varepsilon_{\tau}$.

In Definition 4.18 we defined $\Omega_{+}$, an open connected subset of $S$, and $\widehat{\Omega}_{+}$, an open connected subset of $\bar{S}$. This construction can be carried out using $\varepsilon_{\tau}$ in place of $\varepsilon_{S}$, and the sets constructed then depend on $\tau$. We set $\Omega_{\tau}=g_{\tau}\left(\Omega_{+}\right)$and $\widehat{\Omega}_{\tau}=$ $g_{\tau}\left(\widehat{\Omega}_{+} \cup\{+\infty\}\right)$. These two sets are open and simply connected subspaces of $\mathbb{D}^{2}$ and $\overline{\mathbb{D}^{2}}$ respectively. We set $K_{\tau}=\overline{\mathbb{D}^{2}} \backslash \widehat{\Omega}_{\tau}$. This is a compact subset of the closed unit disk.

5.1 Theorem Let $C \subset \mathbb{S}^{1}$ be a closed subinterval. Then there is a closed subset $K \subset \overline{\mathbb{D}^{2}}$ such that $K \cap \mathbb{S}^{1}=C$, with the following property. Let $\psi \in Q \mathcal{S}$ be any quasisymmetric map with support in $C$. Then $\mathcal{E}(\psi)$ has support in $K$. In fact we can take $K=\bigcap_{\tau} K_{\tau}$, where $\tau$ varies over all 4-tuples $\left(c_{1}, c_{2}, c_{3}, c_{4}\right)$ arranged clockwise on $\mathbb{S}^{1}$, with cross-ratio 2 , such that $c_{1}$ and $c_{3}$ are endpoints of $C$, and $c_{2} \in C$. 
We need only show that, for each $\tau$ and each $r>0$, if $\psi \in \mathcal{L S}(S)$ is supported in $g_{\tau}^{-1}(C)=\{-\infty\} \cup(\partial S \cap\{x \leq 0\})$, then $\varepsilon_{\tau}(\psi) \mid \ell_{r}$ is the identity. From this point to the end of this section, we fix $\tau$, and this allows us to drop the subscript $\tau$ without causing confusion. In this section we will use $\mathcal{E}: \mathcal{Q S}(S) \rightarrow \mathcal{Q} \mathcal{C}(S)$ to denote the map $\mathcal{E}_{\tau}$ defined above, and $\Omega_{+}$to denote $\Omega_{\tau}$. Also in this section we reuse the symbol $C$, used in the statement of Theorem 5.1, to denote instead $\{-\infty\} \cup(\partial S \cap\{x \leq 0\})$, where it plays the same role. Let $G$ be the group of all quasisymmetric homeomorphisms of the boundary of $S$, with support in $C$.

5.2 Lemma Let $s>r>0$. Then, as $R$ tends to infinity, the euclidean distance $d_{\text {euc }}\left(\overline{\ell_{r R}}, \overline{\ell_{s R}}\right)$ tends to infinity.

Proof From Proposition 4.16, $\ell_{s R}$ is contained in a rectangle in $S$ of height $\pi$ and width $2 W$, centred at $s R+i \pi / 2$. Similarly for $\ell_{r R}$, but with the centre at $r R+i \pi / 2$. Moreover $W$ is independent of $R$. Therefore

$$
d_{\mathrm{euc}}\left(\ell_{r R}, \ell_{s R}\right) \geq(s-r) R-2 W .
$$

5.3 Lemma For each $\ell_{r}(r>0)$ and each $\psi \in G, \mathcal{E}(\psi)\left(\ell_{r}\right)=\ell_{r}$.

Proof We fix $\psi$. If we can find one value of $r>0$, such that $\mathcal{E}(\psi)\left(\ell_{r}\right)=\ell_{r}$, then the same will be true for all values of $r>0$, since $\psi$ commutes with each $v(s)$. It simplifies the argument slightly to work with a large value of $r$. We choose $r$ large enough so that any point $p \in \ell_{r}$ has two orthogonal hyperbolic geodesics through it, with all four endpoints in $\partial S \backslash C$. We fix $p$ and call the two geodesics $\gamma_{1}$ and $\gamma_{2}$. The situation is illustrated in Figure 5.3.i. Note that if $p$ is near one of the two boundary components of $S$, we may take one of the two geodesics with both endpoints in the same component of $\partial S$.

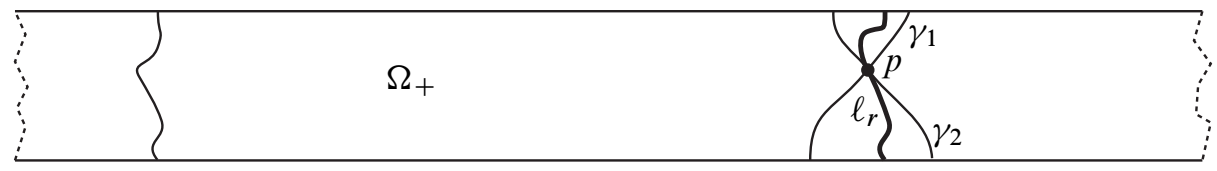

Figure 5.3.i: This diagram illustrates the proof of Lemma 5.3. Two hyperbolic geodesics $\gamma_{1}$ and $\gamma_{2}$ intersect orthogonally at $p$.

Now, for some $k>1, \mathcal{E}(\psi)$ is $k$-quasiconformal. It follows from Theorem 13.6 that $\mathcal{E}(\psi)$ is a $(k, \epsilon(k))$-quasi-isometry with respect to the hyperbolic metric on $S$, where $\epsilon(k)$ is an explicit function of $k>1$. Therefore there is a number $L>0$, depending 
only on $k$, such that, for $i=1,2, \mathcal{E}(\psi)\left(\gamma_{i}\right)$ is contained in an $L$-neighbourhood of $\gamma_{i}$. It follows that the hyperbolic distance $d(\mathcal{E}(\psi)(p), p)$ is bounded by a constant $\Delta_{1}$. This constant is independent of $p$, provided $p \in \ell_{r}$ and $r$ is large enough. By the final sentence of the statement of Lemma 4.6, we may assume that $\mathcal{E}(\psi)\left(\ell_{r}\right) \subset \Omega_{+}$.

We fix $p \in \ell_{r}$ and define $s$ by $\mathcal{E}(\psi)(p) \in \ell_{s}$. We claim that $s=r$. For suppose $s \neq r$. Using Lemma 5.2, we choose $R$ large enough so that $d_{\mathrm{euc}}\left(\ell_{R r}, \ell_{R s}\right)>\Delta_{1}$. Let $q=\mathcal{E}(v(R))(p) \in \ell_{R r}$. Then $\mathcal{E}(\psi)(q)=\mathcal{E}(v(R)) \mathcal{E}(\psi)(p) \in \ell_{s R}$. So

$$
\Delta_{1} \geq d_{\mathrm{euc}}(\mathcal{E}(\psi)(q), q) \geq d_{\mathrm{euc}}\left(\ell_{R s}, \ell_{R r}\right)>\Delta_{1},
$$

which is a contradiction.

We are now in a position to outline the proof of Theorem 5.1. The complete proof will be given later. Recall that we are denoting by $G$ the group of all quasisymmetric transformations of $\partial S \cup\{+\infty,-\infty\}$ with support in $C=(\partial S \cap\{x \leq 0\} \cup\{-\infty\}$.

We know from Lemma 5.3 that, for each $g \in G, \mathcal{E}(g)$ preserves $\ell_{r}$. A seemingly obvious approach to proving Theorem 5.1 is as follows. $\mathcal{E}$ is, in fact, equal to $\mathcal{E}_{\tau}$, depending on a 4-tuple $\tau=\left(a_{1}, a_{2}, a_{3}, a_{4}\right)$, with cross-ratio 2 , where $a_{1}$ and $a_{3}$ are the endpoints of $C$. This enables us to change $a_{2}$ to any point in the interior of $C$. Recall that the family $\left\{\ell_{r}\right\}_{r>0}$ converges to $+\infty$ as $r$ tends to $+\infty$. Changing $\tau$ enables us to change $\left\{\ell_{r}\right\}$ to another 'foliation' $\left\{\ell_{r}^{\prime}\right\}$, converging to some point in $\mathbb{R}_{+}$. Lemma 5.3 holds equally well on this new family. Therefore each $\psi \in G$ preserves each $\ell_{r} \cap \ell_{s}^{\prime}$. If this intersection is a point, it must be a fixed point for every element of $G$.

Unfortunately, we do not know that the intersection is a point, only that it is a compact subset of $\Omega_{+}$. There is no way to prove that this is a significant problem: the whole situation occurs inside an argument by contradiction, and so no counter-example is possible. However, we have worked long enough on this problem to be reasonably confident that no simple approach exists along the lines just sketched. Instead of this "obvious" but ineffective approach, we argue as follows.

Let $\operatorname{Fix}(G) \subset \bar{S}$ be the set of points fixed by all elements of $\mathcal{E}(G)$. We will prove Theorem 5.1 by contradiction. So we are assuming that $\Omega_{+}$is not a subset of Fix $(G)$. $\operatorname{Fix}(G)$ is a closed subset of $\bar{S}$ containing $\partial S \cap\{x \geq 0\}$.

The idea of the proof is to find a component of $S \backslash \operatorname{Fix}(G)$ whose closure does not meet $\partial S$. (These statements are not to be taken too literally-detailed statements in the actual proof are somewhat more convoluted.) We will show that any such component extends in $S$ to $+\infty$. Our various Baire category results enable us to find a quasisymmetric $\psi$, supported in $(\partial S \cap\{x \geq 0\}) \cup\{+\infty\}$, such that $\psi(+\infty) \in \mathbb{R}_{+}$and $\mathcal{E}(\psi)$ is near the 
identity in the compact open topology. Since $\psi$ commutes with every $g \in G$, we can show that our component of $S \backslash \operatorname{Fix}(G)$ is invariant under $\psi$. Hence our component extends to $\psi(+\infty) \in \mathbb{R}_{+}$. But we have shown that the closure of our component does not meet $\partial S$, so this is a contradiction.

Our problem then is to show that there is a component of $\Omega_{+} \backslash \operatorname{Fix}(G)$ whose closure does not meet $\partial S$. The key idea is to notice that, if each $\ell_{r}$ is a vertical interval of length $\pi$, then this is not so hard to show (though still not trivial). The basic reason that things are easier in this situation is that, by Lemma 5.3, we obtain an action of $G$ on the open vertical interval $\ell_{r}$, and we can bring the order structure of the reals into play. In particular, if we can find a compact $G$-invariant subset of the vertical open interval $\ell_{r}$, then its highest point is also $G$-invariant, and so is the lowest point. So, with this special hypothesis, it is not too hard to find our component of $\Omega_{+} \backslash \operatorname{Fix}(G)$ whose closure does not meet $\partial S$.

How do we get round the difficulty that the $\ell_{r}$ are not vertical intervals? The method is to make a model of the action in which $\ell_{r}$ is vertical. We do this by using the Riemann mapping of the simply connected open subset of $S$ to the left of $\ell_{r}$ to the subset to the left of $S \cap\{x=r\}$. There is one such Riemann mapping for each $r>0$. We have to combine this uncountable set of Riemann mappings into a coherent whole. We succeed in doing this, using the Carathéodory Kernel Theorem, and this builds a model where proofs are much easier to construct. Technical work has to be carried out, to pass back and forth between, on the one hand, the actual situation of interest, and, on the other hand, the model where the $\ell_{r}$ are vertical.

\section{The model and its properties}

We now explain how to build the model referred to at the end of Section 5.

6.1 Definition Given $r>0$, we set $U_{r}$ equal to the set of points of $S$ to the left of $\ell_{r}$. Since $\partial S \cup \overline{\ell_{r}}$ is connected, we see that $U_{r}$ is a simply connected open set. Let $S_{r}=S \cap\{x<r\}$. We set $f_{r}: U_{r} \rightarrow S_{r}$ equal to the unique conformal isomorphism fixing $-\infty, r+i \pi$ and $r$.

6.2 Definition Let $u: S \rightarrow S$ be any quasiconformal homeomorphism, preserving the family $\left\{\ell_{r}\right\}_{r>0}$. What we mean by this is that, for each $r$ there is an $s$ such that $u\left(\ell_{r}\right)=\ell_{s}$, and, furthermore, the correspondence induced on $\mathbb{R}_{+}$is a monotonic increasing homeomorphism of $\mathbb{R}_{+}$onto itself. We have come across two examples of such $u$ : by Lemma 5.3, we can take $u=\mathcal{E}(g)$ for some $g \in G$; another possibility is $u=\mathcal{E}(v(r))$ for some $r>0$. By Theorem 13.1, $u$ can be continuously extended 
to $\bar{S}$. We set $S_{+}=S \cap\{x>0\}$ and $\widehat{S_{+}}=\bar{S} \cap\{x>0\}$. We will define a map $F(u): \widehat{S_{+}} \rightarrow \widehat{S_{+}}$. Suppose $z=r+i y$ and $u\left(\ell_{r}\right)=\ell_{s}$. Let $f_{r}$ be defined as in 6.1. The map $f_{s} u f_{r}^{-1}: S_{r} \rightarrow S_{s}$ is a quasiconformal homeomorphism sending $r$ to $s, r+i \pi$ to $s+i \pi$ and $-\infty$ to $-\infty$. By Theorem 13.1, this quasiconformal homeomorphism can be continuously extended to a homeomorphism $u_{r}: \overline{S_{r}} \rightarrow \overline{S_{s}}$. We define $F(u)(z)=u_{r}(z) . F(u)$ starts life as a not necessarily continuous map of sets, but, in Proposition 6.4, we show that, for each such $u, F(u)$ is a homeomorphism.

6.3 Definition Let $H$ be the group of all $u$ as in Definition 6.2. It is easy to see that $F$ maps into the group of bijections, and that $F: H \rightarrow \operatorname{Bijections}\left(\widehat{S_{+}}\right)$is a homomorphism of groups.

6.4 Proposition For each $u$ preserving the family of sets $\left\{\ell_{r}\right\}_{r>0}$ as in Definition 6.2, $F(u): \widehat{S_{+}} \rightarrow \widehat{S_{+}}$is a homeomorphism.

Proof Since $F(u)^{-1}=F\left(u^{-1}\right)$, it is sufficient to show that, for each $u \in H, F(u)$ is continuous. Let $\left(z_{n}\right)_{n \in \mathbb{N}}$ be a sequence in $\widehat{S_{+}}$converging to a point $z_{\infty} \in \widehat{S_{+}}$. We must show that $\left(F(u)\left(z_{n}\right)\right)_{n \in \mathbb{N}}$ converges to $F(u)\left(z_{\infty}\right)$. Since $\left(z_{n}\right)_{n \in \mathbb{N}}$ is an arbitrary convergent sequence, we need only prove this convergence statement for some subsequence.

For $1 \leq n \leq \infty$, we define $r(n), y_{n}$ and $s(n)$ by the equalities $z_{n}=r(n)+i y_{n}$ and $u(r(n))=s(n)$. Then the sequence $(r(n))_{n \in \mathbb{N}}$ converges to $r(\infty)$ and $(s(n))_{n \in \mathbb{N}}$ converges to $s(\infty)$. By the Carathéodory Kernel Theorem, $\left(f_{r(n)}\right)_{n \in \mathbb{N}}$ converges to $f_{r(\infty)}$ uniformly on compact subsets of $U_{r(\infty)}$. It follows that $\left(f_{r(n)}^{-1}\right)_{n \in \mathbb{N}}$ converges to $f_{r(\infty)}^{-1}$ uniformly on compact subsets of $S_{r(\infty)}$. Similarly for $\left(f_{s(n)}\right)_{n \in \mathbb{N}}$. It follows that $\left(u_{r(n)}\right)_{n \in \mathbb{N}}$ converges to $u_{r(\infty)}$ uniformly on compact subsets of $S_{r(\infty)}$.

For $r \in \mathbb{R}$, we define $\tau_{r}: S \rightarrow S$ by $\tau_{r}(z)=z+r$. Recall the definition of $u_{r}$ from Definition 6.2. Since composition of maps in the compact-open topology is continuous, we know that

$$
\lim _{n \rightarrow \infty} \tau_{s(\infty)-s(n)} \circ u_{r(n)} \circ \tau_{r(n)-r(\infty)}=u_{r(\infty)} .
$$

The convergence is uniform on compact subsets of $S_{r(\infty)}$. But then, by Theorem 13.3, we have convergence on $\overline{S_{r(\infty)}}$, uniform with respect to the spherical metric.

Writing $z_{n}^{\prime}=z_{n}-r(n)+r(\infty)$, we have

$$
\begin{aligned}
\mid F(u)\left(z_{n}\right)- & F(u)\left(z_{\infty}\right)|=| u_{r(n)}\left(z_{n}\right)-u_{r(\infty)}\left(z_{\infty}\right) \mid \\
\leq \mid u_{r(n)}\left(z_{n}\right)- & u_{r(\infty)}\left(z_{n}^{\prime}\right)+s(\infty)-s(n)|+| s(n)-s(\infty) \mid \\
& +\left|u_{r(\infty)}\left(z_{n}^{\prime}\right)-u_{r(\infty)}\left(z_{\infty}\right)\right|
\end{aligned}
$$


The first of these three summands is equal to

$$
\tau_{s(\infty)-s(n)} u_{r(n)} \tau_{r(n)-r(\infty)}\left(z_{n}^{\prime}\right)-u_{r(\infty)}\left(z_{n}^{\prime}\right)
$$

which converges to zero. The second term clearly tends to zero. The third term tends to zero because $u_{r(\infty)}$ is continuous and $z_{n}^{\prime}$ converges to $z_{\infty}$. This proves our result.

In Proposition 6.4, we showed how to define a homomorphism of groups $F: H \rightarrow$ $\operatorname{Homeo}\left(\widehat{S_{+}}\right)$.

6.5 Lemma $F: H \rightarrow \operatorname{Homeo}\left(\widehat{S_{+}}\right)$is a continuous homomorphism, in the following sense. Let $k \geq 1$ and let $H_{k}$ be the set of elements $u \in H$ such that the maximal dilatation of $u$ is at most $k$. Then $F \mid H_{k}$ is continuous with respect to the compact-open topology.

Proof We fix $0<a<b$ and therefore the closed rectangle $R=[a, b] \times[0, \pi] \subset \widehat{S_{+}}$. Let $R^{\prime} \subset \widehat{S_{+}}$also be a closed rectangle of height $\pi$, whose interior in $\widehat{S_{+}}$contains $R$. Let $Y$ be the set of $z \in \widehat{\Omega}+$ such that $z$ lies on or between $\overline{\ell_{a}}$ and $\overline{\ell_{b}}$.

We will show that $F(v) \mid R$ depends continuously on $v$ in the compact-open topology. The proof is by contradiction. So suppose we have a $k \geq 1$, an $\epsilon>0$, a sequence $\left(z_{n}\right)_{n \in \mathbb{N}}$ in $R$ and a sequence $\left(v_{n}\right)_{n \in \mathbb{N}}$ in $H_{k}$ converging to the identity uniformly on each compact subset. We also suppose that, for each $n \geq 1, d\left(z_{n}, F\left(v_{n}\right)\left(z_{n}\right)\right) \geq \epsilon$ We may suppose that $\left(z_{n}\right)_{n \in \mathbb{N}}$ converges to some point $z_{\infty} \in R$. For each $n$ with $1 \leq n \leq \infty$, let $z_{n}=r(n)+i y_{n}$. Let $F\left(v_{n}\right)\left(z_{n}\right)=s(n)+i y_{n}^{\prime}$. Then the sequence $(r(n))_{n \in \mathbb{N}}$ converges to $r(\infty)$. Now $\left(v_{n}\right)_{n \in \mathbb{N}}$ converges to the identity, $F\left(v_{n}\right)\left|\partial S=v_{n}\right| \partial S$, and $F\left(v_{n}\right)$ sends each vertical interval to a vertical interval. Therefore $(s(n))_{n \in \mathbb{N}}$ has the same limit $r(\infty)$.

The $k$-quasiconformal homeomorphism $f_{s(n)} \circ v_{n} \circ f_{r(n)}^{-1}: S_{r(n)} \rightarrow S_{s(n)}$ extends continuously to a $k$-quasiconformal homeomorphism that we denote by $w_{n}: \overline{S_{r(n)}} \rightarrow$ $\overline{S_{S(n)}}$. Arguing as in the proof of Proposition 6.4, we find that

$$
\lim _{n \rightarrow \infty} \tau_{s(\infty)-s(n)} \circ w_{n} \circ \tau_{r(n)-r(\infty)}=\mathrm{Id} .
$$

Following (6.4.a), we see that convergence is uniform with respect to the spherical metric on $\overline{S_{r(\infty)}}$. Following (6.4.b), we see that $F\left(v_{n}\right)\left(z_{n}\right)$ converges to $z_{\infty}$. This contradiction proves our result.

6.6 Transferring prime ends between regions For each $h \in H, F(h)$ preserves the family of vertical intervals in $\widehat{S_{+}}$. Since actions on intervals are easier to understand 
than actions on open subsets of the plane, we are able to prove analogues, in the model, of results we need for the various actions on $S$ via the use of $\mathcal{E}: \mathcal{Q S}(S) \rightarrow \mathcal{Q} \mathcal{C}(S)$.

In order to use the model results in the context we desire, we need to be able to transfer readily between the situation of interest and the model. There is no conventional map of sets in either direction: the connection is via prime ends, as explained in Definition 6.2. For this reason, we give details on the transfer of prime ends from one region to another, in a general context.

Let $U$ be any simply connected proper open subset of $\mathbb{C}$ and let $f: U \rightarrow \mathbb{D}^{2}$ be a Riemann map, with inverse $g: \mathbb{D}^{2} \rightarrow U$. Given a closed interval or point $I \subset \mathbb{S}^{1}$, we define $g[I]$ to be the union of all impressions in $\mathbb{S}^{2}$ of each prime end of $U$ that corresponds under $f$ to a prime end in $I$. Square brackets are used to remind the reader that we are using notation in a specifically defined and non-standard manner.

Let $\alpha_{i}$ be an open arc in $\mathbb{D}^{2}$ and suppose $\alpha_{i}$ has two well-defined endpoints in $\mathbb{S}^{1}$, neither of them in $I$. Let $A_{i}$ be the open component of $\mathbb{D}^{2} \backslash \alpha_{i}$ whose closure contains $I$. We assume that $A_{i+1} \subset A_{i}$ and that $\bigcap_{i} \overline{A_{i}}=I$.

6.7 Lemma Let $U, I, f, g$ and $A_{i}$ be as in Paragraph 6.6. Then $g[I]=\bigcap_{i} \overline{g\left(A_{i}\right)}$ and $g[I]$ is a compact, connected subset of the frontier of $U$ in $\mathbb{S}^{2}$.

Proof When $I$ is a single point, the equality in the statement is just the definition of the impression of a prime end. In general $g[I] \subset \bigcap_{i} \overline{g\left(A_{i}\right)}$. To prove the reverse inclusion, let $z_{\infty} \in \bigcap_{i} \overline{g\left(A_{i}\right)}$. Let $\left(z_{i}\right)_{i \in \mathbb{N}}$ be a sequence in $U$ converging to $z_{\infty}$, such that, for each $i, z_{i} \in g\left(A_{i}\right)$. We must prove that $z_{\infty}$ lies in the impression of some prime end corresponding to a prime end in $I$.

Without loss of generality we may assume that $\left(f\left(z_{i}\right)\right)_{i \in \mathbb{N}}$ converges to some $p_{0} \in \overline{\mathbb{D}^{2}}$. We have $f\left(z_{i}\right) \in A_{i}$. Therefore $p_{0} \in I$. It follows that $z_{\infty}$ lies in the impression of the prime end in $U$ corresponding to $p_{0}$.

Since each $A_{i}$ is connected, so is $\overline{g\left(A_{i}\right)}$. It follows that $g[I] \subset \mathbb{S}^{2}$ is compact and connected.

The following result is easy to prove.

6.8 Lemma We continue with the notation of Paragraph 6.6. Let $\left(z_{i}\right)_{i \in \mathbb{N}}$ be a sequence of points in $\mathbb{S}^{1}$ converging to a point $z_{\infty}$. Let $V$ be any open neighbourhood of the impression $g\left[\left\{z_{\infty}\right\}\right]$ of the prime end of $U$. Then, for large enough $i, g\left[\left\{z_{i}\right\}\right] \subset$ $V$. 
6.9 Lemma Let $I \subset[r, r+i \pi]$ be a closed vertical subinterval, such that $r \notin I$. Let $f_{r}: U_{r} \rightarrow S_{r}$ be as in Definition 6.1, and let $g_{r}=f_{r}^{-1}: S_{r} \rightarrow U_{r}$. Then $g_{r}[I]$ does not meet the $x$-axis. Similarly, if $I$ does not contain $r+i \pi$, then $g_{r}[I]$ does not meet the line $y=\pi$.

Proof We construct an open rectangle $A_{i} \subset S_{r}$ in a $(1 / i)$-neighbourhood of $I$, such that $I$ is contained in the closure of the righthand vertical edge of $A_{i}$, and such that $I$ is disjoint from the closure of the lower horizontal edge of $A_{i}$. We may assume that the righthand endpoint of the lower edge of $A_{i}$ corresponds under $g_{r}$ to a singleton prime end of $U_{r}$. Then $\overline{g_{r}\left(A_{i}\right)} \cap \mathbb{R}=\varnothing$. The result follows from Lemma 6.7.

Recall that $G$ is the group of all quasisymmetric homeomorphisms of $\partial S \cup\{-\infty,+\infty\}$ supported in $(\partial S \cap\{x \leq 0\}) \cup\{-\infty\} . G$ acts on $S$ via $\mathcal{E}$. There is a second action on $S$, using $F: \mathcal{E}(G) \rightarrow \operatorname{Homeo}(S)$. We will sometimes use notation that leaves some of this structure to be understood.

\subsection{Lemma}

(6.10.1) Each component of $\Omega_{+} \backslash \operatorname{Fix}(G)$ meets each $\ell_{r}$.

(6.10.2) Each component of $S_{+} \backslash \operatorname{Fix}(F(G))$ meets each vertical interval $\{x=r\}_{r>0}$.

(6.10.3) Given $s>0$, let $U^{s}=\bigcup_{r>s} \ell_{r}$. Each component $U$ of $U^{s} \backslash \operatorname{Fix}(G)$ meets each $\ell_{r}$ with $r>s$. In particular, $+\infty$ is contained in the closure of $U$ in $\mathbb{S}^{2}$.

Proof For the first statement, let $U$ be any component. Then $U$ is open. Each element of $V=\mathcal{E}\left(v\left(\mathbb{R}_{+}\right)\right)$preserves the family $\left\{\ell_{r}\right\}_{r>0}$, as does any element of $\bar{V}-$ see Lemma 4.11. Also each element of $\bar{V}$ sends Fix $(G)$ to itself, since it commutes with $\mathcal{E}(G)$. Therefore any element of $\bar{V}$ that is sufficiently near to the identity preserves $U$. Let $J=\left\{r: \ell_{r} \cap U \neq \varnothing\right\}$. Since $U$ is connected, $J$ is a connected subset of $\mathbb{R}_{+}$, hence an interval. Using Lemma 4.13, we deduce that $J=(0, \infty)$.

By Lemma 6.5, the same proof will work for the second statement.

To prove the third statement, let $U$ be a component of $U^{s} \backslash \operatorname{Fix}(G)$. Let $J=$ $\left\{r: \ell_{r} \cap U \neq \varnothing\right\}$. We use the same argument as above, but we restrict to elements of $\bar{V}$ that move to the right on $\partial S$. If the element is sufficiently near the identity, then $U$ must be sent into itself. We deduce that $J=\left(s^{\prime}, \infty\right)$ for some $s^{\prime}$ with $s^{\prime} \geq s$. To see that $s<s^{\prime}$ leads to a contradiction, we use the same argument, but move to the left. 
We are proving Theorem 5.1 by contradiction. It follows that we can find $u \in G$, $r>0$ and $p^{\prime} \in \ell_{r}$, such that $\mathcal{E}(u)\left(p^{\prime}\right) \neq p^{\prime}$. Without loss of generality, we may assume that $p^{\prime}$ corresponds to a prime end of $U_{r}$ with a singleton impression. Let $p=f_{r}\left(p^{\prime}\right)=r+i y$. Then $F(\mathcal{E}(u))$ does not fix $p \in S \cap\{x=r\}$.

6.11 Lemma There is a minimal $F(\mathcal{E}(G))$-invariant compact subinterval of the open interval $(r, r+i \pi)$ containing $p$ in its interior. The endpoints of this interval are fixed points for the action of $F(\mathcal{E}(G))$.

Proof We set $I=[p, r+i \pi]$. By Lemma 6.9, $g[I]$ is disjoint from the $x$-axis.

To find our $F(\mathcal{E}(G))$-invariant compact interval, choose a quasisymmetric homeomorphism $\phi$ of $\partial S$ which takes $+\infty$ to $r$, such that $\phi$ is supported in $\{x>r / 2\}$. Then $\phi$ commutes with all element of $G$. If $t$ is large, $\mathcal{E}(\phi)\left(\overline{\ell_{t}}\right)$ is a compact subset $A_{p}$ separating $g_{r}[I]$ from $r$ in $\widehat{\Omega}+$. (To prove this, note that the last sentence in the statement of Lemma 4.6 shows that $\left\{\ell_{r}\right\}_{r>0}$ converges to $+\infty$ as $r$ converges to $+\infty$. Therefore $\left\{\mathcal{E}(\phi)\left(\overline{\ell_{t}}\right)\right\}_{t>0}$ converges to $\phi(+\infty)=r$ as $t$ tends to $+\infty$.)

This implies that $\ell_{r} \cap A_{p}$ separates $g[I]$ from $r$ in $\overline{\ell_{p}}$. The situation is shown in Figure 6.11.i.

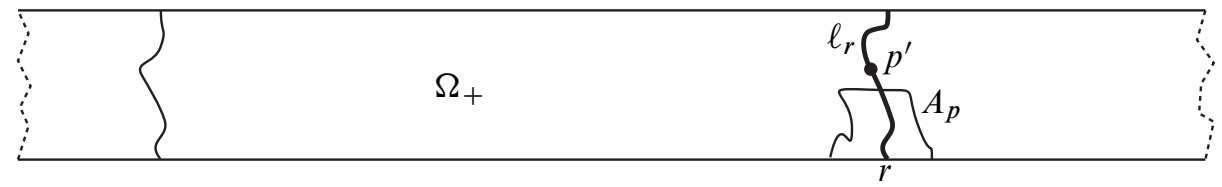

Figure 6.11.i: This diagram illustrates the proof of Lemma 6.11. We show the barrier $A_{p}$ below the point $p^{\prime}$. The situation is more complicated than it appears, because $A_{p} \cap \ell_{r}$ is not necessarily a single point.

By Lemma 5.3, $A_{p}$ is $\mathcal{E}(G)$-invariant, and so $\ell_{r} \cap A_{p}$ is $\mathcal{E}(G)$-invariant. Therefore the non-empty subset of $S \cap\{x=r\}$, corresponding to prime ends of $U_{r}$ whose impressions meet $\ell_{r} \cap A_{p}$, is $F(\mathcal{E}(G))$-invariant. By Lemma 6.8, this subset is compact. Since $F(\mathcal{E}(G))$ preserves order along $(r, r+i \pi)$, the highest point in this compact subset is fixed by $F(\mathcal{E}(G))$. We have chosen $A_{p}$ so that this highest point is below $p . A_{p}$ therefore provides a barrier around $r$ that prevents the $F(\mathcal{E}(G)$ )-orbit of $p$ reaching $r$. A similar argument applies to find a barrier $B_{p}$ around $r+i$. $\pi$. We now choose $m_{p}$ as large as possible and $M_{p}$ as small as possible so that $m_{p}<\Im(p)<M_{p}$ and such that $r+i . m_{p}$ and $r+i . M_{p}$ are fixed points for $F(\mathcal{E}(G))$. This gives our claimed minimal compact $F(\mathcal{E}(G))$-invariant interval containing $p$. 
The set of fixed points for $F(\mathcal{E}(G))$ acting on $\widehat{S_{+}}$is a closed subset containing $\partial S \cap\{x \geq 0\}$, and its complement is open in $\mathbb{C}$. Let $W$ be the component of $p$ in this complement. Then $W$ is open in $\mathbb{C}$.

6.12 Lemma There are two continuous functions $m, M: \mathbb{R}_{+} \rightarrow(0, \pi)$, such that, for each $x \in \mathbb{R}_{+}, 0<m(x)<M(x)<\pi$, and such that

$$
W=\{x+i y \in S: m(x)<y<M(x)\} .
$$

Proof Since $W$ is open, there is a $\delta>0$, such that the interval $(r-\delta, r+\delta) \times\{\mathfrak{I}(p)\} \subset$ $W$. For each $s \in(r-\delta, r+\delta)$, let $I(s)$ be the closure of the component of $s+i . \Im(p)$ in $W \cap\{x=s\}$. So $I(s)$ is a closed interval, and $I(r)=\left[r+i . m_{p}, r+i . M_{p}\right]$.

We claim that $I(s)$ depends continuously on $s$. We must show that, for an arbitrary sequence $\left(s_{i}\right)_{i \in \mathbb{N}}$ converging to $r$, there is a subsequence for which $\left(I\left(s_{i}\right)\right)_{i \in \mathbb{N}}$ converges to $I(r)$. We may suppose that the limit is some closed interval $I_{\infty}$. The openness of $W$ implies that $I(r) \subset I_{\infty}$.

We apply Lemma 4.13 with $t(i)=s_{i} / r$, to obtain a sequence $\left(E_{i}\right)_{i \in \mathbb{N}}$ of quasiconformal homeomorphisms of $S$. Taking a subsequence, we may assume that the $\left(E_{i}\right)_{i \in \mathbb{N}}$ converges uniformly to the identity on compact subsets of $\bar{S}$. By Lemma 6.5, $\left(F\left(E_{i} \mid \Omega_{+}\right)\right)_{i \in \mathbb{N}}$ also converges to the identity on compact subsets of $\bar{S} \cap\{x>0\}$. Since $E_{i}\left(\ell_{r}\right)=\ell_{s_{i}}$, it is easy to see that, for large enough $i, F\left(E_{i}\right)(I(r))=I\left(s_{i}\right)$. It follows that $I_{\infty}=I(r)$, as required.

The lower endpoint of the interval $I_{s}$, where $s \in(r-\delta, r+\delta)$, is therefore the graph of a continuous function $(r-\delta, r+\delta) \rightarrow(0, \pi)$ and the same applies to the upper endpoint of $I_{s}$. Now choose $(a, b) \subset(0, \infty)$ to be a maximal open subinterval containing $r$, for which we have continuous functions $m:(a, b) \rightarrow(0, \pi)$ and $M:(a, b) \rightarrow(0, \pi)$ that 1) extend the two functions just specified, and such that

2) each $t+i . m(t)$ and each $t+i . M(t)$ is a fixed point for all elements of $F(G)$, while

3) $t+i . y \in W$ for $m(t)<y<M(t)$.

We claim that $a=0$ and $b=\infty$. Suppose, for example, that $a>0$. Let $(a(i))_{i \in \mathbb{N}}$ decrease monotonically to $a$.

From Lemma 4.13, after replacing $(a(i))_{i \in \mathbb{N}}$ by a subsequence, we obtain a sequence $\left(E_{i}\right)_{i \in \mathbb{N}}$ of homeomorphisms such that $E_{i}\left(\ell_{a}\right)=\ell_{a(i)}$. For large $i, E_{i}$ and therefore (by Lemma 6.5) $F\left(E_{i}\right)$ are each near the identity. Since $E_{i}$ and $F\left(E_{i}\right)$ commute with $\mathcal{E}(G)$ and $F(\mathcal{E}(G))$ respectively, the graph of $m$ must be sent by $F\left(E_{i}\right)$ into the graph of $m$, except perhaps near $b$, and similarly for $M$. This is illustrated in Figure 6.12.i. Applying the inverse of $F\left(E_{i}\right)$, we see that the graphs of $m$ and $M$ can be extended 


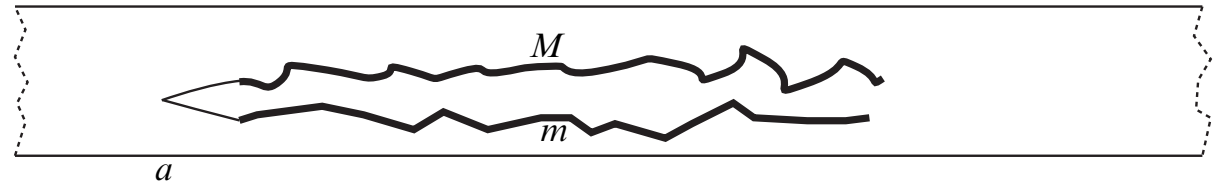

Figure 6.12.i: This diagram illustrates the middle part of the proof of Proposition 7.1. We show the graphs of the two functions $m$ and $M$. The image of each graph under $F\left(E_{i}\right)$ is shown as a thicker line.

further to the left, contradicting the maximality of $(a, b)$. A similar proof applies to $b$. This proves that $a=0$ and $b=\infty$. It follows that $W$ is the space enclosed between two graphs.

This completes the work we need to do on the model. Please refer to the end of Section 5 to get an overview of where we are in the proof of Theorem 5.1.

\section{Completion of the proof of Theorem 5.1}

Having understood the model sufficiently well, in particular, having proved Lemma 6.12, we are now in a position to complete the proof of Theorem 5.1. Recall that we are assuming that there is a point $p^{\prime} \in \Omega_{+} \backslash \operatorname{Fix}(G)$. As explained in the outline at the end of Section 5, the proof goes via the next result.

7.1 Proposition At least one of the components of $\Omega_{+} \backslash \operatorname{Fix}(G)$ has the property that its frontier in $\widehat{\Omega}_{+}$does not meet $\partial S$.

The proof of Proposition 7.1 will need a number of lemmas. The fact that a prime end may have non-trivial impression complicates the proof. For example, the points $r+i . m(r)$ and $r+i . M(r)$ in $S$ (see Lemma 6.12) are fixed under $F(G)$. However, the corresponding subsets of $\ell_{r}$ are not necessarily points, and there is no reason to believe that they are fixed under $G$, nor that they contain points of $\operatorname{Fix}(G)$.

Proof Now let $X \subset \Omega_{+}$be defined by

$$
X=\bigcup_{r>0} g_{r}[r+i . m(r), r+i . M(r)]
$$

where we are using the notation defined in Paragraph 6.6. Let $H_{1}$ be the subgroup of $\mathcal{E}\left(v\left(\mathbb{R}_{+}\right)\right)$preserving $W$. By Lemma 6.5, $H_{1}$ includes a neighbourhood in $\mathcal{E}\left(v\left(\mathbb{R}_{+}\right)\right)$ of the identity with respect to the compact-open topology on the homeomorphisms of $\bar{S}$. Let $H_{2}=\overline{H_{1}}$, where the closure is taken in the compact-open topology. Then $W$ and $\bar{W}$ are $\mathrm{H}_{2}$-invariant. 
7.2 Lemma $X$ is invariant under $\mathcal{E}(G)$ and under $H_{2} . X$ is a closed connected subset of $\widehat{\Omega}_{+}$, extending to each of the two ends of $\widehat{\Omega}_{+} . X \cap \partial S=\varnothing$. For each $r>0$, $X \cap \ell_{r} \neq \varnothing$. We have a stronger result than the connectedness of $X:$ let $J \subset \mathbb{R}_{+}$be any interval, closed, open, half-open, finite or infinite; then $X_{J}=\bigcup_{r \in J}\left\{X \cap \ell_{r}\right\}$ is connected.

Proof Given $u \in G, F(\mathcal{E}(u))$ preserves each vertical open interval $(r+i . m(r), r+$ $i . M(r)) \subset W$. Lemma 6.7 shows that $g_{r}[r+i . m(r), r+i . M(r)]$ is a compact, connected subset of $\ell_{r}$, consisting of the union of the impressions of all the prime ends of $U_{r}$, corresponding to those prime ends of $S_{r}$ given by points of the closed vertical interval $[r+i . m(r), r+i . M(r)]$. It follows that $X$ is invariant under $\mathcal{E}(u)$. The $H_{2}$-invariance of $X$ follows from that of $W$.

To see that $X$ is closed, let $\left(z_{n}\right)_{n \in \mathbb{N}}$ be a sequence in $X$ converging to a point $z_{\infty} \in \widehat{\Omega_{+}}$. We have to show that $z_{\infty} \in X$. For $1 \leq n \leq \infty$, we set $z_{n}=r(n)+i y_{n}$. There is no harm in going to a subsequence.

We set $t(n)=r(\infty) / r(n)$ and apply Lemma 4.13. We obtain a sequence $\left(E_{n}\right)_{n \in \mathbb{N}}$ of quasiconformal homeomorphisms of $\Omega_{+}$, such that $E_{n} \mid \partial S=v(t(n))$. By (4.13.5), we may assume that $\left(E_{n}\right)_{n \in \mathbb{N}}$ converges to the identity. We may therefore assume that, for each $n, E_{n} \in H_{2}$, so that $E_{n}(X)=X$. So $\left(E_{n}\left(z_{n}\right)\right)_{n \in \mathbb{N}}$ is a sequence in $X \cap \overline{\left.\ell_{r(\infty)}\right)}$, converging to $z_{\infty}$. This reduces the problem to the case where $r(n)=r(\infty)$ is independent of $n$.

By the definition of $X$, we may assume that, for $1 \leq n<\infty, z_{n}$ lies in a prime end of $U_{r(\infty)}$ corresponding to a point

$$
w_{n} \in[r(\infty)+i . m(r(\infty)), r(\infty)+i \cdot M(r(\infty))] .
$$

We may assume that $\left(w_{n}\right)_{n \in \mathbb{N}}$ converges to some point $w_{\infty}$. That $z_{\infty} \in X$ then follows from Lemma 6.8. This completes the proof that $X$ is closed.

From Lemma 6.9 and Lemma 6.12, we see that, for each $s>0, \ell_{s} \cap X$ is a compact and connected non-empty subset of $\Omega_{+}$. It follows that $X \cap \partial S=\varnothing$.

Let $J \subset \mathbb{R}_{+}$be an interval. We will show that $X_{J}$ is connected. So suppose $P \subset X_{J}$ is non-empty, and open and closed in the topology of $X_{J}$. We set $A \subset J$ equal to the set of $s$ such that $\ell_{s} \cap X=\ell_{s} \cap P$. We have just seen that, for each $s>0, \ell_{s} \cap X$ is compact, connected and non-empty. Also $\ell_{s} \cap P \subset \ell_{s} \cap X$. It follows that

$$
A=\left\{s: \ell_{s} \cap P \neq \varnothing\right\} .
$$

Let $\left(s_{i}\right)_{i \in \mathbb{N}}$ be a sequence in $A$ converging to $s \in J$. We may assume that we are working in a compact neighbourhood of $\bar{\ell}_{s}$, and therefore in a compact part of $P$. We 
deduce that $s \in A$, so that $A$ is closed. Since $P$ is open in $X_{J}$, we can apply Lemma 4.13 to show that $A$ is open in $J$. It follows that $A=J$. Therefore $P=X_{J}$. This completes the proof that $X_{J}$ is connected.

Let $U_{0}$ be the component of $\Omega_{+} \backslash X$ whose boundary contains the positive $x$-axis, and let $U_{\pi}$ be the component whose boundary contains the halfline $\{x>0, y=\pi\}$. $U_{0}$ and $U_{\pi}$ are invariant under $\mathcal{E}(G)$ and under $H_{2} . U_{0}$ and $U_{\pi}$ will be treated in the same way: we will describe the situation only for $U_{0}$.

7.3 Lemma $U_{0}$ and $U_{\pi}$ are simply connected open subspaces of $S$ that are invariant under $\mathrm{G}$ and under $\mathrm{H}_{2}$.

Proof By Lemma 4.19, $\Omega_{+}$is open and simply connected. So $\mathbb{S}^{2} \backslash \Omega_{+}$is compact and connected. Since $X$ is connected and closed in $\Omega_{+}$, and its closure in $\mathbb{S}^{2}$ contains $+\infty$, we see that $X \cup\left(\mathbb{S}^{2} \backslash \Omega_{+}\right)$is a closed and connected subspace of $\mathbb{S}^{2}$. Therefore each component of $\Omega_{+} \backslash X$ is simply connected.

In order to prove Proposition 7.1, we show that there is a component of $\Omega_{+} \backslash \operatorname{Fix}(G)$ lying between $U_{0}$ and $U_{\pi}$. This is surprisingly difficult, given that we already know the analogous result, Lemma 6.12, about the model.

What we will do is to show first that the boundary of $U_{0}$ in $\Omega_{+}$is fixed by $G$, and similarly for $U_{\pi}$, and secondly that the boundary of $U_{0}$ is disjoint from that of $U_{\pi}$. The component asked for in Proposition 7.1 is any component lying between $\overline{U_{0}}$ and $\overline{U_{\pi}}$. By Lemma 6.10, any such component will stretch from $+\infty$ to the lefthand side of $\Omega_{+}$. It can therefore be used in the manner described in the outline proof of Theorem 5.1 at the end of Section 5 .

The strategy for showing that $\partial U_{0} \subset \operatorname{Fix}(G)$ is to note that $G$ preserves $U_{0}$ and each $\ell_{r}$. $G$ fixes the lower boundary of $U_{0}$, and $\ell_{r}$ joins the lower boundary of $U_{0}$ to the upper boundary. So, naively, one might think that one can directly use this to show that the upper boundary is fixed. (It could, for example, be used correctly to show that $G$ fixes the upper boundary of $\Omega_{+}$, knowing only that the lower boundary is fixed; but we already know that the upper boundary is fixed by $G$, so that's not much help.) However, we have no control on the way that $\ell_{r}$ intersects the upper boundary of $U_{0}$, and so this outline argument is unexpectedly difficult to make rigorous and correct. Nevertheless, this is what we do. One point to watch out for is that the term prime end is relative to some definite open simply connected subset of the plane. The prime ends used in Definition 6.2 have only the most indirect relationship to the prime ends of $U_{0}$. So, in order to prove Proposition 7.1, we first prove the following the result. 
7.4 Proposition The boundary of $U_{0}$ in $\widehat{\Omega_{+}}$is contained in Fix $(G)$, and similarly for $U_{\pi}$.

Proof As usual in this paper, there is a difficulty in that prime ends may not be singletons. As usual, our reaction is to use the Riemann Mapping Theorem to transfer the data to a well-behaved situation.

Let $\Phi: U_{0} \rightarrow \mathbb{D}^{2}$ be a Riemann mapping. We normalize $\Phi$ in such a way that $\mathbb{S}^{1}$ is the disjoint union of four subsets:

1) $\{1\}$, corresponding to the end $+\infty$ of $U_{0}$;

2) $P$, a closed interval (possibly a point), arranged symmetrically about -1 , corresponding to the prime ends of $U_{0}$ at the lefthand end of $U_{0}$, arising from the end of $\widehat{\Omega}_{+}$other than $+\infty$.

3 ) an open interval $J_{0}$ containing $-i$, corresponding under $\Phi$ to the positive $x$-axis;

4) an open interval $J_{1}$ equal to the complex conjugate of $J_{0}$, corresponding to prime ends of $U_{0}$ on the upper boundary of $U_{0}$.

$H_{2}$ acts on the positive $x$-axis. By Lemma 4.13, it acts transitively on the positive $x$-axis. Conjugation by $\Phi$ does not change maximal dilatations. It follows that, if $E \in H_{2}$ is near the identity in the compact open topology on $S$, then $\Phi \circ E \circ \Phi^{-1}$ is also near the identity in the compact open topology. By Theorem 13.2, $\Phi \circ E \circ \Phi^{-1}$ extends to the closed disk. By Theorem 13.3, $\Phi \circ E \circ \Phi^{-1}$ is uniformly near the identity on $\overline{\mathbb{D}^{2}}$. We are not (yet) claiming that the action of $\Phi H_{2} \Phi^{-1}$ on $J_{1}$ is transitive.

For each $r>0$, let $C(r)$ be the component of $r$ in $\overline{\ell_{r}} \cap\left(U_{0} \cup\{y=0\}\right)$. By Lemma 13.7, $\overline{C(r)}$ meets the upper component of the boundary (in $\widehat{\Omega}_{+}$) of $U_{0}$. The following lemma is a consequence of the invariance of $X$ and the definition of $U_{0}$.

7.5 Lemma Each element of $\mathcal{E}(G)$ sends each $C(r)$ to itself. Each element of $\mathrm{H}_{2}$ preserves the family $\{C(r)\}_{r>0}$

7.6 Lemma The closure of $U_{0}$ in $\widehat{\Omega_{+}}$is equal to the disjoint union $Y=\bigcup_{r>0} \overline{C(r)}$. Also $U_{0}=\bigcup_{r>0} C(r)$.

Proof The proof needs to be carried out with care, because we do not know how $\overline{\ell_{r}}$ intersects the boundary of $U_{0}$. Since $\overline{C(r)} \subset \bar{\ell}_{r}$, the subsets are clearly disjoint.

We first show that $Y$ is closed in $\widehat{\Omega_{+}}$. Let $z_{\infty} \in \widehat{\Omega_{+}} \cap \bar{Y}$. Then there is a sequence $\left(z_{n}\right)_{n \in \mathbb{N}}$ in $U_{0}$ converging to $z_{\infty}$, such that, for each $n$ there is an $r(n)>0$ with $z_{n} \in U_{0} \cap C(r(n))$. We define $r(\infty)$ by the condition $z_{\infty} \in \ell_{r(\infty)}$. It follows that $(r(n))_{n \in \mathbb{N}}$ converges to $r(\infty)$. By Lemma 7.5 and Lemma 4.13, after going to a 
subsequence, we can find $E_{n} \in \overline{\mathcal{E}\left(v\left(\mathbb{R}_{+}\right)\right)}$such that the sequence $\left(E_{n}\right)_{n \in \mathbb{N}}$ converges uniformly to the identity in the group of homeomorphisms of $\bar{S}$ with itself, and such that $E_{n}\left(z_{n}\right) \in C_{r}(\infty)$. The sequence $\left(E_{n}\left(z_{n}\right)\right)_{n \in \mathbb{N}}$ also converges to $z_{\infty}$. It follows that $z_{\infty} \in \overline{C_{r(\infty)}}$, as required.

Next we prove that $\Phi\left(\bigcup_{r>0} C(r)\right)=\mathbb{D}^{2}$. We have just proved that $\bigcup_{r>0} C(r)$ is closed in $\mathbb{D}^{2}$.

Suppose that there is a point $p \in \mathbb{D}^{2} \backslash \Phi(Y)$. Let $r$ be the infimum of values of $s$ such that $p$ is to the left of $\overline{\Phi(C(s))}$. Since $Y$ is closed, $r$ is actually a minimum rather than an infimum. But then some element of $\Phi H_{2} \Phi^{-1}$ moves $\Phi(C(r))$ a little to the left. This contradicts the definition of $r$, and proves the final sentence of the statement.

Since $Y$ is closed and contains $U_{0}$, we see that the closure of $U_{0}$ in $\widehat{\Omega_{+}}$is contained in $Y$. The reverse inclusion is obvious, and the result follows.

$\Phi$ extends continuously to the positive $x$-axis, and we use the same name $\Phi$ for this extension. $\Phi(C(r))$ is a connected subset of $\overline{\mathbb{D}^{2}}$. We set $Y(r)=J_{1} \cap \overline{\Phi(C(r))}$. To see that $Y(r)$ is compact, we need only show that it does not extend to the ends of $J_{1}$. To see this, note that $X \cap \overline{\ell_{r}}$ is compact, and therefore can be bounded on each side by accessible prime ends in the upper boundary of $U_{0}$. It follows that $Y(r)$ lies in a compact subset of $J_{1}$ and is therefore itself compact.

We first claim that $Y(r)$ is connected. For let $\left[y^{\prime}, y^{\prime \prime}\right]$ be the smallest closed interval in $\mathbb{S}^{1}$ containing $Y(r)$, and let $z \in\left[y^{\prime}, y^{\prime \prime}\right] \backslash Y(r)$. Then $z$ cannot be connected to $J_{0}$ in $\overline{\mathbb{D}^{2}} \backslash \overline{\Phi(C(r))}$. (A similar situation is illustrated in Figure 10.8.i, where the notation is also similar to that used here, though not identical.) But we can use $\Phi H_{2} \Phi^{-1}$ to find $s>0$ such that $\overline{\Phi(C(s))}$ meets the component $Z$ of $z$ in $\left[y^{\prime}, y^{\prime \prime}\right] \backslash Y(r)$. Since $C(r) \cap C(s)=\varnothing$, we see that $\Phi(C(r)) \cap \Phi(C(s))=\varnothing$. But then $\Phi(C(s)) \cup Z$ is connected and it connects $s \in J_{0}$ to $z \in \overline{\mathbb{D}^{2}} \backslash \overline{\Phi(C(r))}$. This contradiction proves that $Y(r)$ is a point or a closed interval.

We next claim that $Y(r)$ is a singleton, proving this by contradiction. If $Y(r)$ is an interval, then, by $\mathrm{H}_{2}$-equivariance, so is $Y(s)$ for each $s>0$. Since there is an uncountable collection of such intervals, one for each $s$, it must be the case that there exist distinct $r$ and $s$, such that the corresponding open intervals intersect in an open interval $Y(r, s)$. Let $q \in Y(r, s)$ be a point corresponding to a prime end of $U_{0}$ that is accessible by a straight arc in $U_{0}$. This prime end is a singleton $\left\{q^{\prime}\right\} \subset \partial U_{0}$. So

$$
q^{\prime} \in \overline{C(r)} \cap \overline{C(s)} \subset \overline{\ell_{r}} \cap \overline{\ell_{s}}=\varnothing .
$$

This contradiction proves that $Y(r)$ is a singleton. 
Now let $z \in \partial U_{0}$ be a point accessible by a straight arc in $U_{0}$, and suppose that the corresponding prime end $z^{\prime} \in J_{1}$. By Lemma 7.6, there is an $r>0$ such that $z \in \overline{C(r)}$. We claim that $\overline{C(r)} \cap \partial U_{0}=\{r, z\}$. For suppose there is a sequence $\left(z_{n}\right)_{n \in \mathbb{N}}$ in $C(r)$ converging to some point $p \in \partial U_{0}$. where $p$ does not lie on the $x$-axis. We have shown above that $Y(r)=\left\{z^{\prime}\right\}$. Therefore $\left(\Phi\left(z_{n}\right)\right)_{n \in \mathbb{N}}$ converges to $z^{\prime}$. But then $\left(z_{n}\right)_{n \in \mathbb{N}}$ must converge to a point in the impression $\{z\}$ of the corresponding prime end in $U_{0}$. So $p=z$.

Each element $g \in \mathcal{E}(G)$ sends $\overline{C(r)}$ to itself, as we observed in Lemma 7.5. We also know that $g$ preserves $U_{0}, \partial U_{0}$ and $\partial S \cap \partial U_{0}$. Therefore $g(z)=z$. It follows that the fixed point set $\operatorname{Fix}(G)$ has a dense intersection with $\widehat{\Omega_{+}} \cap \partial U_{0}$. So $\widehat{\Omega_{+}} \cap \partial U_{0} \subset \operatorname{Fix}(G)$. Similarly, for the component $U_{\pi}$ of $\Omega_{+} \backslash X$, whose boundary contains $\{y=\pi, x>0\}$, we have $\widehat{\Omega_{+}} \cap \partial U_{\pi} \subset \operatorname{Fix}(G)$. This completes the proof of Proposition 7.4.

We continue with the proof of Proposition 7.1. We review what we know so far. $X$ is a closed connected subset of $\widehat{\Omega_{+}}$that does not meet $\partial S$, and extends from the lefthand to the righthand end of $\widehat{\Omega_{+}} . U_{0}$ and $U_{\pi}$ are components of $\Omega_{+} \backslash X$ that are non-empty, open and connected, containing respectively $\mathbb{R}_{+}$and $\mathbb{R}_{+}+i \pi$. The boundary of $U_{0}$ in $\widehat{\Omega_{+}}$is contained in $\operatorname{Fix}(G)$, and similarly for $U_{\pi}$. All the subsets mentioned in this paragraph are invariant under $G$ and $H_{2}$.

Recall from the proof of Proposition 7.1 that $X$ is defined to be the union of impressions of all prime ends corresponding to points of $\bar{W}$, where $W$ is the subset of Lemma 6.12. For each $r>0, W \cap\{x=r\}$ is a closed interval, whose interior contains points which correspond to singleton prime ends of $U_{r}$ on $\ell_{r}$. Such points of $X$ are definitely not fixed by all elements of $\mathcal{E}(G)$. It follows that $X \backslash \operatorname{Fix}(G) \neq \varnothing$.

We define $U=\Omega_{+} \backslash\left(\overline{U_{0}} \cup \overline{U_{\pi}}\right)$. We know that there is a point $x \in X$ that is not fixed by all elements of $\mathcal{E}(G)$. It is easy to see that $x \notin \overline{U_{0}} \cup \overline{U_{\pi}}$, and so $x \in U \backslash \operatorname{Fix}(G)$. The closure of any component of the non-empty open subset $U \backslash \operatorname{Fix}(G)$ is sheltered from $\partial S$ by $U_{0}$ and $U_{\pi}$. Proposition 7.1 follows.

We can now complete the proof by contradiction of Theorem 5.1, following the outline proof given at the end of Section 5. In Lemma 6.10 and Proposition 7.1 we showed the existence of a component $U^{\prime}$ of $\Omega_{+} \backslash \operatorname{Fix}(G)$, whose closure in $\widehat{\Omega}_{+}$is disjoint from $\partial S$, and which meets each $\ell_{r}$. Let $\overline{U^{\prime}}$ be the closure of $U^{\prime}$ in $\mathbb{S}^{2}$. This contains $\infty$ and is disjoint from $\partial S \cap\{x>0\}$.

Given an interval in $\mathbb{S}^{1}$, we construct a smooth one-parameter family of diffeomorphisms supported on the interval, by using a smooth vector field which is non-zero on the interior of the interval. Using a conformal isomorphism of $\mathbb{D}^{2}$ with $S$, we 
may conjugate this interval to $\partial S \cup\{+\infty\}$ so that $+\infty$ is contained in the interior of this interval. And we may assume that the interval in the boundary of $S$ lies as near as we wish to $+\infty$. By Proposition 3.6, we find a dense subset $D$ of a neighbourhood of the identity in this one-parameter subgroup, such that the quasiconformal homeomorphisms $\{\mathcal{E}(\phi): \phi \in D\}$ all have dilatation less than some constant $k$. Let $\left(\phi_{j}\right)_{j \in \mathbb{N}}$ be a sequence of distinct elements of our one-parameter group, such that $\left(\mathcal{E}\left(\phi_{j}\right)\right)_{j \in \mathbb{N}}$ converges uniformly with respect to the spherical metric on the closure of $S$ in $\mathbb{S}^{2}$. We define $\psi_{j}=\phi_{j+1} \circ \phi_{j}^{-1}$. The sequence $\left(\mathcal{E}\left(\psi_{j}\right)\right)_{j \in \mathbb{N}}$ converges uniformly to the identity with respect to the spherical metric. For each $j, \psi_{j}(+\infty) \neq+\infty$. Replacing $\psi_{j}$ by its inverse, if necessary, we may assume that $\psi_{j}(+\infty)=r_{j} \in \mathbb{R}_{+}$, where $\left(r_{j}\right)_{j \in \mathbb{N}}$ tends to $+\infty$.

Since $\psi_{j}$ commutes with $G$, we see that $\mathcal{E}\left(\psi_{j}\right)$ preserves $\operatorname{Fix}(G)$. We have to cope with the problem that $\mathcal{E}\left(\psi_{j}\right)$ does not necessarily preserve $\Omega_{+}$. Although we know that, for large enough $j, \mathcal{E}\left(\psi_{j}\right)\left(U^{\prime}\right)$ meets $U^{\prime}$, we cannot assert that these two open subsets of $S$ are equal.

For each $s>0, S \backslash \overline{U_{s}}$ is the set of points of $S$ to the right of $\ell_{s}$. Let $U^{\prime \prime}$ be a component of $U^{\prime} \cap S \backslash \overline{U_{1}}$. If $j$ is sufficiently large, $\mathcal{E}\left(\psi_{j}\right)\left(U^{\prime \prime}\right)$ is a connected subset of $\Omega_{+} \backslash \operatorname{Fix}(G)$, and so $\mathcal{E}\left(\psi_{j}\right)\left(U^{\prime \prime}\right) \subset U^{\prime}$. By Lemma 6.10, $\mathcal{E}\left(\psi_{j}\right)\left(U^{\prime \prime}\right)$ meets $\mathcal{E}\left(\psi_{j}\right)\left(\ell_{s}\right)$ for each $s>1$. Letting $s$ tend to infinity, we see that $r_{j} \in \mathbb{R}_{+}$lies in the closure of $\mathcal{E}\left(\psi_{j}\right)\left(U^{\prime \prime}\right)$, and hence in the closure of $U^{\prime}$. But this contradicts what we know about $U^{\prime}$. This completes the proof of Theorem 5.1.

\section{The dominion of an interval}

Let $(a, b) \subset \mathbb{S}^{1}$ be an open subinterval. In order for the two points $a$ and $b$ to specify an interval on the circle, we insist that the direction from $a$ to $b$ inside the interval should be counter-clockwise. The complementary interval is $[b, a]$.

Figure 8.0.i illustrates the definitions we now give. Let $G_{a, b} \subset Q \mathcal{S}$ be the group of quasisymmetric homeomorphisms supported in the closed interval $[a, b]$. We set $\operatorname{Fix}(b, a)$ equal to the set of points of the closed disk fixed under every element of $\mathcal{E}\left(G_{a, b}\right)$. $\operatorname{Fix}(b, a)$ is a closed subset of $\overline{\mathbb{D}^{2}}$ and $\operatorname{Fix}(b, a) \cap \mathbb{S}^{1}=[b, a]$. By Theorem 5.1, Fix $(b, a)$ is a closed neighbourhood of $(b, a)$ in $\overline{\mathbb{D}^{2}}$. Let $A(b, a)$ be the component of the interior (with respect to the closed disk) of Fix $(b, a)$ containing $(b, a) \subset \mathbb{S}^{1}$. We set $U(b, a)$ equal to the union of $A(b, a)$ with all bounded components of $\mathbb{C} \backslash A(b, a)$. In other words, $U(b, a)$ is obtained from $A(b, a)$ by "filling in the holes". $A(b, a)$ is a connected and $U(b, a)$ is a simply connected open subset of $\overline{\mathbb{D}^{2}}$, 

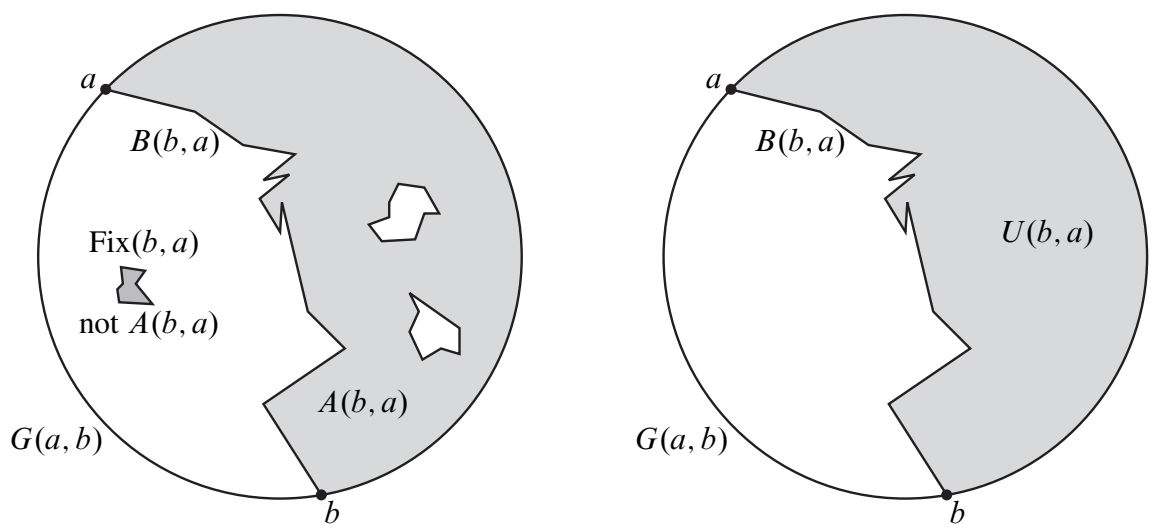

Figure 8.0.i: This diagram illustrates some of the definitions in this section. The two pictures illustrate different aspects of the same situation. The points $a$ and $b$ represent arbitrary distinct points in $\mathbb{S}^{1}$. The interval $[a, b]$ is labelled by $G(a, b)$, the group of all quasisymmetric homeomorphisms of $\mathbb{S}^{1}$ that are supported in $[a, b]$. $\operatorname{Fix}(b, a)$ consists of all fixed points for $\mathcal{E}(G(a, b))$. We have shown Fix $(a, b)$ with two components (shaded gray), but, for all we know at the moment, there could be an uncountable number of components. The open component $A(b, a)$ meets exactly one component of the fixed point set $\operatorname{Fix}(b, a)$. We obtain $U(b, a)$ by filling in all the holes in $A(b, a)$. These holes could be single points (not visible in the diagram, because they are smaller than an ink dot). $B(b, a)$ is the boundary of $U(b, a)$ in the closed disk. Note that $U(b, a)$ contains the open interval $(b, a) \subset \mathbb{S}^{1}$.

each meeting $\mathbb{S}^{1}$ in $(b, a)$. We set $B(b, a)$ equal to the frontier of $U(b, a)$ in $\overline{\mathbb{D}^{2}}$ and $D(b, a)=B(b, a) \cup U(b, a)=\overline{U(b, a)}$. We have $D(b, a) \cap \mathbb{S}^{1}=[b, a]$. The above definitions are illustrated in Figure 8.0.i.

It is conceivable that there is no inclusion relationship between $\operatorname{Fix}(b, a)$ and $D(b, a)$. It is however true that

$$
B(b, a) \subset \operatorname{Fix}(b, a) .
$$

8.1 Lemma Suppose $a, a^{\prime}, b^{\prime}, b \in \mathbb{S}^{1}$ are in counter-clockwise order. Then $D(b, a) \subset$ $D\left(b^{\prime}, a^{\prime}\right)$ and $U(b, a) \subset U\left(b^{\prime}, a^{\prime}\right)$.

Proof We have $G_{a^{\prime}, b^{\prime}} \subset G_{a, b}$. Therefore $\operatorname{Fix}(b, a) \subset \operatorname{Fix}\left(b^{\prime}, a^{\prime}\right)$ and $A(b, a) \subset$ $A\left(b^{\prime}, a^{\prime}\right)$. Hence $U(b, a) \subset U\left(b^{\prime}, a^{\prime}\right)$, and the same is true for their closures.

8.2 Definition If $x \in \mathbb{S}^{1}$, we define $F(x)=\bigcap_{x \in(b, a)} D(b, a)$, where $a$ and $b$ vary so that $x \in(b, a)$. This is the same as taking the intersection of the sets in any sequence 
$\left(D\left(b_{i}, a_{i}\right)\right)_{i \in \mathbb{N}}$, where $b_{i}$ converges to $x$ while moving in a counter-clockwise direction, and $a_{i}$ converges to $x$ while moving in a clockwise direction. We define

$$
H(x)=\bigcap_{x \in(b, a)} \overline{\mathbb{D}^{2}} \backslash U(a, b),
$$

where $a$ and $b$ vary so that $x \in(b, a)$. This is the same as taking the intersection of the sets in any sequence $\left(\overline{\mathbb{D}^{2}} \backslash U\left(a_{i}, b_{i}\right)\right)_{i \in \mathbb{N}}$, where $b_{i}$ converges to $x$ while moving in a counter-clockwise direction, and $a_{i}$ converges to $x$ while moving in a clockwise direction. We define $N(a, b)$ to be the closure in $\mathbb{C}$ of the component of $B(a, b) \backslash H(b)$ containing $a$. We illustrate $F(x)$ and $H(y)$ in Figure 8.3.i.

The following result is clear.

8.3 Lemma Let $a$ and $b$ be distinct points in $\mathbb{S}^{1} . U(a, b)$ is simply connected and open in the closed disk $\overline{\mathbb{D}^{2}}$. The subsets $D(a, b), B(a, b), F(a), H(b)$ and $N(a, b)$ are connected. $\quad F(b) \cap \mathbb{S}^{1}=H(b) \cap \mathbb{S}^{1}=\{b\}$. We have $B(b, a) \cap \mathbb{S}^{1}=\{a, b\}$, $D(b, a) \cap \mathbb{S}^{1}=[b, a]$, and $N(a, b) \cap \mathbb{S}^{1}$ is equal either to $\{a\}$ or to $\{a, b\}$.

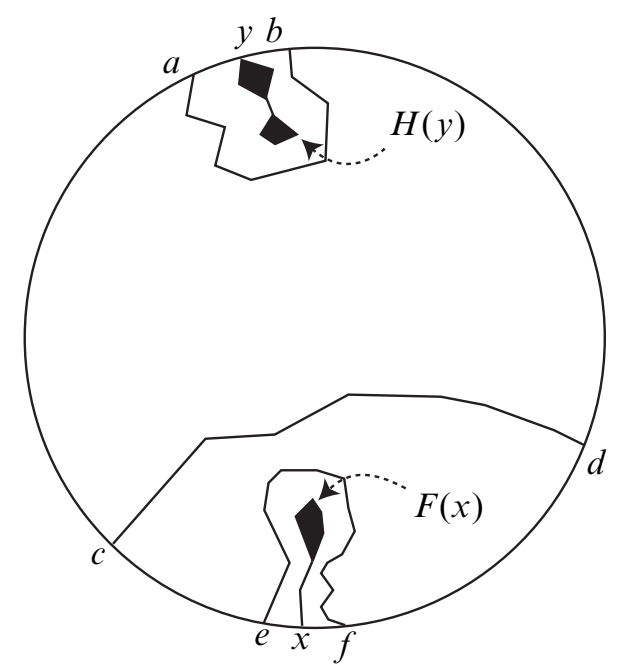

Figure 8.3.i: This picture illustrates the construction of $F(x)$ and $H(y)$. We show $B(a, b), B(c, d)$ and $B(e, f)$. The subset $D(a, b)$ lies on the same side of $B(a, b)$ as $x$. The same holds for $D(c, d)$ and $D(e, f) . F(x)$ is the intersection of subsets like $D(e, f)$, as $e$ approaches $x$ in a counterclockwise direction and $f$ approaches $x$ in a clockwise direction. $H(y)$ is the intersection of subsets like $\overline{\mathbb{D}^{2}} \backslash U(a, b)$ as $a$ approaches $y$ in a clockwise direction and $b$ approaches $y$ in a counter-clockwise direction. 
Since the sets are defined in an invariant manner, the following lemma is also immediate.

8.4 Lemma Let $\psi \in \mathcal{Q S}$. Then $\mathcal{E}(\psi) B(a, b)=B(\psi(a), \psi(b)), \mathcal{E}(\psi) D(a, b)=$ $D(\psi(a), \psi(b)), \mathcal{E}(\psi) F(x)=F(\psi(x)), \mathcal{E}(\psi) H(x)=H(\psi(x))$ and $\mathcal{E}(\psi)(N(a, b))$ $=N(\psi(a), \psi(b))$.

8.5 Lemma $D(a, b)$ and $B(a, b)$ vary continuously in the Hausdorff metric on compact subsets of $\overline{\mathbb{D}^{2}}$, as $a$ and $b$ vary in $\mathbb{S}^{1} . F(x)$ is a semicontinuous function of $x \in \mathbb{S}^{1}$ in the following sense. Let $\left(x_{i}\right)_{i \in \mathbb{N}}$ be a sequence of points in $\mathbb{S}^{1}$ converging to $x$, and let $N$ be any open neighbourhood of $F(x)$. Then, for $i$ sufficiently large, $F\left(x_{i}\right) \subset N$. The same type of semicontinuity is true for $H(x)$.

Proof We fix $a, b \in \mathbb{S}^{1}$ with $a \neq b$. Using Corollary 3.10, we construct a sequence $\left(g_{i}\right)_{i \in \mathbb{N}}$ of quasisymmetric maps, such that $\left(\mathcal{E}\left(g_{i}\right)\right)_{i \in \mathbb{N}}$ converges uniformly to the identity, and such that $\left(g_{i}(a)\right)_{i \in \mathbb{N}}$ converges to $a$ in a counter-clockwise direction and $\left(g_{i}(b)\right)_{i \in \mathbb{N}}$ converges to $b$ in a clockwise direction. It follows from Lemma 8.4 that $\left(D\left(g_{i}(a), g_{i}(b)\right)\right)_{i \in \mathbb{N}}$ converges to $D(a, b)$ and $\left(B\left(g_{i}(a), g_{i}(b)\right)\right)_{i \in \mathbb{N}}$ converges to $B(a, b)$, each in the Hausdorff sense.

We can also construct a sequence $\left(h_{i}\right)_{i \in \mathbb{N}}$ of quasisymmetric maps, such that $\left(\mathcal{E}\left(h_{i}\right)\right)_{i \in \mathbb{N}}$ converges uniformly to the identity, and such that $\left(h_{i}(a)\right)_{i \in \mathbb{N}}$ converges to $a$ in a clockwise direction and $\left(h_{i}(b)\right)_{i \in \mathbb{N}}$ converges to $b$ in a counter-clockwise direction. Then $\left(D\left(h_{i}(a), h_{i}(b)\right)\right)_{i \in \mathbb{N}}$ converges to $D(a, b)$ and $\left(B\left(h_{i}(a), h_{i}(b)\right)\right)_{i \in \mathbb{N}}$ converges to $B(a, b)$, each in the Hausdorff sense.

Given $a$ and $b$, convergence is so far proved only for very special sequences, namely those with $a_{i}=g_{i}(a), b_{i}=g_{i}(b), c_{i}=h_{i}(a)$ and $d_{i}=h_{i}(b)$. We have to do the same for general sequences. So suppose that $a^{\prime} \in\left(a_{i}, c_{i}\right)$ and $b^{\prime} \in\left(d_{i}, b_{i}\right)$ for a large value of $i$. From Lemma 8.1, we see that

$$
D\left(c_{i}, d_{i}\right) \subset D\left(a^{\prime}, b^{\prime}\right) \subset D\left(a_{i}, b_{i}\right) .
$$

Therefore $D\left(a^{\prime}, b^{\prime}\right)$ is near to $D(a, b)$. We can also see that the boundaries $B\left(c_{i}, d_{i}\right)$, $B\left(a^{\prime}, b^{\prime}\right)$ and $B\left(a_{i}, b_{i}\right)$ are near each other. This shows that $D(a, b)$ and $B(a, b)$ are continuous functions of $a$ and $b$.

To prove the semicontinuity of $F(x)$, let $N$ be an open neighbourhood of $F(x)$ in $\overline{\mathbb{D}^{2}}$ and let $\left(x_{i}\right)_{i \in \mathbb{N}}$ be a sequence of points in $\mathbb{S}^{1}$ converging to $x$. We choose $a$ and $b$ sufficiently near to $x$, with $x \in(a, b)$, so that $D(a, b) \subset N$. For $i$ sufficiently large, $x_{i} \in(a, b)$. So $F\left(x_{i}\right) \subset D(a, b) \subset N$, as required.

The semicontinuity of $H(y)$ as a function of $y$ is proved in a similar way. 
We will need the following result.

8.6 Lemma For each pair of distinct points $a$ and $b$ of $\mathbb{S}^{1}$, we have $H(b) \cap U(a, b)=$ $\varnothing$.

Proof Let $\left(b_{i}\right)_{i \in \mathbb{N}}$ be a sequence of points of $\mathbb{S}^{1}$ converging to $b$ in a clockwise direction. For each $i$, we have $H(b) \cap U\left(a, b_{i}\right)=\varnothing$. From Lemma 8.5 and Lemma 8.1 , we see that $U(a, b)=\bigcup_{i} U\left(a, b_{i}\right)$, proving our result.

\section{The case $F\left(x_{0}\right) \neq\left\{x_{0}\right\}$}

9.1 Note We fix attention on one particular $x_{0} \in \mathbb{S}^{1}$. Obviously $x_{0} \in F\left(x_{0}\right)$. There are now two possibilities: $F\left(x_{0}\right)=\left\{x_{0}\right\}$ and $F\left(x_{0}\right) \neq\left\{x_{0}\right\}$. In order to complete the proof of Theorem 1.1, we need to show that each of these two possibilities leads to a contradiction. If $F\left(x_{0}\right) \neq\left\{x_{0}\right\}$ for some $x_{0} \in \mathbb{S}^{1}$, then the same inequality is true for each $x \in \mathbb{S}^{1}$. We will assume throughout this section that, for each $x \in \mathbb{S}^{1}$, $F(x) \neq\{x\}$. The other case, when $F(x)=\{x\}$ for each $x \in \mathbb{S}^{1}$, is more difficult, and will take several more sections to deal with.

We define a function $f: \mathbb{S}^{1} \rightarrow(0,1]$ which measures how far $F(x)$ goes from $\mathbb{S}^{1}$. For each $r$ such that $0 \leq r \leq 1$, we define

$$
\operatorname{Ann}(r)=\{z \in \mathbb{C}: 1-r \leq|z| \leq 1\} .
$$

Then $\operatorname{Ann}(0)=\mathbb{S}^{1}$ and $\operatorname{Ann}(1)=\overline{\mathbb{D}^{2}}$. For $x \in \mathbb{S}^{1}$, we define

$$
f(x)=\inf \{r: F(x) \subset \operatorname{Ann}(r)\} .
$$

9.2 Lemma There is a number $\epsilon>0$, such that, for all $x \in \mathbb{S}^{1}, f(x) \geq \epsilon$.

Proof Let $X_{n}=\left\{x \in \mathbb{S}^{1}: f(x) \geq 1 / n\right\}$. By Lemma 8.5, $X_{n}$ is a compact subspace. By the Baire category theorem, there is an open interval $(a, b) \subset \mathbb{S}^{1}$ and an $n>0$, such that, for $x \in(a, b), f(x) \geq 1 / n$. It follows from Lemma 8.4 that, for each $x \in \mathbb{S}^{1}$, there is an $\epsilon_{x}>0$ and an interval $\left(a_{x}, b_{x}\right)$ containing $x$, such that, for $u \in\left(a_{x}, b_{x}\right)$, $f(u) \geq \epsilon_{x}$. Since $\mathbb{S}^{1}$ is compact, the result follows.

9.3 Lemma Let $a$ and $b$ be distinct points of $\mathbb{S}^{1}$, and let $u$ be a quasisymmetric homeomorphism, such that $u \mid[a, b]$ is the identity. Then $\mathcal{E}(u) \mid \partial F(a)$ is the identity. Suppose $v$ is a quasisymmetric homeomorphism, such that $v \|[b, a]$ is the identity. Then $\mathcal{E}(v) \mid \partial F(a)$ is the identity. 
Proof Using Corollary 3.10, we choose a sequence $\left(u_{i}\right)_{i \in \mathbb{N}}$ of quasisymmetric homeomorphisms keeping $b$ fixed, moving $a$ a small distance in the clockwise direction, such that $\left(\mathcal{E}\left(u_{i}\right)\right)_{i \in \mathbb{N}}$ converges uniformly to the identity. Then $u_{i} u u_{i}^{-1}$ is the identity on the closed interval $\left[u_{i}(a), b\right]$ and $a \in\left(u_{i}(a), b\right)$. It follows that $\mathcal{E}\left(u_{i} u u_{i}^{-1}\right)$ is the identity on $B\left(u_{i}(a), b\right)$. But $b$ can now be replaced with any point in $(a, b)$ and the hypotheses continue to hold. Therefore $\mathcal{E}\left(u_{i} u u_{i}^{-1}\right)$ is the identity on $B\left(u_{i}(a), b_{i}\right)$, where $\left(b_{i}\right)_{i \in \mathbb{N}}$ is any sequence in $(a, b)$ converging in a clockwise direction to $a$.

Letting $i$ tend to infinity, we see that $\mathcal{E}(u) \mid \partial F(a)$ is the identity. The result stated for $v$ is proved using a similar technique.

The following result is now clear.

9.4 Corollary Let $a$ and $b$ be distinct points of $\mathbb{S}^{1}$. Let $u_{1}$ be a quasisymmetric homeomorphism of $\mathbb{S}^{1}$ supported on $[a, b]$ and let $u_{2}$ be a quasisymmetric homeomorphism supported on $[b, a]$. Let $u=u_{1} \circ u_{2}$. Then $\mathcal{E}(u) \mid \partial F(a)$ is the identity.

It is convenient to work in the upper halfplane, where $a$ corresponds to 0 and $b$ to $\infty$. Let $u: \mathbb{R} \rightarrow \mathbb{R}$ be defined by $u(x)=x^{2}$ if $0 \leq x \leq 1$, by $u(x)=-x^{2}$ if $-1 \leq x \leq 0$, and by $u(x)=x$ otherwise. Using exp to move from the strip to the upper halfplane, we see that this is the same as $v(2)$ in Definition 4.1. (More precisely, the conjugating map $S \rightarrow \mathbb{U}^{2}$ is $z \mapsto-\exp (-z)$.)

Let $P_{t}$ be the parabolic transformation $x \mapsto x+t$ and suppose that $t$ is small and positive. $P_{t} \circ u$ has a fixed point $p_{t}=t+t^{2}+O\left(t^{3}\right)$, and another at $\infty$. Since the derivative of $P_{t} \circ u$ is non-zero at each of these two fixed points, we can write $P_{t} \circ u=v_{t} \circ w_{t}$, where $v_{t}$ is the identity on $\left[p_{t}, \infty\right), w_{t}$ is the identity on $\left(-\infty, p_{t}\right]$ and $v_{t}$ and $w_{t}$ are quasisymmetric. From Corollary 9.4 , it follows that $\mathcal{E}\left(P_{t}\right) \circ \mathcal{E}(u)$ is the identity on $\partial F\left(p_{t}\right)$.

Now use Corollary 3.11 to choose a sequence $(t(i))_{i \in \mathbb{N}}$, such that $\left(\varepsilon\left(P_{t(i)}\right)\right)_{i \in \mathbb{N}}$ converges uniformly to the identity on compact subsets of $\cup^{2} \cup \mathbb{R}$. We may assume that $\left(\partial F\left(p_{t(i)}\right)_{i \in \mathbb{N}}\right.$ converges in the Hausdorff metric on the space of compact subsets to a compact connected subspace $C \subset F(0)$, with $0 \in C$. By Lemma 9.2, $C \neq\{0\}$. Taking limits as $i$ tends to infinity, we see that $\mathcal{E}(u) \mid C$ is the identity.

Now $\mathcal{E}(u)$ is quasiconformal with a certain constant $K>1$. This means that, for any $x$ such that $0<x<1$, the hyperbolic geodesic from $x$ to $-x$, which is a semicircle, is mapped by $\mathcal{E}(u)$ into a quasigeodesic from $x^{2}$ to $-x^{2}$. There is an $L>0$, independent of $x$, such that the quasigeodesic is within a hyperbolic $L-$ neighbourhood of the geodesic from $x^{2}$ to $-x^{2}$. The hyperbolic distance between 
the two hyperbolic geodesics mentioned is $|\log (x)|$ which tends to infinity as $x$ tends to zero. But for $x$ small enough the two geodesics will meet $C$, and will therefore contain points that are fixed by $\mathcal{E}(u)$. So this is a contradiction. This proves that we must have $F(x)=\{x\}$ for each $x \in \mathbb{S}^{1}$.

We have reduced the proof of Theorem 1.1 to consideration of the case where, for each $x \in \mathbb{S}^{1}, F(x)=\{x\}$. This is much harder to contradict than the case $F(x) \neq\{x\}$, and the proof will occupy the next few sections.

\section{The case $F\left(x_{0}\right)=\left\{x_{0}\right\}$}

Throughout this section, we are supposing that $F\left(x_{0}\right)=\left\{x_{0}\right\}$. Then, by Lemma 8.4, $F(x)=\{x\}$ for each $x \in \mathbb{S}^{1}$.

10.1 Theorem Suppose that $F(x)=\{x\}$ for one and hence all $x \in \mathbb{S}^{1}$. Then there is an open neighbourhood $U$ of $\mathbb{S}^{1}$ in $\overline{\mathbb{D}^{2}}$ which is invariant under $\mathcal{E}(Q \mathcal{S})$. Furthermore, we can find such a $U$ with the following property: let $\left(u_{n}\right)_{n \in \mathbb{N}}$ be a sequence in $\mathcal{L}$, converging uniformly to some $u \in \mathcal{Q S}$. Suppose further that the sequence $\left(\mathcal{E}\left(u_{n}\right)\right)_{n \in \mathbb{N}}$ has uniformly bounded dilatation. Then the sequence $\left(\mathcal{E}\left(u_{n}\right)\right)_{n \in \mathbb{N}}$ converges uniformly to $\mathcal{E}(u)$ on compact subsets of $U$.

The proof of Theorem 10.1 will use a number of lemmas.

10.2 Lemma Let $a, a^{\prime}, b^{\prime}, b$ be a subset of four distinct points of $\mathbb{S}^{1}$, in counterclockwise order. Then $B(a, b)$ and $B\left(a^{\prime}, b^{\prime}\right)$ are disjoint.

Proof Let $X=B(a, b) \cap B\left(a^{\prime}, b^{\prime}\right)$. Then $X$ is a compact subset of the open disk. We will suppose that $X \neq \varnothing$ and prove a contradiction. The proof is illustrated in Figure 10.2.i.

Let $x_{1} \in\left(a^{\prime}, b^{\prime}\right)$, and let $a^{\prime}, x, x_{1}, y, b^{\prime}$ be distinct points in counter-clockwise order. Let $\psi: \mathbb{S}^{1} \rightarrow \mathbb{S}^{1}$ be a quasisymmetric homeomorphism fixing $[b, a]$ pointwise, such that $\phi\left(a^{\prime}\right)=x$ and $\phi\left(b^{\prime}\right)=y$. By Lemma 8.4, $\mathcal{E}(\phi)$ fixes $B(a, b)$ setwise, and sends $B\left(a^{\prime}, b^{\prime}\right)$ to $B(x, y)$. Recall that $D(x, y) \subset D\left(a^{\prime}, b^{\prime}\right)$ and $U\left(a^{\prime}, b^{\prime}\right) \subset U(a, b)$ by Lemma 8.1. Therefore $B(a, b) \cap U\left(a^{\prime}, b^{\prime}\right)=\varnothing$ and we have

$$
\begin{aligned}
\mathcal{E}(\phi)(X) & =B(a, b) \cap B(x, y) \subset B(a, b) \cap D(x, y) \\
& \subset B(a, b) \cap D\left(a^{\prime}, b^{\prime}\right)=B(a, b) \cap B\left(a^{\prime}, b^{\prime}\right)=X .
\end{aligned}
$$

From this we can deduce that $D(x, y) \cap X \neq \varnothing$ for all $x, y$ such that $x_{1} \in(x, y)$, and so $F\left(x_{1}\right) \cap X \neq \varnothing$. But this contradicts $F\left(x_{1}\right)=\left\{x_{1}\right\}$ and the lemma is proved. 


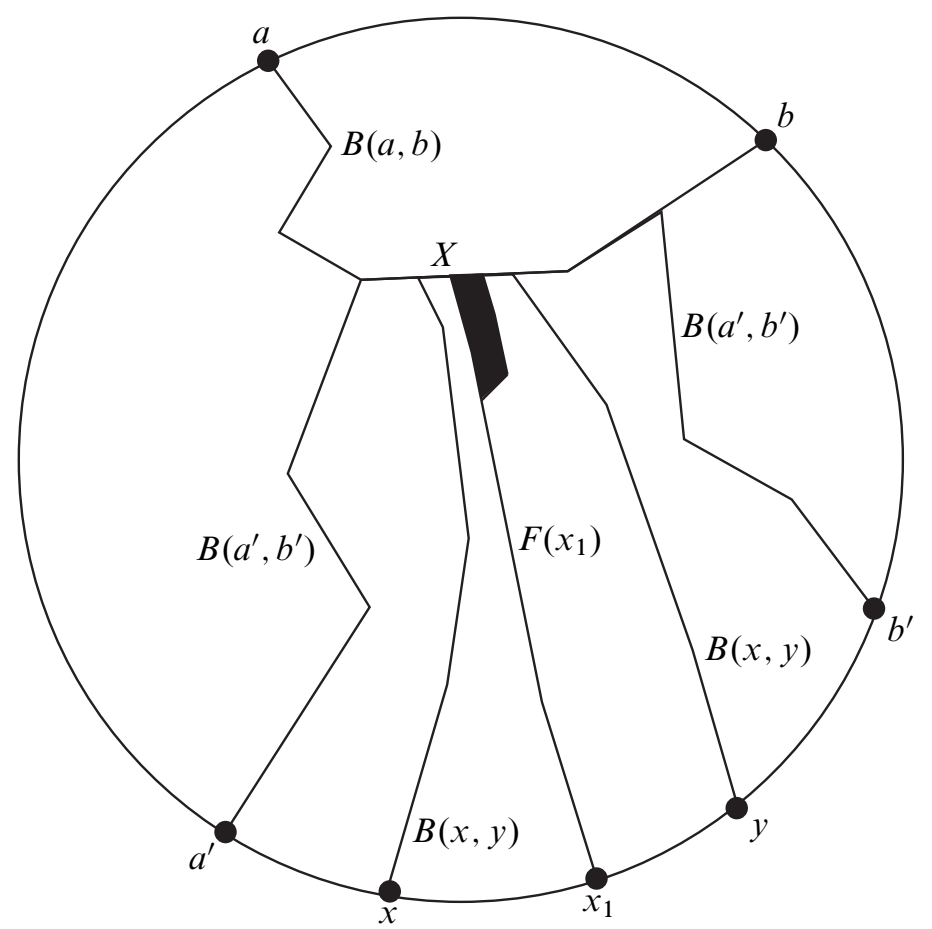

Figure 10.2.i: This picture illustrates the proof of Lemma 10.2.

10.3 Lemma Let $x_{1}$ and $x_{2}$ be distinct points in $\mathbb{S}^{1}$ and let $h$ be an orientation reversing homeomorphism of $\mathbb{S}^{1}$ fixing $x_{1}$ and $x_{2}$, and interchanging $\left(x_{1}, x_{2}\right)$ and $\left(x_{2}, x_{1}\right)$. Then $\overline{\mathbb{D}^{2}}$ is the disjoint union of the following uncountable collection of compact connected subspaces:

(10.3.1) for each $x \in\left(x_{1}, x_{2}\right)$, the set $B(x, h(x))$;

(10.3.2) $\quad H\left(x_{1}\right)$;

(10.3.3) $\left\{x_{2}\right\}$.

Proof We consider a counter-clockwise sequence of points $x_{1}, u, x, x_{2}, h(x), h(u)$, as in Figure 10.3.i. By Lemma 10.2, $D(x, h(x)) \subset U(u, h(u))$. By definition,

$$
H\left(x_{1}\right) \subset \overline{\mathbb{D}^{2}} \backslash U(u, h(u)) \subset \overline{\mathbb{D}^{2}} \backslash D(x, h(x)) .
$$

It follows that the subsets listed are indeed disjoint. The situation is illustrated in Figure 10.3.i. 


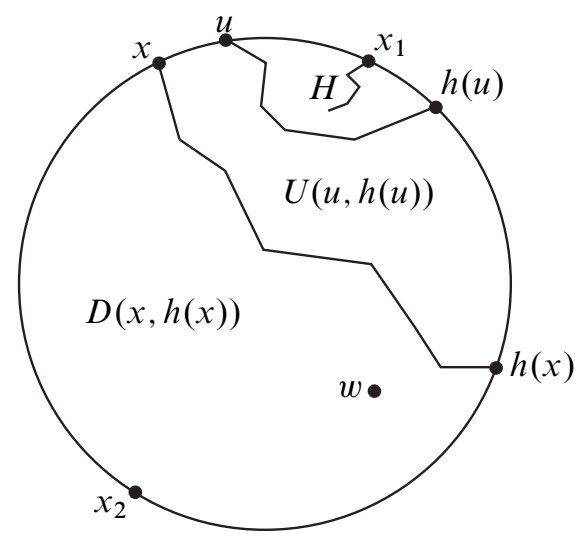

Figure 10.3.i: This picture illustrates the proof of Lemma 10.3. The set labelled $H$ is a representation of $H\left(x_{1}\right)$.

Now let $w \in \mathbb{D}^{2} \backslash H\left(x_{1}\right)$ be any point. We must show that, for some $x \in\left(x_{1}, x_{2}\right)$, we have $w \in B(x, h(x))$. Consider

$$
V=\left\{x \in\left(x_{1}, x_{2}\right): w \in U(x, h(x))\right\} .
$$

By the definition of $H\left(x_{1}\right), V$ is non-empty. By Lemma 8.5, $V$ is open. From Lemma 8.1 , we see that $V$ is an interval. We define $x_{w} \in \mathbb{S}^{1}$ by $V=\left(x_{1}, x_{w}\right) \subset\left(x_{1}, x_{2}\right)$. Since $F\left(x_{2}\right)=\left\{x_{2}\right\}$, we see that $x_{w} \neq x_{2}$. From Lemma 8.5, we see that $w \in D\left(x_{w}, h\left(x_{w}\right)\right)$. By the definition of $x_{w}, x_{w} \notin U\left(x_{w}, h\left(x_{w}\right)\right)$. We deduce that $w \in B\left(x_{w}, h\left(x_{w}\right)\right)$.

In order to prove Theorem 10.1, we need to prove that there is a $G$-invariant neighbourhood $U$ of $\mathbb{S}^{1}$ in $\overline{\mathbb{D}^{2}}$ with the following property. Let $\left(u_{n}\right)_{n \in \mathbb{N}}$ be any sequence of quasisymmetric homeomorphisms, converging uniformly to a quasisymmetric homeomorphism $u$ of $\mathbb{S}^{1}$. We assume that the dilatations of the sequence $\left(\mathcal{E}\left(u_{n}\right)\right)_{n \in \mathbb{N}}$ are uniformly bounded, as in the statement of Theorem 10.1. $U$ must have the property that the sequence converges to $\mathcal{E}(u)$, uniformly on compact subsets of $U$. Equivalently, we need only prove this result when $u=\mathrm{Id}$. From now on, we assume that $u=\operatorname{Id}$.

10.4 Definition At this point, it is convenient to fix notation for a certain quasiconformal homemorphism that we call $f: \overline{\mathbb{D}^{2}} \rightarrow \overline{\mathbb{D}^{2}}$. Using Theorem 13.3 and Theorem 13.2, we suppose that the sequence $\left(\mathcal{E}\left(u_{n}\right)\right)_{n \in \mathbb{N}}$ converges uniformly on $\overline{\mathbb{D}^{2}}$, and set $f$ equal to the limit. 
So $f$ is quasiconformal and $f \mid \mathbb{S}^{1}=\mathrm{Id}$. In order to prove Theorem 10.1, we have to define $U$, independently of $\left(u_{n}\right)_{n \in \mathbb{N}}$, and then prove that $f=\operatorname{Id}$ on $U$.

Lemma 13.7 implies that $N(a, b) \cap H(b) \neq \varnothing$. (See Definition 8.2 for $N(a, b)$.) We set

$$
A=\overline{\bigcup_{a, b} N(a, b)}
$$

and take $U$ to be the interior of $A$ in $\overline{\mathbb{D}^{2}}$.

10.5 Lemma $U$ is invariant under $\mathcal{E}(Q \mathcal{S})$ and is an open neighbourhood of $\mathbb{S}^{1}$.

Proof Since $a \in N(a, b)$, we have $\mathbb{S}^{1} \subset A$. The invariance follows from Lemma 8.4. So we need only show that $U$ is a neighbourhood of $\mathbb{S}^{1}$ - this means that, for some $\epsilon>0$, there is an annulus centred at 0 , with outer radius 1 and inner radius $1-\epsilon$, such that the open annulus is contained in $U$, or, equivalently, in $A$.

The proof is by contradiction. So suppose we have a sequence $\left(x_{i}\right)_{i \in \mathbb{N}}$ in $\mathbb{D}^{2} \backslash A$ converging to a point $x_{\infty} \in \mathbb{S}^{1}$. Now fix a small closed interval $I \subset \mathbb{S}^{1}$, just clockwise from $x_{\infty}$, and a point $a$ just counter-clockwise from $x_{\infty}$. We choose $b \in \mathbb{S}^{1}$ far from $x_{\infty}$. Let $L_{i}$ be the radial segment from $x_{i}$ to $\mathbb{S}^{1}$. By going to a subsequence, we may assume that each $L_{i}$ is disjoint from $N(a, b) \cup H(b)$.

For each $i$, we choose $\epsilon_{i}>0$ such that the euclidean distance $d\left(x_{i}, A\right)>2 \epsilon_{i} . L_{i}$ is an interval that is open at $x_{i}$ and closed at $\mathbb{S}^{1}$. The situation is illustrated in Figure 10.5.i.

We construct the universal covering $\pi_{i}: X_{i} \rightarrow \overline{\mathbb{D}^{2}} \backslash\left\{x_{i}\right\}$ by cutting along $L_{i}$, taking sheets labelled by the integers, and gluing in such a way that, when we cross $L_{i}$ in the counter-clockwise direction starting from the $n$-th sheet, we move onto the $(n+1)$-st sheet.

Now we fix $i$. Using Corollary 3.10 and a compactness argument, we construct a finite sequence of quasisymmetric maps $\left(\alpha_{k}, \alpha_{k-1}, \ldots, \alpha_{1}\right)$, such that each $\mathcal{E}\left(\alpha_{j}\right)$ moves points a euclidean distance less than $\epsilon_{i}$, and such that $\alpha_{k} \circ \ldots \circ \alpha_{1}$ fixes $b$ and sends $a$ into $I$ by a sequence of small clockwise movements. Of course, $k$ depends on $i$, and, indeed, tends to infinity as $i$ tends to infinity. Now $\mathcal{E}\left(\alpha_{k} \circ \ldots \circ \alpha_{1}\right)$ sends $N(a, b)$ to $N(x, b)$ for some $x \in I$. For each $j$ with $1 \leq j \leq k$, let $a_{j}=\alpha_{j}\left(\ldots \alpha_{1}(a)\right)$. We have a homotopy of $\mathcal{E}\left(\alpha_{j}\right)$ to the identity by moving, for each $z \in \overline{\mathbb{D}^{2}}, \mathcal{E}\left(\alpha_{j}\right)(z)$ to $z$ along a straight segment of length less than $\epsilon_{i}$. This induces a homotopy of $N\left(a_{j}, b\right)$ to $N\left(a_{j-1}, b\right)$, and the homotopy is disjoint from $x_{i}$. We compose these homotopies and lift to the covering $X_{i}$ starting from $N(a, b)$ in sheet 1 , and ending with $N\left(a_{k}, b\right)$, where $a_{k} \in I$. 


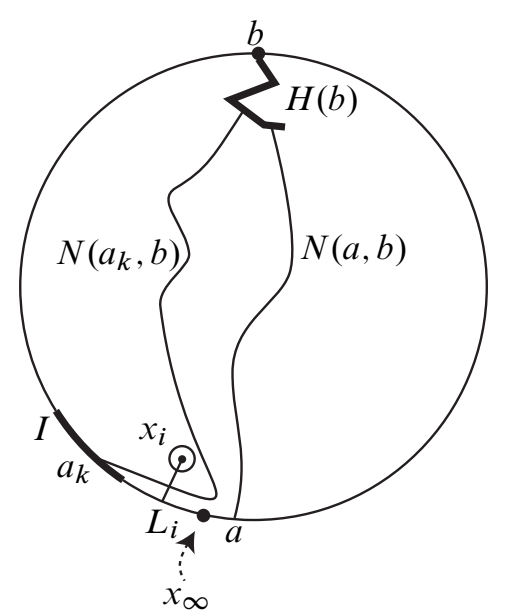

Figure 10.5.i: This illustrates the proof of Lemma 10.5. $H(b)$ and $I$ are drawn thick. The sequence $\left(x_{i}\right)_{i \in \mathbb{N}}$ converges to $x_{\infty} \in \mathbb{S}^{1}$. The disk with centre $x_{i}$ and radius $\epsilon_{i}$ is shown as a small circle. $L_{i}$ is a radial segment from $x_{i}$ to $\mathbb{S}^{1}$. The homotopy, referred to in the text, has to push $a$ clockwise to $a_{k} \in I$ "under" the sequence $\left(x_{i}\right)_{i \in \mathbb{N}}$.

To show that this leads to a contradiction, choose $p_{0} \in N(a, b) \cap H(b)$, and inductively define $p_{j}=\mathcal{E}\left(\alpha_{j}\right)\left(p_{j-1}\right)$. Then, for each $j, p_{j} \in H(b)$. Moreover, since $L_{i}$ is far from $H(b)$, after lifting to the universal cover $X_{i}$ of $\overline{\mathbb{D}^{2}} \backslash\left\{x_{i}\right\}$, we see that, for each $j$ with $1 \leq j \leq k$, part of $N\left(a_{j}, b\right)$ is in sheet 1 , namely $p_{j}$. On the other hand, having crossed $L_{i}$ once in a clockwise direction during the homotopies, $a_{k} \in N\left(a_{k}, b\right)$ is in sheet 0 . Since $N\left(a_{k}, b\right)$ is connected, it must meet $L_{i}$.

Now we let $i$ increase to infinity. We obtain a sequence $\left(y_{i}\right)_{i \in \mathbb{N}}$ in $I$, converging to a point $y_{\infty} \in I$, such that, for each $i$, there is a point $n_{i} \in N\left(y_{i}, b\right) \cap L_{i} \subset B\left(y_{i}, b\right)$. The sequence $\left(n_{i}\right)_{i \in \mathbb{N}}$ converges to $x_{\infty}$. By Lemma 8.5, $x_{\infty} \in B\left(y_{\infty}, b\right)$. But this contradicts Lemma 8.3. This completes the proof of Lemma 10.5.

10.6 Lemma Let $f: \overline{\mathbb{D}^{2}} \rightarrow \overline{\mathbb{D}^{2}}$ be the quasiconformal homeomorphism given by Definition 10.4, with $f \mid \mathbb{S}^{1}=\mathrm{Id}$, For each pair of distinct points $x, y \in \mathbb{S}^{1}$, preserves $D(x, y), B(x, y), U(x, y), F(x), H(x)$ and $N(x, y)$ setwise.

Proof Recall that $f$ is defined using a sequence $\left(u_{n}\right)_{n \in \mathbb{N}}$ of quasisymmetric homeomorphisms. From Lemma 8.5, we see that, for each $n, \mathcal{E}\left(u_{n}\right)$ sends each $D(x, y)$ to the nearby $D\left(u_{n}(x), u_{n}(y)\right)$. Taking limits, we see that $f$ preserves each $D(x, y)$. The other parts of the statement follow easily.

The following result will complete the proof of Theorem 10.1. 
10.7 Proposition Let $a$ and $b$ be distinct points of $\mathbb{S}^{1}$. Then the map $f$ is equal to the identity on the neighbourhood $N(a, b)$ of $a$ in $B(a, b)$. It follows that $f \mid A$ is the identity (where $A$ is defined just before Lemma 10.5).

Proof We have already proved a result (Proposition 7.4) that is very similar to this. Some discussion is needed to set up the parallel and provide a convincing proof in the current situation.

By Lemma 8.6, we know that $H(b) \cap U(a, b)=\varnothing$. Let $h: \mathbb{S}^{1} \rightarrow \mathbb{S}^{1}$ be induced by the hyperbolic isometry of $\mathbb{D}^{2}$ given by hyperbolic reflection in the hyperbolic geodesic $(a, b)$.

By Lemma 10.3 (with $h^{-1}$ replacing $h$ ), the disjoint subsets $\{B(h(x), x)\}_{x \in(a, b)}$ fill up $\overline{\mathbb{D}^{2}} \backslash H(b)$. See Figure 10.7.i for a picture. In particular $U(a, b) \subset \bigcup_{x \in(a, b)} B(h(x), x)$. For each $x \in(a, b)$, let $C(x)$ be the component of $U(a, b)) \cap B(h(x), x)$ containing $x$. It follows from Lemma 13.7 that $\overline{C(x)} \cap B(a, b) \neq \varnothing$.

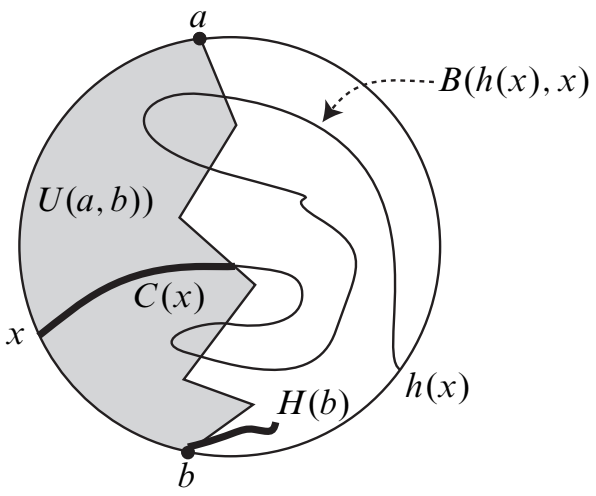

Figure 10.7.i: This picture illustrates the beginning of the proof of Proposition 10.7. $U(a, b)$ is shaded grey. $B(h(x), x)$ is pointed to by a dotted arrow. $C(x) \subset B(h(x), x) \cap U(a, b)$ is drawn as a thicker line, as is $H(b)$.

For $t \in \mathbb{R}$, let $\Phi_{t}: \mathbb{D}^{2} \rightarrow \mathbb{D}^{2}$ be the hyperbolic transformation with fixed points $a$ and $b$, moving points of the geodesic from $a$ to $b$ a signed hyperbolic distance $t$ towards $b$ and away from $a$. Let $\phi_{t}: \mathbb{S}^{1} \rightarrow \mathbb{S}^{1}$ be induced by $\Phi_{t}$. The subgroup $\left\{\mathcal{E}\left(\phi_{t}\right)\right\}_{t \in \mathbb{R}}$ preserves $B(a, b)$, but the dependence on $t$ is not necessarily continuous. For $x \in(a, b)$, we have $\mathcal{E}\left(\phi_{t}\right)(C(x))=C\left(\phi_{t}(x)\right)$.

Here $a$ plays the role of $+\infty$ in Lemma 7.6, and $(a, b)$ plays the role of $\mathbb{R}_{+}$. The group $\left\{\mathcal{E}\left(\phi_{t}\right)\right\}_{t \in \mathbb{R}}$ here plays the role of $\{\mathcal{E}(v(r))\}_{r>0}$ there. $B(h(x), x)$ here plays 
the role of $\ell_{r}$ there. $C(x)$ here plays the role of $C(r)$ there. The use of Lemma 4.13 used in the proof of Lemma 7.6 is replaced by the use here of Corollary 3.11.

Let $C$ be the component of $a$ in $B(a, b) \backslash H(b)$. We define $\widehat{U}=U(a, b) \cup C$, in analogy to $\widehat{\Omega_{+}}$.

10.8 Lemma The closure of $U(b, a)$ in $\widehat{U}$ is equal to the disjoint union $Y=$ $\bigcup_{x \in(a, b)} \overline{C(x)}$. Also $U(a, b)=\bigcup_{x \in(a, b)} C(x)$.

Proof The proof is just a repeat of the proof of Lemma 7.6, using the dictionary above to translate to this situation..

We can now complete the proof of Proposition 10.7. The proof follows exactly the lines of the proof of Proposition 7.4. Let $V$ be the interior of $D(a, b)$ as a subset of $\mathbb{C}$. So $U(a, b)=(a, b) \cup V$. Let $L$ be an open halfdisk. Let $R: V \rightarrow L$ be a Riemann mapping. We choose $R$ so that the circular part $L_{c} \subset \partial L$ corresponds to $[a, b]=\mathbb{S}^{1} \cap D(a, b)$ and the straight part $L_{s} \subset \partial L$ corresponds to $B(a, b) . R$ extends continuously to $[a, b] \subset \mathbb{S}^{1}$, and we use the same notation $R$ for the extension.

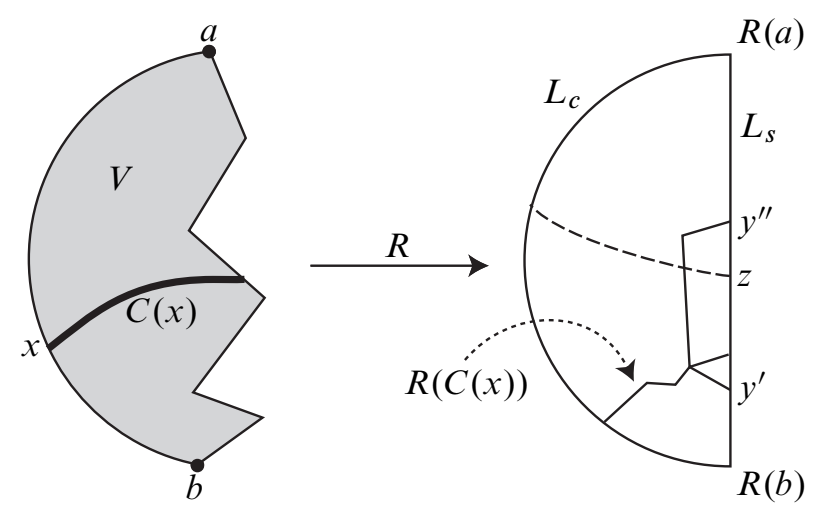

Figure 10.8.i: This picture illustrates the effect of the Riemann map $R$ in the proof of Proposition 10.7. It shows why the dashed line from $z$, which is supposed to be disjoint from $\overline{R(C(x))}$, cannot exist. The formal argument in the text is that the arc represented by the dashed line, joining $z$ to $L_{c}$ in the complement of $\overline{R(C(x))}$, would separate $y^{\prime \prime}$ from $x$, which is impossible.

The situation to be described below is illustrated in Figure 10.8.i. $C(x)$ is a connected subset of $V$, and therefore $R(C(x))$ is connected. It follows that the closure $\overline{R(C(x))}$ in $\mathbb{C}$ is connected. Let $Y(x)=\overline{R(C(x))} \cap L_{s} . Y(x)$ is a compact subset of $\mathbb{C}$. Since the closure of $C(x)$ meets $B(a, b)$, we see that $Y(x) \neq \varnothing$. It follows from Lemma 10.6 that, for each $x, f(C(x))=C(x)$. 
$V$ here plays the role of $U_{0}$ in the proof of Proposition 7.4. $R$ here plays the role of $\Phi$ there. The subspace $Y(x)$ here plays the role of $Y(r)$ there. In brief, we show that, for each $x, Y(x)$ is a singleton that is fixed by $f$. This shows that $f$ fixes $C$, and therefore its closure $N(a, b)$. This completes the proof of Proposition 10.7 and hence of Theorem 10.1.

\section{The affine group on an interval}

We now do the hard analysis necessary to complete the proof of Theorem 1.1.

We turn our attention to the affine group. We will investigate the effect of $\mathcal{E}$ on this group. We will be able to show (but only approximately so) that, if $a>0$ and $b \in \mathbb{R}$, then $\mathcal{E}$ must send the map $x \mapsto a x+b$ for $x \in \mathbb{R}$ to the map $z \mapsto a z+b$ for $z \in \mathbb{U}^{2}$. Of course, $\mathcal{E}$ can always be changed by conjugating by a quasiconformal homeomorphism of $\mathbb{U}^{2}$ that is the identity on the boundary. So the result is only proved up to such a conjugation. And even that is considerably more than what we really prove-nevertheless, this is the idea of the proof, and the idea guides what we actually do.

Our work on the affine group will provide the basis for the proof of Theorem 12.1. This result uses in its hypothesis a certain type of continuity of $\mathcal{E}$. We have already shown (Theorem 10.1) that, if $\mathcal{E}$ exists, it must be continuous in the required sense. All this information will then rule out the existence of $\mathcal{E}$ and prove Theorem 1.1.

The proof of Theorem 12.1 depends on Proposition 3.6. But that result applies only to groups of quasisymmetric homeomorphisms that fix at least three points. This can be achieved by using a diffeomorphism $\mathbb{R} \rightarrow(-1,1)$ to conjugate the affine group so that it is supported in a finite interval. The three points are chosen outside this interval. Such a conjugated affine map will in general fail to be quasisymmetric near the two endpoints of the interval. We need to choose our conjugating homeomorphism with care, so as to give a version of the affine group, supported in a finite interval, and acting by homeomorphisms that are quasisymmetric on the whole of $\mathbb{R} \cup\{\infty\}$. Even more difficult, in order for Proposition 3.6 to be applicable, we have to arrange for these quasisymmetric homeomorphisms to be near the identity in the Teichmüller metric.

In this section we will concentrate on producing the conjugation to a finite interval so that the conjugated homeomorphisms have the required properties. The proofs are analytical in the classical sense, and the homomorphism $\mathcal{E}$ does not make an appearance. 
11.1 Definition Given $a>0$ and $b \in \mathbb{R}$, we define the affine transformation $A_{a, b}: \mathbb{R} \rightarrow$ $\mathbb{R}$ by $A_{a, b}(x)=a x+b$. Let $A=\left\{A_{a, b}\right\}$ be the group of all orientation preserving affine transformations of the real line. We are also interested in the 1-parameter group of earthquake homeomorphisms $\left\{R_{a}\right\}_{a>0}$. Here $R_{a}: \mathbb{R} \rightarrow \mathbb{R}$ is defined so that $R_{a}(x)=x$ for $x<0$ and $R_{a}(x)=a x$ for $x \geq 0$.

Let $I$ be an open interval and let $\phi: \mathbb{R} \rightarrow I$ be a homeomorphism. We can make $A$ act on $I$ by conjugating with $\phi$. Since all elements of $A$ preserve orientation, we can extend the action of $A$ on $I$ to an action on $\mathbb{R}$ by making it act by the identity outside $I$. The same applies to the earthquake subgroup.

11.2 Theorem Let $\epsilon>0$. Then there are constants $L$ and $M$, with $M>L / \epsilon>1 / \epsilon^{2}$, and a $C^{1}$-diffeomorphism $\Psi_{\epsilon}: \mathbb{R} \rightarrow(-M, M)$ with the following properties:

(11.2.1) For $x \in \mathbb{R}, \Psi_{\epsilon}(-x)=-\Psi_{\epsilon}(x)$.

(11.2.2) $\Psi_{\epsilon} \mid[-L, L]$ is the identity.

(11.2.3) We recall the notation from Definition 11.1. We define $B_{a, b}: \mathbb{R} \rightarrow \mathbb{R}$ by setting it equal to $\Psi_{\epsilon} \circ A_{a, b} \circ \Psi_{\epsilon}^{-1}$ on $(-M, M)=\Psi_{\epsilon}(\mathbb{R})$ and equal to the identity elsewhere. Then $B_{a, b}$ is a quasisymmetric homeomorphism.

(11.2.4) If, further, $\epsilon<a<1 / \epsilon$ and $-1<\epsilon b<1$, then $d_{\mathcal{T}}\left(B_{a, b}\right.$, Id $)<\epsilon$.

(11.2.5) We recall the definition of the earthquake map $R_{a}: \mathbb{R} \rightarrow \mathbb{R}$ from Definition 11.1. Following the model introduced in Definition 11.1, we proceed as in the case of affine homeomorphisms, conjugating $R_{a}$ to $(-M, M)$ by using $\Psi_{\epsilon}$, and extending the conjugate to the rest of $\mathbb{R}$ by setting the extension to the identity outside $(-M, M)$. The resulting map $R_{a, \epsilon}$ is quasisymmetric. There is a continuous function $\beta:[0, \infty) \rightarrow[0, \infty)$, such that $\beta(0)=0$ and $d_{\mathcal{T}}\left(R_{a, \epsilon}, \mathrm{Id}\right) \leq \beta(|\log (a)|)$ for sufficiently small values of $\epsilon>0$. The function $\beta$ is defined in the proof of Lemma 11.4. Its definition is independent of $\epsilon$.

(11.2.6) There is a quasisymmetric homeomorphism $P_{\epsilon}: \mathbb{R} \rightarrow \mathbb{R}$ with compact support, such that $P_{\epsilon}(-M, M)=(-M, M)$ and $\Psi_{\epsilon}^{-1}\left(P_{\epsilon}\left(\Psi_{\epsilon}(t)\right)\right)=t^{2}$. The support of $P_{\epsilon}$ is equal to $[-M-1, M+1]$. It should be noted that this is larger than the range of $\Psi_{\epsilon}$, which is $(-M, M)$ and so the data here does not determine $P_{\epsilon}$ completely.

In our construction, the $B_{a, b}$ are $C^{\infty}$ except at $\pm L$ and $\pm M$. They are $C^{1}$ near $\pm L$ and, if $a \neq 1$, not even differentiable at $\pm M$. They are, however, quasisymmetric, as stated in (11.2.3). 
Proof We will choose $\delta>0$, depending on $\epsilon$, and then a diffeomorphism $\psi_{\delta}$ depending on $\delta . \Psi_{\epsilon}$ will be a linear rescaling of $\psi_{\delta}$.

11.3 Definition We assume $0<\delta$ and that $\delta$ is small. The diffeomorphism $\psi_{\delta}: \mathbb{R} \rightarrow$ $(-1,1)$ is defined in terms of another diffeomorphism $\alpha:(0, \infty) \rightarrow(0,1)$. The use of the notation $\alpha$ for this map is confined to this paragraph. We define $\alpha(t)=\exp \left(-t^{-\delta}\right)$. It is easy to see that $\alpha:(0, \infty) \rightarrow(0,1)$ is strictly monotonic and $C^{\infty}$. If we extend $\alpha$ by continuity to $\alpha:[0, \infty) \rightarrow[0,1)$, so that $\alpha(0)=0$, we find that all derivatives of $\alpha$ are 0 at 0 . There is exactly one point $t_{0}>0$, namely $t_{0}=\delta^{1 / \delta}$, such that $\alpha^{\prime}\left(t_{0}\right)=\alpha(t) / t$. We have $\alpha\left(t_{0}\right)=\exp (-1 / \delta)$ and we denote this point by $x_{0}$. The tangent line to the graph of $\alpha$ at $\left(t_{0}, \alpha\left(t_{0}\right)\right)$ goes through the origin. The graphs of $\alpha$ and $\psi_{\delta}$ are shown in Figure 11.3.i.

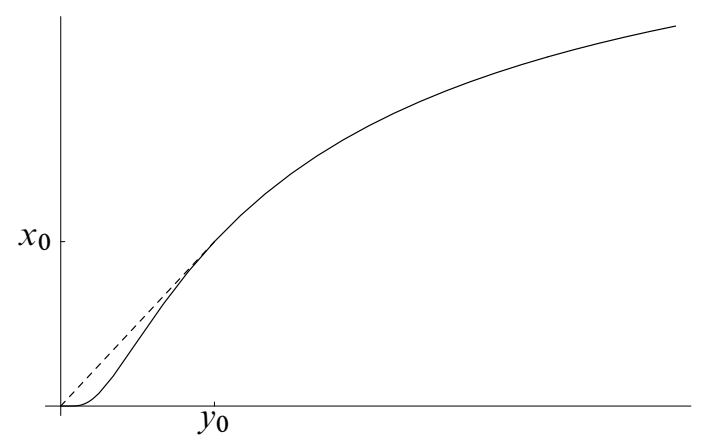

Figure 11.3.i: This picture illustrates the definition of $\psi_{\delta}$ (see Definition 11.3). The solid curve represents the graph of $\alpha$. The dotted curve shows how $\alpha$ has to be changed so that it becomes linear near 0 .

On $\left[-t_{0}, t_{0}\right]$ we set $\psi_{\delta}(t)=t x_{0} / t_{0}=t(e \delta)^{-1 / \delta}$, which is a linear map with very large slope for small $\delta$. (We will in due course take $\delta$ extremely small, and $t_{0}$ is very much smaller.) On $\left[t_{0}, \infty\right)$, we set $\psi_{\delta}(t)=\alpha(t)$. On $(-\infty, 0)$, we set $\psi_{\delta}(t)=-\psi_{\delta}(-t)$. The two definitions of $\psi_{\delta}$ on $\left[-t_{0}, 0\right)$ agree with each other. Then $\psi_{\delta}: \mathbb{R} \rightarrow(-1,1)$ is a surjective $C^{1}$-diffeomorphism.

Using the following lemma, we can check some of the assertions of quasisymmetry in Theorem 11.2.

11.4 Lemma There is a continuous function $\beta:[0, \infty) \rightarrow[0, \infty)$, such that $\beta(x)=0$ if and only if $x=0$, and such that $\beta$ has the following properties. Let $f:(0, \infty) \rightarrow$ $(0, \infty)$ and $g:(0, \infty) \rightarrow(0, \infty)$ be orientation preserving homeomorphisms, such that 
$f, g, f^{-1}$ and $g^{-1}$ are piecewise $C^{1}$-diffeomorphisms. Let $h: \mathbb{R} \rightarrow \mathbb{R}$ be defined by $h(0)=0$, and, if $t>0, h(t)=f(t)$ and $h(-t)=-g(t)$. Suppose that there is a $c>0$ such that, for all $t>0, f(t) / g(t), f^{\prime}(t) t / f(t)$ and $g^{\prime}(t) t / g(t)$ all lie in the interval $\left(e^{-c}, e^{c}\right)$. Then $h$ is quasisymmetric and $d_{\mathcal{T}}(h, \mathrm{Id})<\beta(c)$. In particular, if $c$ is small, then $d_{\mathcal{T}}(h, \mathrm{Id})$ is small.

Proof Let $S$ be a horizontal strip of height $\pi$ and let $\mathbb{U}^{2}$ be the upper halfplane. We transfer the problem to $\partial S$ and $S$ by conjugating with log: $\mathbb{U}^{2} \rightarrow S$ and exp: $S \rightarrow \mathbb{U}^{2}$. Let $u, v: \mathbb{R} \rightarrow \mathbb{R}$ be defined by $u(x)=\log (f(\exp (x)))$ and $v(x)=\log (g(\exp (x)))$. We define $F: S \rightarrow S$ by

$$
F(x+i y)=\frac{(\pi-y) u(x)+y v(x)}{\pi}+i y .
$$

Then, on $\mathbb{R}, h(t)=\exp (F(\log (t)))$, where we are using the usual complex form of $\log$, so that, if $t<0$, then $\log (t)=i \pi+\log (-t)$.

We then have

$$
\begin{aligned}
& 2 F_{z}=F_{x}-i F_{y}=(1-y / \pi) u^{\prime}(x)+(y / \pi) v^{\prime}(x)+1-i(v(x)-u(x)) / \pi \text { and } \\
& 2 F_{\bar{z}}=F_{x}+i F_{y}=(1-y / \pi) u^{\prime}(x)+(y / \pi) v^{\prime}(x)-1+i(v(x)-u(x)) / \pi .
\end{aligned}
$$

We will show that the absolute value of the Beltrami coefficient of $F$ (sometimes called its complex dilatation) has a supremum that is bounded away from 1 by a function of $c$. (See Section 13 for the definition of the Beltrami coefficient.) The square of the absolute value of the Beltrami coefficient is equal to

$$
\begin{aligned}
\left|\frac{F_{\bar{z}}}{F_{z}}\right|^{2} & =\left|\frac{(1-y / \pi) u^{\prime}+\left(y v^{\prime} / \pi\right)-1+i(v-u) / \pi}{(1-y / \pi) u^{\prime}+\left(y v^{\prime} / \pi\right)+1-i(v-u) / \pi}\right|^{2} \\
& =\frac{\left((1-y / \pi) u^{\prime}+\left(y v^{\prime} / \pi\right)-1\right)^{2}+((v-u) / \pi)^{2}}{\left((1-y / \pi) u^{\prime}+\left(y v^{\prime} / \pi\right)+1\right)^{2}+((v-u) / \pi)^{2}} .
\end{aligned}
$$

Now $v-u=\log \circ(f / g) \circ \exp$, and so $-c \leq|v-u| \leq c$. We have

$$
\begin{aligned}
u^{\prime}(x) & =f^{\prime}(\exp (x)) \exp (x) / f(\exp (x))=f^{\prime}(t) t / f(t) \text { and } \\
v^{\prime}(x) & =g^{\prime}(\exp (x)) \exp (x) / g(\exp (x))=g^{\prime}(t) t / g(t) .
\end{aligned}
$$

and so $u^{\prime}(x)$ and $v^{\prime}(x)$ both lie in the interval $\left(e^{-c}, e^{c}\right)$. The same therefore follows for the convex combination $(1-y / \pi) u^{\prime}(x)+(y / \pi) v^{\prime}(x)$.

To estimate the absolute value of the Beltrami coefficient, we consider the function $\mu: S \rightarrow[0,1)$ defined by

$$
\mu(r, s)^{2}=\frac{(r-1)^{2}+s^{2}}{(r+1)^{2}+s^{2}} .
$$


We are interested in bounding $\mu(r, s)$ above when $r=(1-y / \pi) u^{\prime}(x)+(y / \pi) v^{\prime}(x)$ and $s=(v-u) / \pi$. It is sufficient to bound $\mu(r, s)$ above, when $(r, s)$ is in the closed rectangle $R$ given by $e^{-c} \leq r \leq e^{c}$ and $0 \leq s \leq c / \pi$. By compactness, $\mu(r, s)$ achieves its maximum in $R$ at some point $\left(r_{0}, s_{0}\right) \in R$.

Since $\mu(r, s)$ is a strictly increasing function of $s$, it follows that $s_{0}=c / \pi$. We therefore have only to maximize $\mu\left(r, s_{0}\right)$ as a function of $r \in\left[e^{-c}, e^{c}\right]$. We have

$$
\frac{\partial \log \left(\mu\left(r, s_{0}\right)\right)}{\partial r}=\frac{4\left(r^{2}-s_{0}^{2}-1\right)}{\left((r-1)^{2}+s_{0}^{2}\right)\left((r+1)^{2}+s_{0}^{2}\right)} .
$$

This is zero at at most one point, namely $r=\sqrt{1+s_{0}^{2}}$ (which may or may not lie in the closed interval $\left[e^{-c}, e^{c}\right]$. For smaller values, $\mu\left(r, s_{0}\right)$ is a decreasing function of $r$, and for larger values, it is an increasing function of $r$. It follows that either $r_{0}=e^{-c}$ or $r_{0}=e^{c}$.

Finally, we see that the square of the absolute value of the Beltrami coefficient is bounded above by

$$
\begin{aligned}
& \max \left\{\frac{\left(e^{-c}-1\right)^{2}+(c / \pi)^{2}}{\left(e^{-c}+1\right)^{2}+(c / \pi)^{2}}, \frac{\left(e^{c}-1\right)^{2}+(c / \pi)^{2}}{\left(e^{c}+1\right)^{2}+(c / \pi)^{2}}\right\} \\
& \leq \gamma(c)=\frac{\left(e^{c}-1\right)^{2}+e^{2 c}(c / \pi)^{2}}{\left(e^{c}+1\right)^{2}+e^{2 c}(c / \pi)^{2}} \\
& <1 .
\end{aligned}
$$

The above equality serves to define $\gamma(c)$. We set

$$
\beta(c)=\log (1+\sqrt{\gamma(c)})-\log (1-\sqrt{\gamma(c)}) .
$$

This completes the proof.

11.5 Lemma Let $a>0$. Let $f=f_{\delta}: \mathbb{R} \rightarrow \mathbb{R}$ be the homeomorphism which is the identity outside $(-1,1)$ and is defined by $f(x)=\psi_{\delta}\left(a \psi_{\delta}^{-1}(x)\right)$ for $-1<x<1$. Then $f$ is quasisymmetric and

$$
d_{\mathcal{T}}(f, \mathrm{Id}) \leq \beta(\delta|\log (a)|) .
$$

Suppose $\epsilon \leq a \leq 1 / \epsilon$. If $\delta<\epsilon /|\log (\epsilon)|$, then $d_{\mathcal{T}}(f$, Id $)<\beta(\epsilon)$. Also, $f$ is $C^{1}$ except at \pm 1 . The limit of $f^{\prime}(x)$ as $x$ tends to 1 from below is $a^{-\delta}$, and this is also equal to the left derivative of $f$ at 1 . 
Proof According to Lemma 11.4, we need to estimate $\log \left(f^{\prime}(x) x / f(x)\right)$ for $x>0$. If $x>1$, this is equal to zero. For $0<x<1$, we define $t$ by $\psi_{\delta}(t)=x$. Then $f^{\prime}(x)=\psi_{\delta}^{\prime}(a t) a / \psi_{\delta}^{\prime}(t)$ and $f(x)=\psi_{\delta}(a t)$. We set

$$
q(t)=\frac{\psi_{\delta}^{\prime}(a t)}{\psi_{\delta}(a t)} \cdot \frac{\psi_{\delta}(t)}{\psi_{\delta}^{\prime}(t)} \cdot a=\frac{f^{\prime}(x) x}{f(x)} .
$$

Then $d_{\mathcal{T}}(f$, Id $) \leq \beta\left(\sup _{t} \mid \log (q(t))\right) \mid$, where $\beta$ is the function that came up in Lemma 11.4. So we need to bound $|\log (q(t))|$. Since $d_{\mathcal{T}}(f, \mathrm{Id})=d_{\mathcal{T}}\left(f^{-1}\right.$, Id $)$, we may as well take $a>1$. Note that $\psi_{\delta}^{\prime}(t) / \psi_{\delta}(t)=\delta t^{-\delta-1}$ for $t \geq t_{0}$.

The first case is $t<a t \leq t_{0}$, where $t_{0}$ comes from Definition 11.3. In this range, $\psi_{\delta}$ is linear, $q(t)=1$ and $\log (q(t))=0$. The second case is $t_{0} \leq t<a t$. Then $q(t)=a^{-\delta}$ and $|\log (q(t))|=\delta \log (a)$. The third case is $t<t_{0}=\delta^{1 / \delta}<a t$, which implies that

$$
t^{-\delta}>t_{0}^{-\delta}=1 / \delta>(a t)^{-\delta} .
$$

In this case, $\psi_{\delta}(t) / \psi_{\delta}^{\prime}(t)=t$, since $\psi_{\delta}$ is linear on $[0, t]$. So

$$
a^{-\delta}<q(t)=\delta a^{-\delta} t^{-\delta}<1 .
$$

It follows that $|\log (q(t))|<\delta \log (a)$.

The computation in the second case shows that $f^{\prime}(x) x / f(x)$ is equal to $a^{-\delta}$ for $x_{0}=\psi_{\delta}\left(t_{0}\right)<x<1$. When $x$ tends to 1 , so does $f(x)$. It follows that $f^{\prime}(x) \approx a^{-\delta}$ for $x<1$ and $x$ near 1 . The First Mean Value Theorem shows that $f$ is differentiable on the left at 1 , with $f_{-}^{\prime}(1)=a^{-\delta}$. Since $f^{\prime}(x)=1$ for $x>1$, there is a discontinuity in $f^{\prime}$ at $x=1$, and similarly for $x=-1$.

11.6 Lemma Let $b \in \mathbb{R}$. Let $f_{b}=f_{b, \delta}: \mathbb{R} \rightarrow \mathbb{R}$ be the homeomorphism which is the identity outside $(-1,1)$ and is defined by $f_{b}(x)=\psi_{\delta}\left(b+\psi_{\delta}^{-1}(x)\right)$ for $-1<x<1$. Then $f_{b}$ is $C^{1}$ and therefore quasisymmetric. For $|b|$ sufficiently small, $d_{\mathcal{T}}\left(f_{b}, \mathrm{Id}\right)<$ $\epsilon / 2$.

Proof For $-1<x<1, f_{b}$ is the composition of $C^{1}$-functions and is therefore $C^{1}$. It is also $C^{1}$ for $|x|>1$. By symmetry, to prove that $f_{b}$ is $C^{1}$ everywhere, it only remains to check near $x=1$. For $x<1$, we write $x=1-u$, with $u$ small. Using $\psi_{\delta}^{-1}(x)=(-\log (x))^{-1 / \delta}$, we expand $f_{b}(1-u)$ in terms of $u>0$. A careful calculation shows that both left and right derivatives of $f_{b}$ at $x=1$ are equal to 1 , and that the derivative is continuous at $x=1$. Since $f_{b}$ is $C^{1}$, and is supported on $[-1,1]$, it is also quasisymmetric. 
To understand the dependence of $f_{b}$ on $b$, we need to understand the function $\phi$ defined by

$$
\phi(x, b)=f_{b}(x)=\psi_{\delta}\left(b+\psi_{\delta}^{-1}(x)\right)
$$

when $-1<x<1$ and by $\phi(x, b)=x$ when $|x| \geq 1$. We will prove that the derivative $\partial \phi(x, b) / \partial x$ tends uniformly to 1 as $b$ tends to 0 . We may assume that $-1<x<1$. Since $f_{-b}=f_{b}^{-1}$, we may assume that $b>0$.

We have $\partial \phi(x, b) / \partial x=\psi_{\delta}^{\prime}(t+b) / \psi_{\delta}^{\prime}(t)$, where $\psi_{\delta}(t)=x$. The first case is $-t_{0} \leq$ $t<t+b \leq t_{0}$. Then $\psi_{\delta}$ is linear and $\partial \phi(x, b) / \partial x=1$.

The second case is $t_{0} \leq t$. Then the partial derivative is

$$
\exp \left(\frac{1}{t^{\delta}}-\frac{1}{(t+b)^{\delta}}\right) \cdot \frac{t^{1+\delta}}{(t+b)^{1+\delta}} .
$$

The second factor tends uniformly to 1 on $\left[t_{0}, \infty\right)$ as $b$ tends to 0 . Considering the first factor, we see that, for $t_{0} \leq t$,

$$
0<t^{-\delta}-(t+b)^{-\delta} \leq t_{0}^{-\delta}\left(1-\left(1+b / t_{0}\right)^{-\delta}\right) .
$$

The righthand side of this inequality tends uniformly to 0 as $b$ tends to 0 and its exponential tends to 1 .

The third case is $t<t_{0}<t+b$. The partial derivative is then

$$
\delta \exp \left(-(t+b)^{-\delta}\right) t^{-(1+\delta)}(e \delta)^{1 / \delta} .
$$

We may rewrite this as

$$
\left(t_{0} / t\right)^{1+\delta} \exp \left(\frac{1}{\delta}-(t+b)^{-\delta}\right)=\left(t_{0} / t\right)^{1+\delta} \exp \left(t_{0}^{-\delta}-(t+b)^{-\delta}\right) .
$$

In this case, $t_{0} / t$ tends to 1 and $t_{0}^{-\delta}-(t+b)^{-\delta}$ tends to 0 uniformly as $b$ tends to 0 . So the partial derivative tends uniformly to 1 as $b$ tends to zero.

From the Mean Value Theorem, we can now deduce that

$$
\frac{f_{b}(x+h)-f_{b}(x)}{f_{b}(x)-f_{b}(x-h)}
$$

converges to 1 uniformly as $b$ tends to 0 . By Paragraph 13.4, this proves that the Teichmüller distance from the identity tends to zero.

11.7 Lemma Let $a>0$, and let $f=f_{\delta}$ be the homeomorphism obtained by conjugating the earthquake map $R_{a}$ on $\mathbb{R}$ to $(-1,1)$, using $\psi_{\delta}$. Explicitly, $f: \mathbb{R} \rightarrow \mathbb{R}$ is the homeomorphism which is the identity outside $(0,1)$ and is defined by $f(x)=$ 
$\psi_{\delta}\left(a \psi_{\delta}^{-1}(x)\right)$ for $0<x<1$. Then $f$ is quasisymmetric. If $\delta<1$, then $d_{\mathcal{T}}(f$, Id $) \leq$ $\beta(|\log (a)|)$, where $\beta$ is the function of Lemma 11.4.

Proof We will prove this result by applying Lemma 11.4. First we have to find a bound for $\log \left(f^{\prime}(x) x / f(x)\right)$. Outside $(0,1)$, this quantity is equal to 0 . In $(0,1)$, we have already found an explicit bound in the proof of Lemma 11.5, namely $\delta|\log (a)|$.

We also have to find a bound for $\log (f(x) / x)$. For $x$ outside $(0,1)$, this quantity is equal to 0 , so we may assume that $0<x<1$. Defining $t$ by $\psi_{\delta}(t)=x$, we have to bound $\log \left(\psi_{\delta}(a t) / \psi_{\delta}(t)\right)$, which we denote by $\lambda(t)$. We may assume without loss of generality that $a>1$. If $t<a t \leq t_{0}=\delta^{1 / \delta}$, then $\lambda(t)=\log (a)$.

Now we assume that $t_{0} \leq t<a t$. Then

$$
\lambda(t)=-(a t)^{-\delta}+t^{-\delta}=\frac{1-a^{-\delta}}{t^{\delta}} .
$$

For all $t \geq t_{0}, \lambda(t)>0$ and $\lambda(t)$ decreases as $t$ increases. The maximum value occurs at $t=t_{0}$, and $\lambda\left(t_{0}\right)=\left(1-a^{-\delta}\right) / \delta$. This is a continuous function of $\delta$. By calculus, it increases as $\delta$ decreases, and the limit as $\delta$ tends to zero is $\log (a)$. This gives $\lambda(t) \leq \log (a)$.

Finally, we have to estimate $\psi_{\delta}(a t) / \psi_{\delta}(t)$ when $t<t_{0}<a t$. In this case we have

$$
\frac{\psi_{\delta}(a t)}{\psi_{\delta}(t)}=\frac{\psi_{\delta}(a t)}{t \psi_{\delta}^{\prime}\left(t_{0}\right)}
$$

We have $\psi_{\delta}^{\prime}\left(t_{0}\right)=(e \delta)^{-1 / \delta}$. Therefore

$$
\lambda(t)=-a^{-\delta} t^{-\delta}-\log (t)+1 / \delta+\log (\delta) / \delta .
$$

We want to find the maximum value of $\lambda(t)$ for $t \in\left[t_{0} / a, t_{0}\right]$. Differentiating, we find

$$
\lambda^{\prime}(t)=\delta a^{-\delta} t^{-\delta-1}-1 / t .
$$

The sign of $\lambda^{\prime}(t)$ is equal to the sign of $\delta a^{-\delta}-t^{\delta}$, which decreases as $t$ increases. For $t=t_{0} / a$, this quantity is zero. It follows that $\lambda^{\prime}(t)<0$ for $t \in\left(t_{0} / a, t_{0}\right]$. So the maximum value of $\lambda(t)$ for $t \in\left[t_{0} / a, t_{0}\right]$ is $\lambda\left(t_{0} / a\right)=\log (a)$.

In the notation of Lemma 11.4, we have shown that $d_{\mathcal{T}}(f, \mathrm{Id}) \leq \beta(|\log (a)|)$. This completes the proof of Lemma 11.7.

In order to prove (11.2.6), we first prove a lemma which could be pushed much further; but the present version is easy to deal with and is sufficient in our situation. 
11.8 Lemma Let $x_{0}<x_{1}<\ldots<x_{n}$ be a finite increasing sequence of real numbers. For each $i$ with $0<i<n$, let $c_{i} \neq 0$ be a real number, and let $r_{i}$ and $s_{i}$ be real analytic functions of a real variable near 0 , with vanishing 2-jets at 0 . Let $f: \mathbb{R} \rightarrow \mathbb{R}$ be a homeomorphism. Then $f$ is quasisymmetric if the following conditions are satisfied.

(11.8.1) The restriction of $f$ to $\left(-\infty, x_{0}\right] \cup\left[x_{n}, \infty\right)$ is the identity.

(11.8.2) The restriction of $f$ to $\left(-\infty, x_{1}\right) \cup\left(x_{n-1}, \infty\right)$ is $C^{1}$ with strictly positive derivative.

(11.8.3) For each $i$ with $1<i<n$ the restriction $f \mid\left(x_{i-1}, x_{i}\right)$ is $C^{1}$, with strictly positive derivative.

(11.8.4) Suppose that $1<i<n, x \geq x_{i}$ and that $x$ is near $x_{i}$. Then

$$
f(x)=f\left(x_{i}\right)+c_{i}\left(\left(x-x_{i}\right)^{2}+r_{i}\left(x-x_{i}\right)\right) .
$$

(This is a condition on $f$, not a result to be proved.)

(11.8.5) Suppose that $1<i<n, x \leq x_{i}$ and that $x$ is near $x_{i}$. Then

$$
f(x)=f\left(x_{i}\right)-c_{i}\left(\left(x-x_{i}\right)^{2}+s_{i}\left(x-x_{i}\right)\right) .
$$

(Another condition on $f$.)

Proof We will suppose that $f$ is not quasisymmetric and deduce a contradiction. Let $\left(u_{j}\right)_{j \in \mathbb{N}}$ be a sequence of real numbers and let $\left(h_{j}\right)_{j \in \mathbb{N}}$ be a sequence of strictly positive real numbers such that

$$
\frac{f\left(u_{j}+h_{j}\right)-f\left(u_{j}\right)}{f\left(u_{j}\right)-f\left(u_{j}-h_{j}\right)}
$$

converges either to infinity or to zero. See Paragraph 13.4 for references about quasisymmetry. We may assume that $\left(u_{j}\right)_{j \in \mathbb{N}}$ converges to $+\infty$, to $-\infty$ or to a finite limit and that $\left(h_{j}\right)_{j \in \mathbb{N}}$ converges to $+\infty$, to a finite positive limit or to 0 . It is easy to see that the limit of $\left(u_{j}\right)_{j \in \mathbb{N}}$ cannot be infinite. Let $u_{\infty}$ be the finite limit. It is easy to see that the limit of $\left(h_{j}\right)_{j \in \mathbb{N}}$ cannot be infinite, and then that it must be zero. It follows that $x_{0} \leq u_{\infty} \leq x_{n}$ and that $f$ cannot be a $C^{1}$-diffeomorphism near $u_{\infty}$. It follows that $u_{\infty}=x_{i}$ for some $i$ with $0<i<n$. From now on $i$ is fixed in this proof. We change coordinates so that $u_{\infty}=x_{i}=0$.

We can assume that, for each $j, u_{j} \geq 0$. We may further assume that, for all $j$, $0<h_{j} \leq u_{j}$, or that, for all $j, 0 \leq u_{j}<h_{j}$. In the first case, we may assume that $h_{j} / u_{j}$ converges to a limit $\lambda_{1}$ with $0 \leq \lambda_{1} \leq 1$. In the second case, we may assume 
that $u_{j} / h_{j}$ converges to a limit $\lambda_{2}$ with $0 \leq \lambda_{2} \leq 1$. In order to have a less cluttered notation, we drop the subscript $j$.

In the first case, we have $0<h \leq u$ and we have to consider

$$
\frac{(u+h)^{2}-u^{2}+r(u+h)-r(u)}{u^{2}-(u-h)^{2}+r(u)-r(u-h)} .
$$

Now $(u+h)^{n}-u^{n}$ and $u^{n}-(u-h)^{n}$ are both polynomials in $u$ and $h$ which are divisible by $h$. Using the expression of $r$ as a power series starting with a cubic term, we see that we can divide both numerator and denominator in our expression by $h$. Our ratio is equal to $(2 u+h+o(u)) /(2 u-h+o(u))$, which converges to $\left(2+\lambda_{1}\right) /\left(2-\lambda_{1}\right)$. But we are assuming that the limit is either 0 or $\infty$, so this is a contradiction.

In the second case, we have $0 \leq u<h$ and we consider

$$
\frac{(u+h)^{2}-u^{2}+r(u+h)-r(u)}{u^{2}+(u-h)^{2}+r(u)+s(u-h)} .
$$

Dividing both numerator and denominator by $h^{2}$ and taking the limit, we obtain $\left(2 \lambda_{2}+1\right) /\left(\lambda_{2}^{2}+\left(1-\lambda_{2}\right)^{2}\right)$. This is also a contradiction, so Lemma 11.8 is proved.

11.9 Lemma There is a quasisymmetric homeomorphism $f=f_{\delta}: \mathbb{R} \rightarrow \mathbb{R}$ with compact support, such that $f(1)=1, f(-1)=-1$, and, for all $t \in \mathbb{R}, \psi_{\delta}^{-1} \circ f \circ \psi_{\delta}(t)=$ $\pm t^{2}$, where the sign is the same as that of $t$. The support of $f$ strictly contains $[-1,1]$.

Proof The statement of Lemma 11.9 forces the definition of $f \mid(-1,1)$. For $x<1$ and $x$ near 1 , we have

$$
f(x)=\exp \left(-\left(\left((-\log (x))^{-2 / \delta}\right)^{-\delta}\right)\right)=\exp \left(-(\log (x))^{2}\right) .
$$

We introduce a local coordinate $u=1-x$ near $x=1$. The image of $u$ is

$$
f(1-u)=\exp \left(-u^{2}+\ldots\right)=1-u^{2}+\gamma(u),
$$

where $\gamma$ is an analytic function with a zero 2-jet at 0 . In terms of local coordinates, $u$ is sent to $-u^{2}+\gamma(u)$. The situation for $x>-1$ and $x$ near -1 is similar. Using Lemma 11.8, we are therefore able to construct $f$ on a larger domain so that it is quasisymmetric.

We are now in a position to complete the proof of Theorem 11.2. Given $\epsilon>0$, we choose $\delta$ sufficiently small so that various Teichmüller distances are appropriately 
small. Explicitly, we use Lemma 11.5 to ensure that the conjugate (called $f$ in Lemma 11.5) of $x \mapsto a x$ is at a Teichmüller distance less than $\epsilon / 2$ from 0 if $\epsilon<a<1 / \epsilon$. By Lemma 11.7, this ensures that the conjugate $f$ of the earthquake $R_{a}$ satisfies $d_{\mathcal{T}}(f, \mathrm{Id}) \leq \beta(|\log (a)|)$, which is independent of $\epsilon$ and $\delta$. We define

$$
\Psi_{\epsilon}(t)=\frac{N_{\epsilon}}{x_{0}} \psi_{\delta}\left(\frac{t_{0} t}{N_{\epsilon}}\right)
$$

where $N_{\epsilon}>0$ will be chosen shortly. Then $\Psi_{\epsilon}: \mathbb{R} \rightarrow\left(-N_{\epsilon} / x_{0}, N_{\epsilon} / x_{0}\right)$ is a $C^{1}-$ diffeomorphism and $\Psi_{\epsilon}(t)=t$ if $|t| \leq N_{\epsilon}$.

To choose $N_{\epsilon}$, we refer to Lemma 11.6. This states that there is an $\epsilon_{1}>0$, such that the conjugate $f$ of $t \mapsto t+b$ satisfies $d_{\mathcal{T}}(f$, Id $)<\epsilon / 2$ if $|b|<\epsilon_{1}$. Notice that scaling in the domain or range does not change dilatations or Teichmüller distances, and we are therefore free to choose $N_{\epsilon}$ as large as we wish. We choose $N_{\epsilon}$ large enough so that $t_{0}<N_{\epsilon} \epsilon \epsilon_{1}$. If $|b| \leq 1 / \epsilon$, then $t_{0}|b| / N_{\epsilon}<\epsilon_{1}$. It follows that the map $g_{b}$, given by $x \mapsto \Psi_{\epsilon}\left(b+\Psi_{\epsilon}^{-1}(x)\right)$ for $|x|<N_{\epsilon} / x_{0}$ and otherwise by $x \mapsto x$, satisfies $d_{\mathcal{T}}\left(g_{b}\right.$, Id $)<\epsilon / 2$.

This deals with all parts of Theorem 11.2 except for (11.2.6), an analogue of which was proved in Lemma 11.9. The difference is that in (11.2.6) one uses $\Psi_{\epsilon}$, whereas in Lemma 11.9 one uses $\psi_{\delta}$. These differ only through multiplication by two constants, one in the domain and the other in the range. We now repeat the proof of Lemma 11.9, noting that each of the two constants must initially appear twice in the formulas, once for $\Psi_{\epsilon}$ and once for $\Psi_{\epsilon}^{-1}$. Lemma 11.8 is used in the same way that it was used in the proof of Lemma 11.9.

This completes the proof of Theorem 11.2.

11.10 Note In the above proof it is sufficient to take

$$
N_{\epsilon}>t_{0} /\left(\epsilon \epsilon_{1}\right) \text {. }
$$

We have not determined $\epsilon_{1}$ explicitly in terms of $\epsilon$ and so we are not entitled to assume that it increases with $\epsilon$. Note also that the map $P_{\epsilon}$ of (11.2.6) does not have bounded quasisymmetric constants as $\epsilon$ tends to zero. As a result, special arguments will become necessary when taking limits of maps like $P_{\epsilon}$.

\section{Continuous extensions}

In this section we complete the proof of Theorem 1.1, stating that the extension homomorphism $\mathcal{E}$ does not exist. We are assuming it does exist, and we are busy showing that this leads to a contradiction. 
We recall the definition of $F(x)$, for $x \in \mathbb{S}^{1}$, given in Definition 8.2. There are two cases: $F(x) \neq\{x\}$ and $F(x)=\{x\}$. The first case was shown to be impossible in Section 9. The second case has as its consequence (see Theorem 10.1) a certain type of continuity for $\mathcal{E}$. We now restate this condition, and show that it leads to a contradiction, thus completing the proof of Theorem 1.1.

12.1 Theorem It is not possible to find a homomorphism $\mathcal{E}: \mathcal{Q S} \rightarrow Q \mathcal{C}$ and an open $\mathcal{E}(Q \mathcal{S})$-invariant neighbourhood $U$ of $\mathbb{S}^{1}=\partial D^{2}$ in $\mathbb{D}^{2}$ with the following two properties:

(12.1.1) The composite

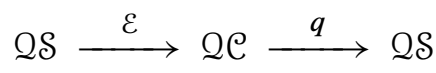

is the identity.

(12.1.2) Let $\left(u_{n}\right)_{n \in \mathbb{N}}$ be a sequence in $Q \mathcal{S}$, converging uniformly to some $u \in$ QS. Suppose further that the sequence $\left(\mathcal{E}\left(u_{n}\right)\right)_{n \in \mathbb{N}}$ has uniformly bounded dilatation. Then the sequence $\left(\mathcal{E}\left(u_{n}\right)\right)_{n \in \mathbb{N}}$ converges uniformly to $\mathcal{E}(u)$ on compact subsets of $U$.

Under the hypotheses of Theorem 12.1, we can prove a more convenient form of Proposition 3.6.

12.2 Proposition Let $\mathcal{E}: \mathcal{Q S} \rightarrow$ QC satisfy the hypotheses of Theorem 12.1. Then, for each $r>0$, there is a $k>1$ such that, if $g \in \mathcal{Q S}_{3}$ and $d \mathcal{T}(g, \mathrm{Id}) \leq r$, then $\mathcal{E}(g) \mid U$ has dilatation bounded by $k$.

Proof First note that, under our continuity hypotheses, we may replace $Q$ in Proposition 3.6 by the entire $\epsilon_{0}$-ball centred at the identity in $\mathcal{Q S}$, provided we are trying to bound only the dilatation of quasiconformal homeomorphisms of the form $\varepsilon(g) \mid U$. The bound is $\exp \left(k_{0}\right)$, where $k_{0}$ comes from the statement of Proposition 3.6.

Let $f \in \mathcal{Q C}_{3}$ with $d_{\mathcal{T}}(f, \mathrm{Id}) \leq r$. In [1, page 99], Ahlfors shows how to factorize $f=f_{1} \ldots f_{m}$, where $f_{i} \in \mathcal{Q} \mathcal{C}_{3}$ and $d_{\mathcal{T}}\left(f_{i}\right.$, Id $) \leq r / m$. See Paragraph 13.5.

Now let $g \in \mathcal{Q S}_{3}$ and extend $g$ to $f \in Q C_{3}$, with $d_{\mathcal{T}}(g, \mathrm{Id})=d_{\mathcal{T}}(f$, Id $) \leq r$. Since $f$ can be factorized, so can $g$. We take an integer $m>r / \epsilon_{0}$ and write $g=g_{1} \ldots g_{m}$ with $d_{\mathcal{T}}\left(g_{i}, \mathrm{Id}\right) \leq r / m<\epsilon_{0}$. It follows that, for each $i$, the maximal dilatation of $\mathcal{E}\left(g_{i}\right) \mid U$ is bounded by $\exp \left(k_{0}\right)$. Therefore the maximal dilatation of $\mathcal{E}(g) \mid U$ is bounded by $\exp \left(m k_{0}\right)$, as required. 


\section{Proof of Theorem 12.1}

Later on we will choose a decreasing sequence of small positive numbers $(\epsilon(n))_{n \in \mathbb{N}}$. For the moment, let us suppose that such a sequence is given. We apply Theorem 11.2 with $\epsilon=\epsilon(n)$ together with Note 11.10 to choose strictly increasing sequences of constants $L_{n}>n$ and $M_{n}>n L_{n}$, and a $C^{1}$-diffeomorphism $\Psi_{\epsilon(n)}: \mathbb{R} \rightarrow\left(-M_{n}, M_{n}\right)$, such that $\Psi_{\epsilon(n)}$ is the identity map on $\left[-L_{n}, L_{n}\right]$.

12.3 Definition Given an orientation preserving homeomorphism map $h: \mathbb{R} \rightarrow \mathbb{R}$, we define a homomorphism $\Gamma_{n}(h)$ : $\operatorname{Homeo}(\mathbb{R}) \rightarrow \operatorname{Homeo}(\mathbb{R})$ by

$$
\Gamma_{n}(h)(x)= \begin{cases}\Psi_{\epsilon(n)}\left(h\left(\Psi_{\epsilon(n)}^{-1}(x)\right)\right) & \text { for }-M_{n}<x<M_{n} \\ x & \text { for }|x| \geq M_{n} .\end{cases}
$$

Let $G_{n} \subset Q \mathcal{S}$ be the subgroup of $h$ such that $\Gamma_{n}(h)$ is quasisymmetric. Let $G_{b} \subset \bigcap_{n} G_{n}$ be the subgroup consisting of those $h$ for which $d_{\mathcal{T}}\left(\Gamma_{n}(h), \mathrm{Id}\right)$ is bounded as $n$ varies. From now on, we will restrict the use of $\Gamma_{n}$ to mean the homomorphism $\Gamma_{n}: G_{b} \rightarrow Q \mathcal{S}$.

12.4 Lemma $G_{b}$ contains the affine group, the earthquake group, and any quasisymmetric homeomorphism with compact support. If $g \in G_{b},-L_{n} \leq x \leq L_{n}$ and $-L_{n} \leq g(x) \leq L_{n}$, then $\Gamma_{n}(g)(x)=g(x)$.

Proof This is an immediate consequence of Theorem 11.2

We will want to work with the squaring map $t \mapsto t^{2}$ as though it is in $G_{b}$. Unfortunately it is not in $G_{b}$, nor in any $G_{n}$. We will deal with this in due course, using a special trick一see Lemma 12.14 .

We work in the upper halfplane rather than the disk. $U$, previously a neighbourhood of $\mathbb{S}^{1}$ in Theorem 12.1, now becomes an open neighbourhood of $\mathbb{R} \cup\{\infty\}$. We denote by $\mathcal{Q S}_{3}$ the group of quasisymmetric homeomorphisms of $\mathbb{R}$ that fix 0,1 and $\infty$, and by $\mathrm{QC}_{3}$ the group of quasiconformal homeomorphisms of $\mathbb{U}^{2} \cup \mathbb{R}$ fixing the same three points. We apply Proposition 12.2 to show that, if we bound the Teichmüller distance of $g \in \mathcal{Q S}_{3}$ from the identity, then the Teichmüller distance of $\mathcal{E}(g) \in \mathcal{Q C}_{3}$ from the identity is also bounded.

In order to apply this result to the image of $\Gamma_{n}$, we need to move to the side the support of the image subgroup so that 0,1 and $\infty$ are fixed by all elements of $\Gamma_{n}\left(G_{b}\right)$. We therefore need to apply a translation $\tau_{n}$. In order to take care of our subsequent trick for the squaring map, we will need to translate by a little more than currently seems necessary. 
12.5 Definition We define $\tau_{n}$ to be the translation of $\mathbb{R}$ by $M_{n}+4$. We define $T_{n}$ to be translation of the upper halfplane by $M_{n}+4$.

Given any group $G$ and $g \in G$, we denote by $c(g): G \rightarrow G$ the inner automorphism defined by $c(g)\left(g_{1}\right)=g g_{1} g^{-1}$. Consider the homomorphism $\mathcal{E}_{n}: G_{b} \rightarrow$ QC defined by the composition

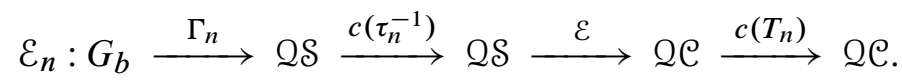

This is the same as

$$
\mathcal{E}_{n}(g)=T_{n} \mathcal{E}\left(\tau_{n}^{-1} \Gamma_{n}(g) \tau_{n}\right) T_{n}^{-1} .
$$

Notice that, for all $g \in G_{b}, \tau_{n}^{-1} \Gamma_{n}(g) \tau_{n}$ has support in $(-\infty, 0)$. Composition with $\tau_{n}$ or its inverse makes no difference to the Teichmüller distance from the identity in QS. It follows from Proposition 12.2 that the dilatation of $\mathcal{E}_{n}(g) \mid T_{n}(U)$ is bounded by a number depending only on the Teichmüller distance of $\Gamma_{n}(g)$ from the identity. Moreover, $\mathcal{E}_{n}(g) \mid \mathbb{R}=\Gamma_{n}(g)$ and, by Lemma $12.4, \Gamma_{n}(g)$ is equal to $g$ on $\left[-L_{n}, L_{n}\right] \cap$ $g^{-1}\left[-L_{n}, L_{n}\right]$. It follows that, for each $g \in G_{b}, \mathcal{E}_{n}(g) \mid \mathbb{R}$ converges to $g$ as $n$ tends to infinity, uniformly on compact subsets of $\mathbb{R}$. (In fact, for a given compact subset of $\mathbb{R}$, and a given $g$, the restriction of $\mathcal{E}_{n}(g)$ to the compact set is independent of $n$, provided $n$ is large enough.)

For fixed $g \in G_{b}$, we want convergence of $\mathcal{E}_{n}(g)$ as $n$ tends to infinity; at the moment, we only know that $\varepsilon_{n}(g) \mid \mathbb{R}$ converges to $g$. Given any compact subset $K \subset \mathbb{U}^{2}$, we have $K \subset T_{n}(U)$ for $n$ sufficiently large. Also, the dilatation of $\mathcal{E}_{n}(g) \mid T_{n}(U)$ is bounded in terms of the Teichmüller distance of $\Gamma_{n}(g)$ from the identity. It follows that, for each $g \in G_{b}$, there is a sequence $(n(i))_{i \in \mathbb{N}}$ for which $\left(\mathcal{E}_{n(i)}(g)\right)_{i \in \mathbb{N}}$ converges uniformly on compact subsets of $\mathbb{U}^{2}$. Since the restriction to $\mathbb{R}$ converges to $g$, the sequence must converge uniformly on compact subsets to a quasiconformal homeomorphism (and not to a constant map or to infinity)—see Section 13.

12.6 Definition The standard diagonalization argument now shows that, given any sequence $\left(g_{m}\right)_{m \in \mathbb{N}}$ of elements of $G_{b}$, we may assume that, for each $m$, the sequence $\left(\mathcal{E}_{n(i)}\left(g_{m}\right)\right)_{i \in \mathbb{N}}$ converges to a quasiconformal homeomorphism-we denote this limit by $\widehat{\varepsilon}\left(g_{m}\right): \mathbb{U}^{2} \rightarrow \mathbb{U}^{2}$.

We choose sequences in $G_{b}$ that help with our problem. Note that the definition of $\widehat{\mathcal{E}}$ depends on the choice of subsequence. We can always take a subsequence of the subsequence so that a further finite (or even countable) subset of elements of $G_{b}$ is incorporated into the domain of $\widehat{\mathcal{\varepsilon}}$. Note that, for all $g \in G_{b}, \widehat{\varepsilon}(g) \mid \mathbb{R}=g$. 
The definition of $\widehat{\mathcal{E}}$ will, in due course, be altered by a conjugation in Definition 12.7. The conjugating element will be the identity on $\mathbb{R}$, so that we will continue to have $\widehat{\mathcal{E}}(g) \mid \mathbb{R}=g$. We will also further enlarge the domain of our current definition of $\widehat{\mathcal{E}}$ by going to subsequences a fixed finite number of times.

Let $\left(A_{n}\right)_{n \in \mathbb{N}}$ be a sequence of affine homeomorphisms which is dense in the affine group, and let $\left(R_{n}\right)_{n \in \mathbb{N}}$ be a sequence of earthquakes which is dense in the earthquake group. We choose the $A_{n}$ and $R_{n}$ so that the set of all $A_{n}$ is a subgroup of $Q \mathcal{S}$, and similarly for the $R_{n}$. In fact, it is convenient to go further. We fix an irrational $\alpha$. We choose the first few $A_{n}$ so that $A_{1}(t)=t+1, A_{2}(t)=t+\alpha, A_{3}(t)=2 t$ and $A_{4}(t)=2^{\alpha} t$. Then $A_{1}$ and $A_{2}$ generate a subgroup that is dense in the subgroup of translations, and $A_{3}$ and $A_{4}$ generate a subgroup that is dense in the subgroup of all positive multiplications. $\left(A_{n}\right)_{n \in \mathbb{N}}$ is a listing of all the elements in the group generated by these four elements. For the group $\left(R_{n}\right)_{n \in \mathbb{N}}$, we use the group generated by $R_{1}$ and $R_{2}$ defined by $R_{1}(t)=2 t$ and $R_{2}(t)=2^{\alpha} t$ for $t \geq 0$ and by $R_{1}(t)=R_{2}(t)=t$ for $t \leq 0$. For each $n \in \mathbb{N}$, we define the constants $a_{n}$ and $b_{n}$ so that $A_{n}(t)=a_{n} t+b_{n}$.

We can now determine the sequence $(\epsilon(n))_{n \in \mathbb{N}}$. We choose $\epsilon(0)=1 / 2$. For each $n \in \mathbb{N}$, we choose $\epsilon(n)>0$, so that $\epsilon(n)<\epsilon(n-1) / n$ and, for $i \leq n,\left|\log \left(a_{i}\right)\right|<|\log (\epsilon(n))|$, and $\left|b_{i}\right| \epsilon(n)<1$. These inequalities allow us to use (11.2.4) in the context of Proposition 12.2 .

Using Theorem 13.3, we find a sequence $(n(i))_{i \in \mathbb{N}}$ such that, for each $m \in \mathbb{N}$, the two sequences $\left(\mathcal{E}_{n(i)}\left(A_{m}\right)\right)_{i \in \mathbb{N}}$ and $\left(\mathcal{E}_{n(i)}\left(R_{m}\right)\right)_{i \in \mathbb{N}}$ each converge uniformly on compact subsets to quasiconformal homeomorphisms.

We fix the sequence $\sigma=(n(i))_{i \in \mathbb{N}}$ (actually, we will still need to replace $\sigma$ by a subsequence a few more times) and define the subgroup $G_{\sigma} \subset G_{b}$ to consist of all quasisymmetric $g$ such that the sequence $\left(\mathcal{E}_{n(i)}(g)\right)_{i \in \mathbb{N}}$ converges uniformly on compact subsets to an element of $Q \mathcal{C}$. By definition, $G_{\sigma}$ contains the groups $\left\{A_{m}\right\}$ and $\left\{R_{m}\right\}$. We can include in $G_{\sigma}$ any further countable collection of elements we desire in $G_{b}$, by going to a suitable subsequence. The limit gives us a homomorphism $\widehat{\mathcal{E}}: G_{\sigma} \rightarrow$ QC. We have $\widehat{\mathcal{E}}(g) \mid \mathbb{R}=g$.

Using (11.2.4), we see that, for any fixed $m,\left(d_{\mathcal{T}}\left(\Gamma_{n}\left(A_{m}\right), \mathrm{Id}\right)\right)_{n \in \mathbb{N}}$ converges to zero. Since translation does not affect Teichmüller distance in $\mathcal{Q S}$, we see that, for any fixed $m,\left(d_{\mathcal{T}}\left(\tau_{n}^{-1} \Gamma_{n}\left(A_{m}\right) \tau_{n}, \mathrm{Id}\right)\right)_{n \in \mathbb{N}}$ converges to 0 . We now apply Proposition 12.2 to show that there is a $k>1$, such that, for each $m$, the dilatation of $\widehat{\mathcal{E}}\left(A_{m}\right)$ is bounded by $k$.

According to Sullivan in [21] and Tukia in [23], we can find a single quasiconformal homeomorphism $q$ such that, for each $m, q \circ \widehat{\mathcal{E}}\left(A_{m}\right) \circ q^{-1}$ is a Möbius transformation. 
Without loss of generality, we may assume that $q$ fixes 0,1 and $\infty$. We write $r=q \mid \mathbb{R}$. For each $m, r A_{m} r^{-1}$ is a Möbius transformation fixing $\infty$, and is therefore an affine transformation. Moreover, if $A_{m}$ is a translation, then $r A_{m} r^{-1}$ is an affine transformation with no finite fixed points, so it is also a translation. For $i \in \mathbb{Z}$, let $B_{i}: \mathbb{R} \rightarrow \mathbb{R}$ be translation by $2^{-i}$. We prove by induction on $i$ that $r$ commutes with $B_{i}$ if $i \geq 0$.

To start the induction, we have shown that $r B_{0} r^{-1}$ is a translation sending 0 to 1 . Therefore $r B_{0} r^{-1}=B_{0}$. Suppose we know inductively that $r B_{i-1} r^{-1}=B_{i-1}$. Then $r B_{i} r^{-1}$ is a translation whose square is equal to $B_{i-1}$. Therefore $r B_{i} r^{-1}=B_{i}$ and the induction is complete.

From this it follows that $q$ fixes every point of the form $n / 2^{i}$, with $n \in \mathbb{Z}$ and $i \in \mathbb{N}$. Since such points are dense in $\mathbb{R}, q \mid \mathbb{R}=r$ is the identity map.

12.7 Definition As we warned immediately after Definition 12.6, we now change the definition of $\widehat{\varepsilon}$ by composing with conjugation by $q$.

For each $m, \widehat{\varepsilon}\left(A_{m}\right)$ is now a Möbius transformation whose restriction to $\mathbb{R}$ is equal to $A_{m}$. It follows that, for each $m, \widehat{\mathcal{E}}\left(A_{m}\right): \mathbb{U}^{2} \rightarrow \mathbb{U}^{2}$ is equal to the affine transformation $z \mapsto a_{m} z+b_{m}$. Also, for all $g \in G_{\sigma}$, it continues to be the case that $\widehat{\mathcal{E}}(g) \mid \mathbb{R}=g$.

In Paragraph 4.20, we showed how to associate subsets, like the $\ell_{r}$ of Section 4, to a 4-tuple of points in $\mathbb{S}^{1}$, with cross-ratio 2 . Given $x \in \mathbb{R}$ and $r>0$, the 4tuple $(x+r, \infty, x-r, x)$ has cross-ratio 2 . There is a corresponding conformal isomorphism between $f: S \rightarrow \mathbb{U}^{2}$, with $f(+\infty)=x, f(-\infty)=\infty, f(0)=x-r$ and $f(i \pi)=x+r$. The group $v\left(\mathbb{R}_{+}\right)$of Definition 4.1 is conjugated by $f$ to a group with support in the real interval $[x-r, x+r]$. The fixed points of this group are $x$, points of the interval $[x+r,+\infty]$ and points of the interval $[-\infty, x-r]$.

12.8 Specializing still further, we fix $x=-2$ and $r=1$, leading to a definite conformal isomorphism $f: S \rightarrow U$. Conjugation $c(f)$ by $f$ maps the group of quasisymmetric homeomorphisms of the boundary of $S$ to the group of quasisymmetric homeomorphisms of $\mathbb{R} \cup\{\infty\}$. Then $c(f) \circ v$ is a homomorphism from $\mathbb{R}_{+}$to the group of quasisymmetric homeomorphisms of $\mathbb{R}$ fixing $-1,-2$ and -3 , with support in $[-3,-1]$. We will pay particular attention to $c(f)(v(2))=(f \mid \mathbb{R}) \circ v(2) \circ(f \mid \mathbb{R})^{-1}$ which we will denote by $w: \mathbb{R} \rightarrow \mathbb{R}$. We replace $\sigma$ by a subsequence of $\sigma$, in such a way that $w \in G_{\sigma}$.

12.9 Proposition For each earthquake $R_{r}: \mathbb{R} \rightarrow \mathbb{R}$, such that $R_{r} \in G_{\sigma}, \widehat{\mathcal{E}}\left(R_{r}\right): \mathbb{U}^{2} \rightarrow$ $\mathbb{U}^{2}$ maps each ray in $\mathbb{U}^{2}$ through the origin to a ray through the origin. The restriction 
to each ray is a change of scale fixing 0 . Let $\lambda$ be as small as possible, with $0 \leq \lambda \leq \pi$ such that, for all $r>0$ and all $z \in \mathbb{U}^{2}$ such that $\lambda \leq \arg (z) \leq \pi$, we have $\widehat{\mathcal{E}}\left(R_{r}\right)(z)=z$. Then $\lambda<\pi$.

Proof Let $A_{a, b}$ be the affine map of $\mathbb{U}^{2} \cup \mathbb{R}$ defined by $A_{a, b}(z)=a z+b$. Consider the subgroup $H$ of hyperbolic transformations in $G_{\sigma}$ that fix 0 and $\infty$. Then $\widehat{\mathcal{E}}(H)$ is the "same" group of hyperbolic transformations acting on $\mathbb{U}^{2}$. We consider the orbit of any point $p \in \mathbb{U}^{2}$ under $\widehat{\mathcal{E}}(H)$. The orbit of $p$ is dense in the ray containing $p$. Since each earthquake $R_{r}$ commutes with each $A_{a, 0} \mid \mathbb{R}, \widehat{\mathcal{E}}\left(R_{r}\right)$ commutes with $\widehat{\mathcal{E}}\left(A_{a, 0} \mid \mathbb{R}\right)=A_{a, 0}$, whenever $R_{r}$ and $A_{a, 0}$ are both in $G_{\sigma}$. Since, for fixed $r$, $\widehat{\mathcal{E}}\left(R_{r}\right): \mathbb{U}^{2} \rightarrow \mathbb{U}^{2}$ is continuous, it must send each ray from the origin to a ray from the origin, and the restriction to any ray must be a change of scale, depending on the particular ray.

In order to complete the proof, we examine more closely the limiting process that produces $\widehat{\mathcal{E}}\left(R_{r}\right)$ and $\widehat{\mathcal{E}}(w)$, where $w$ is defined immediately before the statement of Proposition 12.9. The quasisymmetric homeomorphisms $R_{r}$ and $w$ have disjoint supports and therefore commute. Therefore $\tau_{n}^{-1} \circ \Gamma_{n}\left(R_{r}\right) \circ \tau_{n}$ commutes with $\tau_{n}^{-1} \circ$ $\Gamma_{n}(w) \circ \tau_{n}$. For $n>3, \Psi_{\epsilon(n)}$ is the identity on $[-3,-1]$, so that $\Gamma_{n}(w)=w$. This in turn implies that $\tau_{n}^{-1} \circ \Gamma_{n}\left(R_{r}\right) \circ \tau_{n}$ commutes with $\tau_{n}^{-1} \circ w \circ \tau_{n}$.

Let $f: S \rightarrow \mathbb{U}^{2}$ be the conformal isomorphism defined in Paragraph 12.8. Consider the homomorphism

$$
\begin{aligned}
v(r) & \mapsto f^{-1} q T_{n} \mathcal{E}\left(\tau_{n}^{-1}(f \mid \mathbb{R}) v(r)(f \mid \mathbb{R})^{-1} \tau_{n}\right) T_{n}^{-1} q^{-1} f \\
& =f^{-1} q \mathcal{E}_{n}\left((f \mid \mathbb{R}) v(r)(f \mid \mathbb{R})^{-1}\right) q^{-1} f .
\end{aligned}
$$

The justification for the equality in (12.9.a) is that $\Gamma_{n}$ is the identity on quasisymmetric maps of compact support, provided $n$ is large enough. Now (12.9.a) gives us a homomorphism $\mathcal{E}_{S, n}: v\left(\mathbb{R}_{+}\right) \rightarrow \mathcal{Q}(S)$, such that $\mathcal{E}_{S, n}(v(r)) \mid \partial S=v(r)$, which the only hypothesis necessary for the analysis in Section 4. We want to apply Lemma 4.21. The information we need is a bound for the dilatation of the homeomorphism of $\mathbb{U}^{2}$ in (12.9.a), when $r=2$.

We have $d_{\mathcal{T}}(v(2), \mathrm{Id}) \leq \log (2)$, as we see from the explicit extension of $v(2)$ to $S$. (In fact, the inequality is an equality, but we don't need this.) The argument of $\mathcal{E}$ in (12.9.a) is at the same Teichmüller distance from the identity. By Proposition 12.2, there is a $k_{3}>1$, independent of $n$, such that the dilatation of $\mathcal{E}\left(\left(\tau_{n}^{-1}(f \mid \mathbb{R}) v(r)(f \mid \mathbb{R})^{-1} \tau_{n}\right)\right.$ is at most $k_{3}$. To bound the dilatation of the homeomorphism of $\mathbb{U}^{2}$ in (12.9.a), we need only bring in the dilatation of $q$, once for $q^{-1}$ and once for $q$ itself. We find that the dilatation is bounded by some $k_{4}>1$, which is independent of $n$. This enables us 
to apply Lemma 4.21, with $K=k_{4}$, to obtain a fixed halfdisk $D$, independent of $n$, centred at -2 , such that, for each $n, D$ is contained in the set $f\left(\Omega_{+, n}\right)$ associated to $\mathcal{E}_{S, n}$ (see Definition 4.18). The situation is illustrated in Figure 12.9.i.

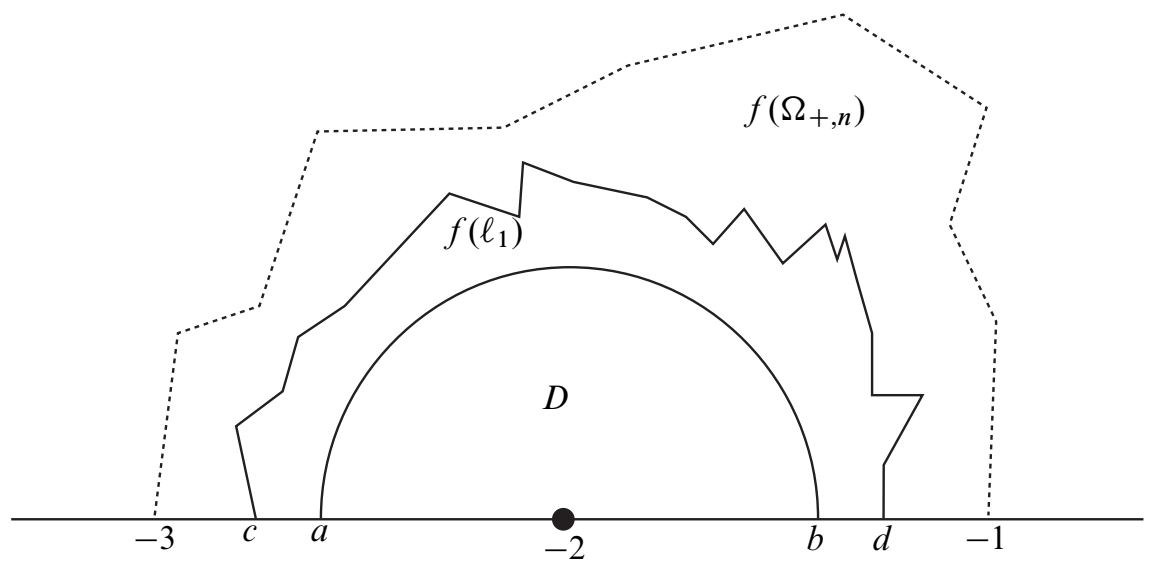

Figure 12.9.i: This picture illustrates the definition of $D$ towards the end of the proof of Proposition 12.9. The region bounded by the dotted line and part of the $x$-axis is $f\left(\Omega_{+, n}\right)$. We have $c=f(1)=-2-e^{-1}, d=$ $f(i \pi)=-2+e^{-1}, a=f\left(1+W_{0}\right)=-2-\exp \left(-1-W_{0}\right), b=f\left(1+W_{0}-\right.$ $i \pi)=-2+\exp \left(-1-W_{0}\right)$. One way to compute these coordinates is to use hyperbolic geometry and Lemma 4.21 .

By Theorem 5.1, $D$ is contained in the fixed point set of $q \mathcal{E}_{n}\left(R_{r}\right) q^{-1}$, for each $r>0$. If $R_{r} \in G_{\sigma}$, we can take the limit, with $n=n(i)$, as $i$ tends to infinity. We find that $\widehat{\mathcal{E}}\left(R_{r}\right)$ is the identity on $D$.

Since $\widehat{\mathcal{E}}\left(R_{r}\right)$ sends each ray through the origin to some ray through the origin by a change of scale, it must be fixed on any ray through the origin meeting $D$, as illustrated in Figure 12.9.ii

12.10 Note $R_{r}$ is an earthquake that fixes $(-\infty, 0)$ pointwise. We define the earthquake $L_{r}$ fixing $(0, \infty)$ by $L_{r} R_{r}=A_{r, 0} \mid \mathbb{R}$. A similar proof to that of Proposition 12.9 goes through for $L_{r}$ (or alternatively we can conjugate by reflection in the $y-$ axis), and we find the maximum $\rho$, such that $0<\rho \leq \pi$ and, such that, for any $r>0$ $\widehat{\mathcal{E}}\left(L_{r}\right)$ fixes each point $z \in \mathbb{U}^{2}$ such that $0<\arg (z) \leq \rho$. Since $L_{r} R_{r}=A_{r, 0} \mid \mathbb{R}$ and $\widehat{\mathcal{E}}\left(A_{r, 0} \mid \mathbb{R}\right)=A_{r, 0}$, we see that $0<\rho<\lambda<\pi$.

Our next task is to determine $\widehat{\mathcal{E}}\left(L_{r}\right)$ and $\widehat{\mathcal{E}}\left(R_{r}\right)$. Let $x \in \mathbb{R}$; then $A_{1, x}$ is translation by $x . A_{1, x} R_{r} A_{1,-x}$ is an earthquake based at $x$. This earthquake, denoted by $R_{x, r}$, 


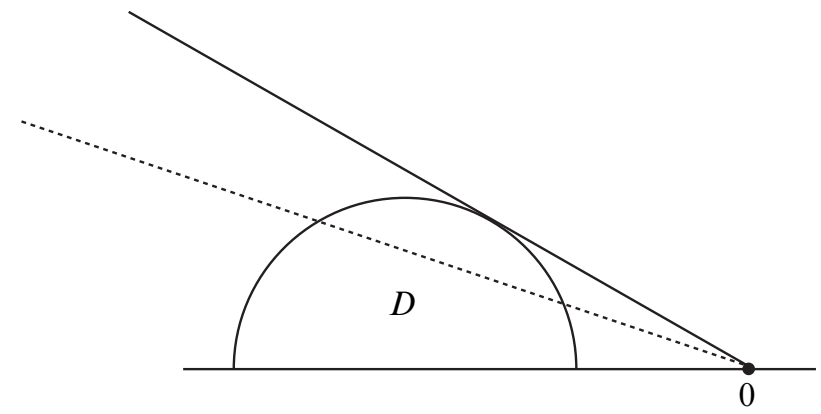

Figure 12.9.ii: This picture illustrates why $\widehat{\mathcal{E}}\left(R_{r}\right)$ is fixed on a wedge with one boundary edge along the negative $x$-axis. Each of the rays through 0 that meets $D$ (a typical ray is shown dotted) is pointwise fixed by $\widehat{\mathcal{E}}\left(R_{r}\right)$. This argument occurs right at the end of the proof of Proposition 12.9.

is the identity on $(-\infty, x)$. Similarly, we set $L_{x, r}=A_{1, x} L_{r} A_{1,-x}$, which is the identity on $(x, \infty)$.

12.11 Definition Let $0<\theta<\pi$. By a $\theta$-ray, we mean an infinite ray in $\mathbb{U}^{2}$ the upper halfplane, at an angle $\theta$ to the positive $x$-axis, and ending on $\partial \mathbb{U}^{2}$. We denote by $\operatorname{Ray}(\theta, x)$ the $\theta$-ray ending at $x \in \mathbb{R}$. In this section only, the open quadrant will mean the set of points $z \in \mathbb{U}^{2}$ with $\rho<\arg (z)<\lambda$, where $\lambda$ and $\rho$ are defined in Note 12.10. The closed quadrant is its closure in $\mathbb{C}$. Given a point $z$ in the quadrant, we give it coordinates $(u, v)$ as follows. Form a parallelogram with each side a segment in either a $\lambda$-ray or a $\rho$-ray, and with opposite vertices 0 and $z$. Then $u$ is the euclidean length of the $\rho$-segment and $v$ is the euclidean length of the $\lambda$-segment. See the illustration in Figure 12.12.i.

12.12 Theorem In the quadrant we have

$$
\widehat{\mathcal{E}}\left(L_{r}\right)(u, v)=(u, r v) \text { and } \widehat{\mathcal{E}}\left(R_{S}\right)(u, v)=(s u, v),
$$

provided that $L_{r}$ and $R_{s}$ are in $G_{\sigma}$.

Proof Since $L_{r}$ and $R_{S}$ commute with $A_{t, 0} \mid \mathbb{R}$, we see that $\widehat{\mathcal{E}}\left(L_{r}\right)$ and $\widehat{\mathcal{E}}\left(R_{S}\right)$ each permute the set of rays in $\mathbb{U}^{2}$ through the origin. Also, for each $t>0$ and $z \in \mathbb{U}^{2}$, we have $\widehat{\mathcal{E}}\left(L_{r}\right)(t z)=t \widehat{\mathcal{E}}\left(L_{r}\right)(z)$ and $\widehat{\mathcal{E}}\left(R_{S}\right)(t z)=t \widehat{\mathcal{E}}\left(R_{S}\right)(z)$. In particular, the set of fixed points for $\widehat{\varepsilon}\left(R_{S}\right)$ is a union of rays through 0 and similarly for $L_{r}$.

Let $x>0$. Then $R_{x, s}$ commutes with $L_{r}$. The fixed point set of the group

$$
\left\{\widehat{\mathcal{E}}\left(R_{x, s}\right)\right\}_{s>0}
$$




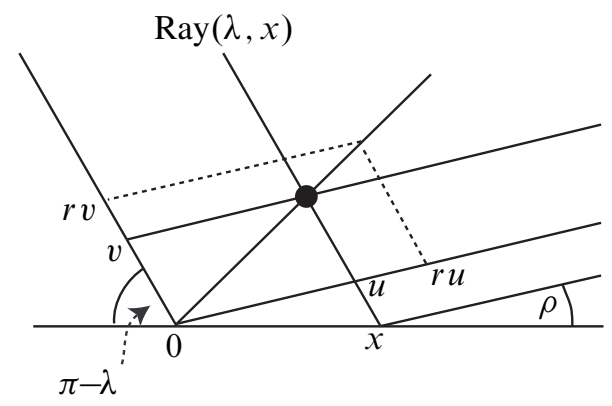

Figure 12.12.i: This picture illustrates the proof of Theorem 12.12.

has a component in $\mathbb{U}^{2}$ which abuts $\partial \mathbb{U}^{2}$ to the left of $x$ and it is the only such component. Therefore the component is preserved by $\widehat{\mathcal{E}}\left(L_{r}\right)$, and its boundary $\operatorname{Ray}(\lambda, x)$ is also preserved. See Figure 12.12.i. Interpreting this in the quadrant, we have $\widehat{\mathcal{E}}\left(L_{r}\right)(u, v)=\left(u, v^{\prime}\right)$ for some $v^{\prime}$. Since $\widehat{\mathcal{E}}\left(L_{r}\right)(z)=z$ if $\arg (z) \leq \rho$, we see that $v^{\prime}>0$. Similarly $\widehat{\mathcal{E}}\left(R_{S}\right)(u, v)=\left(u^{\prime}, v\right)$ for some $u^{\prime}>0$.

In order to determine $u^{\prime}$ and $v^{\prime}$ defined in the preceding paragraph, note that $A_{r, 0}=$ $\widehat{\mathcal{E}}\left(L_{r}\right) \widehat{\mathcal{E}}\left(R_{r}\right)$. Therefore

$$
(r u, r v)=A_{r, 0}(u, v)=\widehat{\mathcal{E}}\left(L_{r}\right)\left(u^{\prime}, v\right)=\left(u^{\prime}, v^{\prime \prime}\right) .
$$

So $u^{\prime}=r u$ and $\widehat{\mathcal{E}}\left(R_{r}\right)(u, v)=(r u, v)$. Similarly $\widehat{\mathcal{E}}\left(L_{r}\right)(u, v)=(u, r v)$.

In order to complete the proof of Theorem 12.1, we need to bring in the squaring map $\Sigma: \mathbb{R} \rightarrow \mathbb{R}$, defined by $\Sigma(t)=t^{2}$ if $t \geq 0$ and $\Sigma(t)=-t^{2}$ if $t \leq 0$. $\Sigma$ can be extended to the upper halfplane by $\Sigma\left(r e^{i \theta}\right)=r^{2} e^{i \theta}$, where the dilatation is 2 . So $\Sigma$ is quasisymmetric. Unfortunately, $\Sigma \notin G_{b}$. In fact, for each $n, \Sigma$ is not in the domain of $\Gamma_{n}$. So it is not at first clear how to extend $\widehat{\mathcal{E}}$ so that $\Sigma$ is contained in its domain.

We start with $P_{\epsilon(n)}$, defined in (11.2.6). Imitating, as far as possible, the construction of $\mathcal{E}_{n}$ in Equation (12.5.a), we set

$$
P_{n}=T_{n} \mathcal{E}\left(\tau_{n}^{-1} P_{\epsilon(n)} \tau_{n}\right) T_{n}^{-1} .
$$

12.13 Lemma Let $w$ be a word whose set of symbols consists of $\{\Sigma\} \cup X$, where $X$ is a finite subset of $G_{b}$. The word $w$ is a concatenation of these symbols and their formal inverses. We make the following two assumptions on $w$ :

(12.13.1) Evaluated as a composition of homeomorphisms, w: $\mathbb{R} \rightarrow \mathbb{R}$ is the identity. 
(12.13.2) The sum of exponents of $\Sigma$ in $w$ is zero.

Now substitute in $w$ by taking $P_{n}$ instead of $\Sigma$ and $\mathcal{E}_{n}(g)$ instead of $g \in X$, obtaining a quasiconformal homeomorphism $W_{n}: \mathbb{U}^{2} \cup \mathbb{R} \rightarrow \mathbb{U}^{2} \cup \mathbb{R}$. Then $W_{n}=$ Id.

Proof First substitute in $w$ by taking $P_{\epsilon(n)}$ instead of $\Sigma$ and $\Gamma_{n}(g)$ instead of $g \in X$, obtaining $w_{n}$. By Definition 12.3 and (11.2.6),

$$
w_{n} \mid\left(-M_{n}, M_{n}\right):\left(-M_{n}, M_{n}\right) \rightarrow\left(-M_{n}, M_{n}\right)
$$

is obtained from $w$ by conjugating with $\Psi_{\epsilon(n)}: \mathbb{R} \rightarrow\left(-M_{n}, M_{n}\right)$. Then (12.13.1) shows that this restriction of $w_{n}$ is the identity. On $\mathbb{R} \backslash\left(-M_{n}, M_{n}\right)$, the only terms in the word $w_{n}$ that are not the identity are those that come from $\Sigma$ by substituting $P_{\epsilon(n)}$. It follows from (12.13.2) that $w_{n}=$ Id here as well.

Conjugating the identity map by any homeomorphism gives the identity map and applying $\mathcal{E}$ to the identity map gives the identity map. It follows that $W_{n}=\mathrm{Id}$ as required.

Recall that we are using the sequence $\sigma=(n(i))_{i \in \mathbb{N}}$ to define $\widehat{\mathcal{E}}: G_{b} \rightarrow$ QC. Now suppose we knew, after passing to a subsequence of $\sigma$, that $\left(P_{n(i)}\right)_{i \in \mathbb{N}}$, as defined in Equation (12.12.a), converged uniformly on compact subsets to a quasiconformal $P: \mathbb{U}^{2} \cup \mathbb{R} \rightarrow \mathbb{U}^{2} \cup \mathbb{R}$. Lemma 12.13 would then show that we can extend the domain of $\widehat{\varepsilon}$ to the group generated by $G_{b}$ and $\Sigma$, by defining $\widehat{\mathcal{E}}(\Sigma)=P$. (This statement assumes that (12.13.2) follows from (12.13.1). This is probably true, though we want to avoid taking the time and trouble to prove it. To obtain a rigorous treatment, the statement should be interpreted as providing motivation, rather than being part of a formal proof.) However, the maximal dilatations of the $P_{n(i)}$ are not uniformly bounded, so we cannot use the usual route for proving convergence.

Instead we proceed as follows. Recall the definition of $L_{n}$, given just after the proof of Proposition 12.2. The conjugating map $\Psi_{\epsilon(n)}: \mathbb{R} \rightarrow\left(-M_{n}, M_{n}\right)$ is the identity on $\left[-L_{n}, L_{n}\right]$. We define $S_{n}: \mathbb{U}^{2} \rightarrow \mathbb{U}^{2}$ as follows.

$$
S_{n}\left(r e^{i \theta}\right)= \begin{cases}r^{2} e^{i \theta} & \text { if } r \leq L_{n}^{1 / 3} \\ \sqrt{r L_{n}} e^{i \theta} & \text { if } L_{n}^{1 / 3} \leq r \leq L_{n} \\ r e^{i \theta} & \text { if } L_{n} \leq r\end{cases}
$$

The dilatation of $S_{n}$ is 2: the important point is that it is independent of $n$. We set $s_{n}=S_{n} \mid \mathbb{R}$ and then $d_{\mathcal{T}}\left(s_{n}, \mathrm{Id}\right) \leq \log (2)$. Also, $s_{n}$ is supported on $\left[-L_{n}, L_{n}\right]$ and so 
$\tau_{n}^{-1} s_{n} \tau_{n}$ keeps 0,1 and $\infty$ fixed. In addition, $\Gamma_{n}\left(s_{n}\right)=s_{n}$. It follows from Proposition 12.2 that there is a $k_{5}>1$, such that, for each $n$, the dilatation of

$$
Q_{n}=q T_{n} \mathcal{E}\left(\tau_{n}^{-1} \Gamma_{n}\left(s_{n}\right) \tau_{n}\right) T_{n}^{-1} q^{-1}=q \mathcal{E}_{n}\left(s_{n}\right) q^{-1}
$$

is bounded by $k_{5}$. Taking a subsequence of $\sigma=(n(i))_{i \in \mathbb{N}}$, we may assume, by Theorem 13.3 that this converges uniformly on compact subsets of $\mathbb{U}^{2} \cup \mathbb{R}$ to a quasiconformal limit, which we denote by $S: \mathbb{U}^{2} \cup \mathbb{R} \rightarrow \mathbb{U}^{2} \cup \mathbb{R}$. We have $S(x)=x^{2}$ for $x \geq 0$ and $S(x)=-x^{2}$ for $x \leq 0$.

Now we compare $Q_{n}$ with $q P_{n} q^{-1}$, where $P_{n}$ is defined in Equation (12.12.a). Since $q \mid \mathbb{R}$ is the identity, $q P_{n} q^{-1}$ and $Q_{n}$ agree on the interval $I_{n}=\left(-L_{n}^{1 / 3}, L_{n}^{1 / 3}\right)$. As in Paragraph 4.20, the 4-tuple $\left(-L_{n}^{1 / 3}, 0, L_{n}^{1 / 3}, \infty\right)$ determines a conformal conjugacy with the infinite strip $S$. From Theorem 5.1 we have an open neighbourhood $\Omega_{n}$ of $I_{n}$ in $\overline{\mathbb{U}^{2}}$, with the property that, if a quasisymmetric map $f$ is the identity on $I_{n}$, then $\mathcal{E}(f)$ is the identity on $\Omega_{n}$. Using Lemma 4.21, we find a closed halfdisk $D_{n} \subset \Omega_{n}$ centred at 0 . The hyperbolic distance of $D_{n}$ to the geodesic joining the endpoints of $I_{n}$ can be kept fixed independently of $n$, as shown in (4.16.1). It follows that $D_{n}$ expands with $n$ and that $\bigcup_{n} D_{n}=\mathbb{U}^{2} \cup \mathbb{R}$.

For each $n, q P_{n} q^{-1}\left|I_{n}=Q_{n}\right| I_{n}$ is the map $x \mapsto \pm x^{2}$. Hence $q P_{n} q^{-1} \mid D_{n}=$ $Q_{n} \mid D_{n}$. Given a compact subset $C \subset \mathbb{U}^{2} \cup \mathbb{R}, C \subset D_{n}$ for some $n$. It follows that $\left(q P_{n(i)} q^{-1}\right)_{i \in \mathbb{N}}$ converges to the quasiconformal homeomorphism $S$ uniformly on $C$, and therefore uniformly on each compact subset.

12.14 Lemma We make the same assumptions about $w$ that are made in Lemma 12.13. Substitute in $w$ by taking $S$ instead of $\Sigma$ and $\widehat{\mathcal{E}}(g)$ instead of $g \in X$, obtaining a quasiconformal homeomorphism $W: \mathbb{U}^{2} \cup \mathbb{R} \rightarrow \mathbb{U}^{2} \cup \mathbb{R}$. Then $W=\mathrm{Id}$.

Proof Let $\sigma=(n(i))_{i \in \mathbb{N}}$. We apply Lemma 12.13 with $n=n(i)$. Replacing $P_{n}$ by $q P_{n} q^{-1}$ and $\mathcal{E}_{n}(g)$ by $q \mathcal{E}_{n}(g) q^{-1}$, we get another version of Lemma 12.13. Letting $i$ tend to infinity, we obtain the statement we want.

Now consider the word $w=S \widehat{\mathcal{E}}\left(L_{r}\right) S^{-1} \widehat{\mathcal{E}}\left(L_{1 / r}\right) \widehat{\mathcal{E}}\left(L_{1 / r}\right)$. We see that $w$ is the identity as a map $\mathbb{R} \rightarrow \mathbb{R}$. Also the sum of exponents of $S$ is 0 . It follows from Lemma 12.14 that $w=\mathrm{Id}$ as a map $\mathbb{U}^{2} \rightarrow \mathbb{U}^{2}$. So $S \widehat{\mathcal{E}}\left(L_{r}\right)=\widehat{\mathcal{E}}\left(L_{r}\right) \widehat{\mathcal{E}}\left(L_{r}\right) S$. Similarly $S \widehat{\mathcal{E}}\left(R_{S}\right)=\widehat{\mathcal{E}}\left(R_{S}\right) \widehat{\mathcal{E}}\left(R_{S}\right) S$.

12.15 Lemma $S$ preserves the rays $\operatorname{Ray}(\lambda, 0)$ and $\operatorname{Ray}(\rho, 0)$ (see Definition 12.11). $S$ preserves the quadrant between these two rays. 
Proof We know from Theorem 12.12 that $\widehat{\mathcal{E}}\left(R_{r}\right)$ fixes no point of the open quadrant, unless $r=1$, and similarly for $\widehat{\mathcal{E}}\left(L_{S}\right)$. Using the fact that $\widehat{\mathcal{E}}\left(R_{r}\right) \widehat{\mathcal{E}}\left(L_{r}\right)=A_{r, 0}$ and the fact that $\widehat{\mathcal{E}}\left(L_{r}\right)$ fixes any point with argument between 0 and $\rho$, we see that $\widehat{\mathcal{E}}\left(R_{r}\right)$ fixes no point in the sector between 0 and $\rho$, unless $r=1$. So the fixed point set of $\widehat{\mathcal{E}}\left(R_{r}\right)$ with $r \neq 1$ is exactly the sector of points with argument between $\lambda$ and $\pi$.

The equation $S \widehat{\mathcal{E}}\left(R_{r}\right)=\widehat{\mathcal{E}}\left(R_{r^{2}}\right) S$ shows that, if $z$ is in the sector $\lambda \leq \arg (z) \leq \pi$, then $S(z)$ is a fixed point for each $\widehat{\mathcal{E}}\left(R_{t}\right)$ with $t=r^{2}$. But this means that $S(z)$ is in the same sector. This proves the lemma.

Using Theorem 12.12, we now work out the value of $S$ in the quadrant of Definition 12.11. We write $S(u, v)=\left(s_{1}(u, v), s_{2}(u, v)\right)$. The equation $S \widehat{\mathcal{E}}\left(L_{r}\right)=\widehat{\mathcal{E}}\left(L_{r^{2}}\right) S$ implies that $s_{1}(u, r v)=s_{1}(u, v)$ and $s_{2}(u, r v)=r^{2} s_{2}(u, v)$. Therefore $s_{1}(u, v)$ is independent of $v$ and can be written as $s_{1}(u)$. Similarly $s_{2}(u, v)$ can be written $s_{2}(v)$. We have $s_{2}(r v)=r^{2} s_{2}(v)$ and $s_{1}(r u)=r^{2} s_{1}(u)$.

It follows that there are constants $a>0$ and $b>0$, such that $S(u, v)=\left(a u^{2}, b v^{2}\right)$. The coordinates $(u, v)$ in the quadrant are affinely related to conformal coordinates. So we see that $S$ is not quasiconformal on the quadrant. This contradiction completes the proof of Theorem 12.1 and therefore the proof of Theorem 1.1.

\section{Standard results}

Most of the statements found in this section are proved in the book [1] by Ahlfors.

13.1 Theorem Let $f: \mathbb{D}^{2} \rightarrow \mathbb{D}^{2}$ be a quasiconformal homeomorphism. Then $f$ can be extended to a continuous quasiconformal homeomorphism of the closed disk.

This is a consequence of Mori's Theorem—see [1, page 47].

There is a stronger form of this, also a consequence of Mori's Theorem.

13.2 Theorem Given a family of uniformly quasiconformal homeomorphisms of the open unit disk (that is, their maximal dilatations are uniformly bounded) and a number $r$, such that $0 \leq r<1$, and such that, for each $u$ in the family, the absolute value $|u(0)|<r$, then the family is equicontinuous. (This means that, given $\epsilon>0$, there exists $\delta>0 \ldots$, that works for every $u$ in the family.

13.3 Theorem Let $U$ be a connected open subset of $\mathbb{S}^{2}$, and let $\left(f_{n}\right)_{n \in \mathbb{N}}$ be a sequence of uniformly quasiconformal homeomorphisms of $U$ with itself. Then 
either some subsequence converges to a point, or some subsequence converges to a quasiconformal homeomorphism of $U$. The convergence is uniform on any fixed compact subset of $U$. If $U=\mathbb{D}^{2}$, then each of these functions can be continuously extended to $\overline{\mathbb{D}^{2}}$, and then uniform convergence to a quasiconformal homeomorphism can be replaced by uniform convergence on $\mathbb{D}^{2}$.

This is proved at [1, page 51, Theorem 2].

13.4 The $M$-condition In [2], Beurling and Ahlfors gave the $M$-condition for an orientation preserving homeomorphism of $\mathbb{R}$ to be quasisymmetric. This is discussed in [1, Chapter IV]. The condition says that an orientation preserving homeomorphism $h$ of $\mathbb{R}$ is quasisymmetric (the restriction of a quasiconformal homeomorphism on the closed unit disk keeping one point of $\mathbb{S}^{1}$ fixed), if and only if there exists $M \geq 1$ such that, for all $x \in \mathbb{R}$ and for all $t>0$,

$$
M^{-1} \leq \frac{h(x+t)-h(x)}{h(x)-h(x-t)} \leq M .
$$

In fact, if $M$ is chosen as small as possible for a fixed $h$, then using $\log (M)$ is more or less equivalent to using the Teichmüller metric.

13.5 Beltrami differentials A quasiconformal homeomorphism $\phi: \mathbb{D}^{2} \rightarrow \mathbb{D}^{2}$ is differentiable almost everywhere. The Beltrami differential is defined by

$$
\mu(z)=\frac{\partial \phi / \partial \bar{z}}{\partial \phi / \partial z} .
$$

In some ways it is natural to regard $\mu$ as an almost everywhere defined function into the hyperbolic plane, thought of as the unit disk provided with the Poincaré metric. This is pointed out in [1, page 10]. In [1, page 99], Beltrami differentials are used to prove that any quasiconformal homeomorphism is a product of quasiconformal homeomorphisms that are near the identity, in fact simultaneously near the identity, in terms both of the Teichmüller metric and of the uniform metric.

We frequently use a result proved by the authors, working with Al Marden, namely [6, Theorem 5.1]). To state this result, recall that the unit disk with the Poincaré metric is a model for the hyperbolic plane.

13.6 Theorem There is an explicit function $\epsilon:[1, \infty) \rightarrow[0, \infty)$, with the following property. Let $f: \mathbb{D}^{2} \rightarrow \mathbb{D}^{2}$ be a $K$-quasiconformal homeomorphism. Then $f$ is a $(K, \epsilon(K))$-quasi-isometry with respect to the hyperbolic metric. 
We also include here the following topological lemma.

13.7 Lemma Let $X$ be a connected compact Hausdorff space, and let $A$ and $B$ be disjoint non-empty closed connected subsets of $X$. Let $C$ be the component of $X \backslash A$ containing $B$. Then $\bar{C} \cap A \neq \varnothing$.

Proof It is sufficient to show that, for each open neighbourhood $U$ of $A, \bar{C} \cap \bar{U} \neq \varnothing$. By contradiction, we assume that $\bar{C} \cap \bar{U}=\varnothing$. Let $D$ be the component of $X \backslash U$ containing $B$. We know that $D \subset C \subset \bar{C}$, and so $D \cap \bar{U}=\varnothing$.

It is standard (but not trivial) that each component of a compact Hausdorff space is the intersection of subsets that are simultaneously open and closed. We apply this to the compact Hausdorff space $X \backslash U$ and the component $D$. Let $E$ be a set that is open and closed in $X \backslash U$, with $D \subset E$ and $E \cap \bar{U}=\varnothing$. It follows that $E$ is both open closed in $X$. Since $X$ is connected, $E=\varnothing$ or $E=X$. But each of these is impossible.

There is an example due to Knaster and Kuratowski (see [15]) of a connected subset $X$ of the plane and a point $x \in X$, such that $X \backslash\{x\}$ is non-empty and each of its components is a point.

\section{References}

[1] L V Ahlfors, Lectures on quasiconformal mappings, Manuscript prepared with the assistance of C J Earle, Jr., Van Nostrand Mathematical Studies 10 (1966) MR0200442

[2] A Beurling, L Ahlfors, The boundary correspondence under quasiconformal mappings, Acta Math. 96 (1956) 125-142 MR0086869

[3] A Casson, D Jungreis, Convergence groups and Seifert fibered 3-manifolds, Invent. Math. 118 (1994) 441-456 MR1296353

[4] A Douady, C J Earle, Conformally natural extension of homeomorphisms of the circle, Acta Math. 157 (1986) 23-48 MR857678

[5] D B A Epstein, Functors between tensored categories, Invent. Math. 1 (1966) 221-228 MR0213412

[6] D B A Epstein, A Marden, V Markovic, Quasiconformal homeomorphisms and the convex hull boundary, Ann. of Math. (2) 159 (2004) 305-336 MR2052356

[7] D B A Epstein, W P Thurston, Transformation groups and natural bundles, Proc. London Math. Soc. (3) 38 (1979) 219-236 MR531161

[8] D Gabai, Convergence groups are Fuchsian groups, Ann. of Math. (2) 136 (1992) 447-510 MR1189862 
[9] F P Gardiner, D P Sullivan, Symmetric structures on a closed curve, Amer. J. Math. 114 (1992) 683-736 MR1175689

[10] É Ghys, Prolongements des difféomorphismes de la sphère, Enseign. Math. (2) 37 (1991) 45-59 MR1115743

[11] J A Giblin, On the structure of homeomorphism groups, $\mathrm{PhD}$ thesis, University of Warwick (2007)

[12] A Hinkkanen, Abelian and nondiscrete convergence groups on the circle, Trans. Amer. Math. Soc. 318 (1990) 87-121 MR1000145

[13] A Hinkkanen, The structure of certain quasisymmetric groups, Mem. Amer. Math. Soc. 83 (1990) MR948926

[14] S P Kerckhoff, The Nielsen realization problem, Ann. of Math. (2) 117 (1983) 235-265 MR690845

[15] B Knaster, C Kuratowski, Sur les ensembles connexes, Fund. Math. 2 (1922) 206-255

[16] V Markovic, Realization of the mapping class group by homeomorphisms To be published in Invent. Math. (2007) Available at http://www.maths.warwick.ac.uk/ $\sim$ markovic

[17] V Markovic, Quasisymmetric groups, J. Amer. Math. Soc. 19 (2006) 673-715 MR2220103

[18] J N Mather, Integrability in codimension 1, Comment. Math. Helv. 48 (1973) 195-233 MR0356085

[19] S Morita, Geometry of characteristic classes, Translations of Mathematical Monographs 199, Amer. Math. Soc. (2001) MR1826571 Translated from the 1999 Japanese original, Iwanami Series in Modern Mathematics

[20] J F Plante, Subgroups of continuous groups acting differentiably on the half-line, Ann. Inst. Fourier (Grenoble) 34 (1984) 47-56 MR743621

[21] D Sullivan, On the ergodic theory at infinity of an arbitrary discrete group of hyperbolic motions, from: "Riemann surfaces and related topics: Proceedings of the 1978 Stony Brook Conference (Stony Brook, 1978)", Ann. of Math. Stud. 97, Princeton Univ. Press (1981) 465-496 MR624833

[22] T Tsuboi, On the foliated products of class $C^{1}$, Ann. of Math. (2) 130 (1989) 227-271 MR1014925

[23] P Tukia, On two-dimensional quasiconformal groups, Ann. Acad. Sci. Fenn. Ser. A I Math. 5 (1980) 73-78 MR595178

[24] P Tukia, Homeomorphic conjugates of Fuchsian groups, J. Reine Angew. Math. 391 (1988) 1-54 MR961162 
Mathematics Institute, University of Warwick, Coventry CV4 7AL, UK

Department of Mathematics, Universiy of Stony Brook, Stony Brook, NY 11794, USA

David.Epstein@warwick.ac.uk, markovic@math.sunysb.edu

Proposed: Martin Bridson

Seconded: Jean-Pierre Otal, Wolfgang Lück
Received: 28 September 2006

Accepted: 14 February 2007 